\title{
Superconducting Submillimeter and Millimeter Wave Detectors
}

by

Michael Nahum
LBL -33039

DE93 007656

B.S. (University of Arizona) 1987

A dissertation submitted in partial satisfaction of the requirements for the degree of

Doctor of Philosophy

in

Physics

in the

GRADUATE DIVISION

of the

UNIVERSITY of CALIFORNIA at BERKELEY

* This work was supported in part by the Director, Office of Energy

Research, Office of Basic Energy Sciences, Materials Sciences Division of the

U. S. Departument of Energy under Contract No. DE-AC03-76-SF00098.

October 20,1992 


\title{
Superconducting Submillimeter and Millimeter Wave Detectors
}

\author{
Copyright $\odot 1992$
}

by

Michael Nahum

The U.S. Department of Energy has the right to use this thesis for any purpose whatsoever including the right to reproduce all or any part thereof 
Abstract

Superconducting Submillimeter and Millimeter Wave Detectors

by

Michael Nahum

Doctor of Philosophy in Physics

University of California at Berkeley

Professor P. L. Richards, Chair

The series of projects described in this dissertation was stimulated by the discovery of high temperature superconductivity. Our goal was to develop useful applications which would be competitive with the current state of technology. The high- $T_{C}$ microbolometer was developed into the most sensitive direct detector of millimeter waves, when operated at liquid nitrogen temperatures. The thermal boundary resistance of thin $\mathrm{YBa}_{2} \mathrm{Cu}_{3} \mathrm{O}_{7-\delta}$ films was subsequently measured and provided direct evidence for the bolometric response of high- $T_{c}$ films to fast (ns) laser pulses. The low $-T_{c}$ microbolometer was developed and used to make the first direct measurements of the frequency dependent optical efficiency of planar lithographed antennas. The hot-electron microbolometer was invented less than a year prior to the writing of this dissertation. Our analysis, presented here, indicates that it should be possible to attain up to two orders of magnitude higher sensitivity than that of the best available direct detectors when operated at the same temperature. The temperature readout scheme for this device could also be used to measure the intrinsic interaction between electrons and phonons in a metal with a sensitivity that is five orders of magnitude better than in previous measurements. Prelinainary measurements of quasiparticle trapping effects at the interface between a metal and a superconductor are also presented. 


\section{CONTENTS}

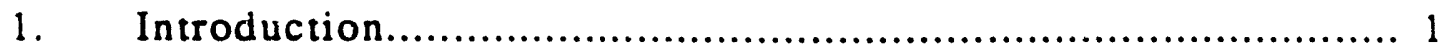

2. Bolometer Theory.............................................................. 4

2.1 Introduction....................................................... 4

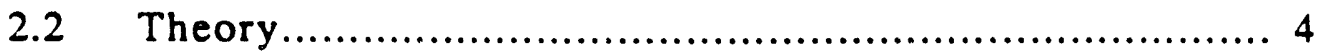

2.3 Measurement of Bolometer Responsivity............................... 7

2.4 Noise in Bolometers................................................ 8

2.5 Antenna Coupled Bolometers............................................ 12

2.6 Antenna Theory........................................................ 13

3. High- $T_{c}$ Microbolometer...................................................... 19

3.1 Introduction....................................................... 19

3.2 Energy Dissipation................................................ 20

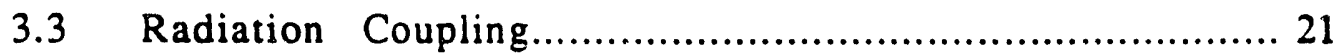

3.4 Device Fabrication.................................................... 23

3.5 Electrical and Optical Characterization...................................... 25

3.6 Measurements of the Optical Efficiency................................. 32

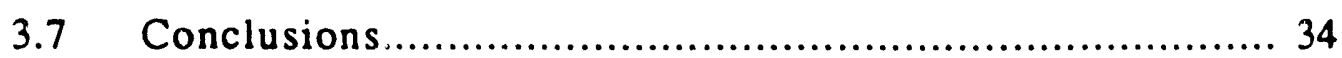

4. Thermal Boundary Resistance for YBCO Films.................................... 35

4.1 Introduction......................................................... 35

4.2 Theoretical Background................................................ 37

4.3 Measurement Scherne.................................................. 40

4.4 Sample Fabrication................................................ 43

4.5 Experimental Results................................................... 44

4.6 Conclusions...................................................... 48 


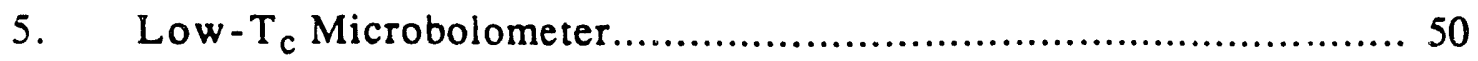

5.1 Introduction.................................................... 50

5.2 Operating Principles.................................................... 51

5.3 Experimental Apparatus and Device Fabrication........................... 56

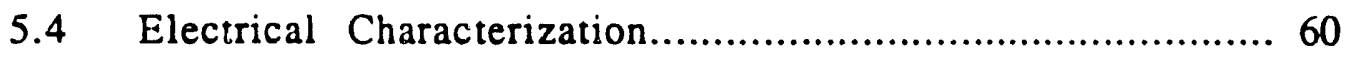

5.5 Frequency Dependent Optical Efficiency of Log-Periodic Antennas... 65

5.6 Design of Tuning Elements and Narrow Bandpass Filters.............. 79

5.7 Conclusions........................................................ 84

6. Design Analysis of a Hot-Electron Microbolometer................................ 85

6.1 Introduction..................................................... 85

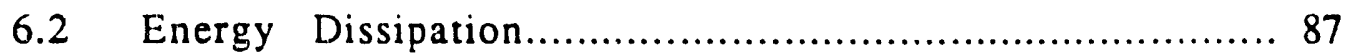

6.3 Temperature Readout............................................... 89

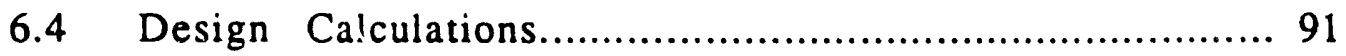

6.5 Tunneling as a Probe of Hot Electrons................................... 95

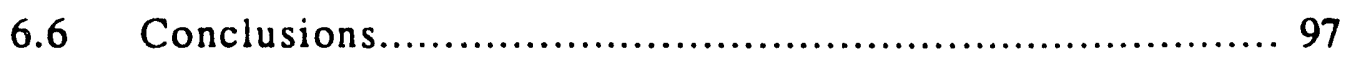

7. Proposed Measurements of Quasiparticle Trapping Effects...................... 98

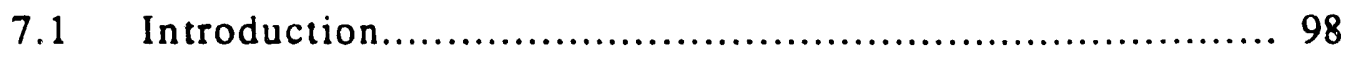

7.2 Theoretical Background.................................................. 99

7.3 Measurement Scheme............................................... 103

7.4 Preliminary Results and Conclusions................................... 109

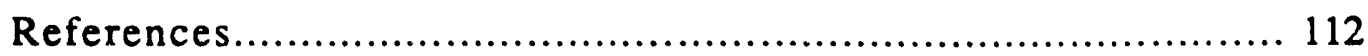

Appendix A: Transformers as Amplifiers for Low Impedance Devices........ 120 


\section{ACKNOWLEDGMENTS}

It is my pleasure to acknowledge all the people that made this dissertation possible. First and foremost, I would like to thank my advisor Professor Paul Richards. His patience, guidance, and persistence helped train an inexperienced undergraduate into a professional member of the physics community.

The past four years of my tenure here at the Richards Group were truly enjoyable (except of course, for those days when nothing seemed to work). The people who deserve special credit for developing and maintaining such a 'friendly' atmosphere are: John Birmingham, for his periodic verbal abuse, and for finally managing to out-lift me in the weight room. Andre ('flight bolometer') Clapp for teaching me how to claim what is rightfully, or un-rightfully, yours. Warren Holmes, for offering to split whatever food he had. Carl Mears, through his skepticism, and his "Hummmmph" and "buffoonery!!" statements, for teaching me to think things through prior to making wild speculations. David Miller, for trusting me with his tool box, and Simon Verghese, for his everlasting willingness to talk about, well, anything (but mostly physics).

My wife Beth has been a wonderful companion during the past two years. Her patience, and encouragement have been an indispensable source of energy and motivation. I thank Beth for teaching me how to ski, especially for helping me 'get organized' after colliding with various stationary and moving objects (including herself).

Finally, I would like to thank my parents for their constant support throughout the years, and for giving up so much for my education. I thank my mother for teaching me to enjoy and appreciate the small things in life, and for teaching me how to cook. I thank my father for teaching me to be persistent and stubborn, and also pragmatic and flexible. I also thank him for those countless hours, showing me the simplicity of physics, when it all seemed so confusing. 


\section{Chapter l:-Introduction}

The series of projects described in this dissertation was stimulated by the discovery of high temperature superconductivity. ${ }^{1}$ Our initial goal was to develop useful applications which would be compatible with the current state of technology. Two immediate applications that came to mind were high temperature Superconducting Quantum Interference Devices (SQUIDs), ${ }^{2}$ and high- $\mathrm{T}_{\mathrm{c}}$ transition-edge bolometers. ${ }^{3}$ Having just joined a group whose expertise is in developing and using some of the most sensitive detectors of infrared and millimeter waves, I naturally chose to pursue the latter option. The goal in the development of high- $T_{c}$ bolometers was not to outperform their low temperature counterparts, but rather to bridge the gap between these highly specialized detectors, and lower sensitivity detectors operated at room temperature. In 1989 Q. Hu and P. L. Richards proposed the antenna-coupled high- $\mathrm{T}_{\mathrm{c}}$ microbolometer as a detector of submillimeter and millimeter waves. ${ }^{4}$ They estimated that the sensitivity of this detector could exceed that of detectors operated at room temperature by approximately two orders of magnitude. Furthermore, Conductus Inc. became interested in the commercial aspects of high- $T_{c}$ infrared detectors and had agreed to supply us with high quality films of $\mathrm{YBa}_{2} \mathrm{Cu}_{3} \mathrm{O}_{7-\delta}$ (YBCO). Thus, one of my first projects was to learn how to fabricate the high- $\mathrm{T}_{\mathrm{c}}$ microbolometer, and to measure and optimize its performance. ${ }^{5}$

During the same period, many groups began measuring the response of thin $\mathrm{YBCO}$ films to pulsed laser radiation. A very high speed of response, on the order of several nanoseconds, was commonly observed. The consensus among the community at that time was that simple heating effects could not possibly explain this response time because thermal models predicted response times on the order of tens of microseconds for heat transfer through the underlying substrate, not nanoseconds. As a result, a variety of novel, non-bolometric mechanisms were proposed to explain these observations. ${ }^{6-9}$ During several discussions with Professor P. L. Richards, the question arose whether the thermal 
boundary resistance between the YBCO film and the underlying substrate could possibly account for many of the experimental observations. At first this did not seem likely since it was well known that thermal boundary resistances, which scale as $\mathrm{T}^{-3}$, should be negligible at these high temperatures. It quickly became clear to us however, that this may not be the case in experiments involving fast laser pulses incident on thin films. We thus embarked on a set of experiments to measure directly the magnitude of the thermal boundary resistance between YBCO films and the underlying substrate. ${ }^{10}$

Having gained a solid understanding of antenna-coupled bolometers, and of thermal boundary resistances, we then inquired whether a low- $T_{c}$ microbolometer could compete with conventional composite bolometers operated at and below $4.2 \mathrm{~K}$. We came to the conclusion that this approach could not only lead to an order of magnitude improvement in sensitivity, but also that several of the obstacles associated with the development of array systems could be overcome. ${ }^{11,12}$ Needless to say, we decided to pursue this approach. We established a collaborative effort with Professor D. E. Prober's group at Yale University in which the devices would be fabricated at Yale whereas all of the measurements would be carried out at Berkeley.

We invented the hot-electron microbolometer a little less than a year prior to the writing of this dissertation. Our initial estimates suggested that it could be possible to attain approximately two orders of magnitude higher sensitivity than that of the best available bolometers when operated at the same temperature. ${ }^{13}$ The hot-electron microbolometer would thus be the most sensitive direct detector of submillimeter and millimeter waves, and could potentially be the detector of choice in many applications which require very high sensitivities and array compatibility. In addition, the readout scheme that we invented could be used to measure the intrinsic interaction between electron and phonons in a metal at low temperatures, with a much higher resolution than the conventional SQUID readout method. ${ }^{14}$ Although these concepts have not been tested, I have decided to include the analysis in this dissertation. 
The common theme for the low temperature detector concepts is the need to trap energy in a very small active region, while making electrical contact to that region. At first, these requirements seem to be mutually exclusive, since metals are known to be very good conductors of heat. If however, the contacts are made of a superconductor whose $T_{c}$ is much higher than the operating temperature, then the Andreev reflection of quasiparticles at that interface insures that the energy remains trapped in the active region. ${ }^{15} \mathrm{My}$ last few months at Berkeley were focused towards designing an experiment to make direct measurements of the trapping of thermal energy at the interface between a metal and a superconductor as a function of the energy gap of the superconducting interface. Although these measurements have not been completed, I have decided to include a short progress report.

The organization of this dissertation will follow the order in which the experiments were carried out. I will first start with a general overview of bolometers and will discuss in some detail, the various sources of noise which can limit their sensitivity. The advantages of reducing the size of the active region will lead to a discussion of antenna coupled microbolometers. I will then proceed by describing the experiments on the high$\mathrm{T}_{\mathrm{c}}$ microbolometer, and will conclude the discussion of the high- $\mathrm{T}_{\mathrm{c}}$ work by describing our measurements of the thermal boundary resistance of YBCO films. The motivation for our work on the low temperature microbolometers will be introduced through a brief discussion of the applications which would benefit from such a device. Measurements of the properties of these novel devices will be discussed as well as the physical phenomena which are pertinent to their behavior. I will conclude by describing some very preliminary experiments on the quasiparticle trapping effects at the interface between a metal and a superconductor. 


\section{Chapter 2: Principles of Antenna Coupled Bolometers}

\subsection{Introduction}

A bolometer is a thermal detector which in principle, can detect radiation from $\mathrm{dc}$ to $\gamma$ rays. Bolometers are the most sensitive direct detectors of radiation for frequencies between $3 \mathrm{GHz}$ to $1 \mathrm{THz}$, and have also been used extensively up to $10 \mathrm{THz}$. All bolometers include an absorbing element which converts the incident radiation to heat, and a thermal link between the active region and the heat sink. The rise in temperature of the thermally active region is measured by a resistive thermometer. Bolometric detectors for infrared and millimeter waves are used extensively in laboratory and astronomical applications. Bolometers that operate between LHe temperature and room temperature are mainly used for laboratory far infrared spectroscopy, while bolometers operating between $0.02 \mathrm{~K}$ and $4.2 \mathrm{~K}$ are used for very sensitive ground and space-based astronomical and astrophysics observations. The design parameters of the bolometer depend on the particular application, and in most cases, the optimization of the bolometer performance involves a complicated tradeoff between sensitivity, speed of response, dynamic range, etc. In what follows, I will give an overview of the basic operating principles of bolometers, and will then motivate the concept of antenna coupled bolometers (also referred to as microbolometers). I will conclude this chapter with a discussion of the properties of planar antennas.

\subsection{Bolometer Theory}

As previously mentioned, a bolometer consists of a thermally active region of heat capacity $C$ coupled to a temperature bath through a thermal conductance $G$ (Fig. 2.1). The thermally active region includes an absorber and a thermometer. The temperature rise $\Delta \mathrm{T}=\mathrm{T}_{0}+\mathrm{T}_{1} \exp (\mathrm{i} \omega \mathrm{t})$ of the active region due to a modulated radiant power input $P=P_{0}+P_{1} \exp (i \omega t)$ is found from simple energy considerations 


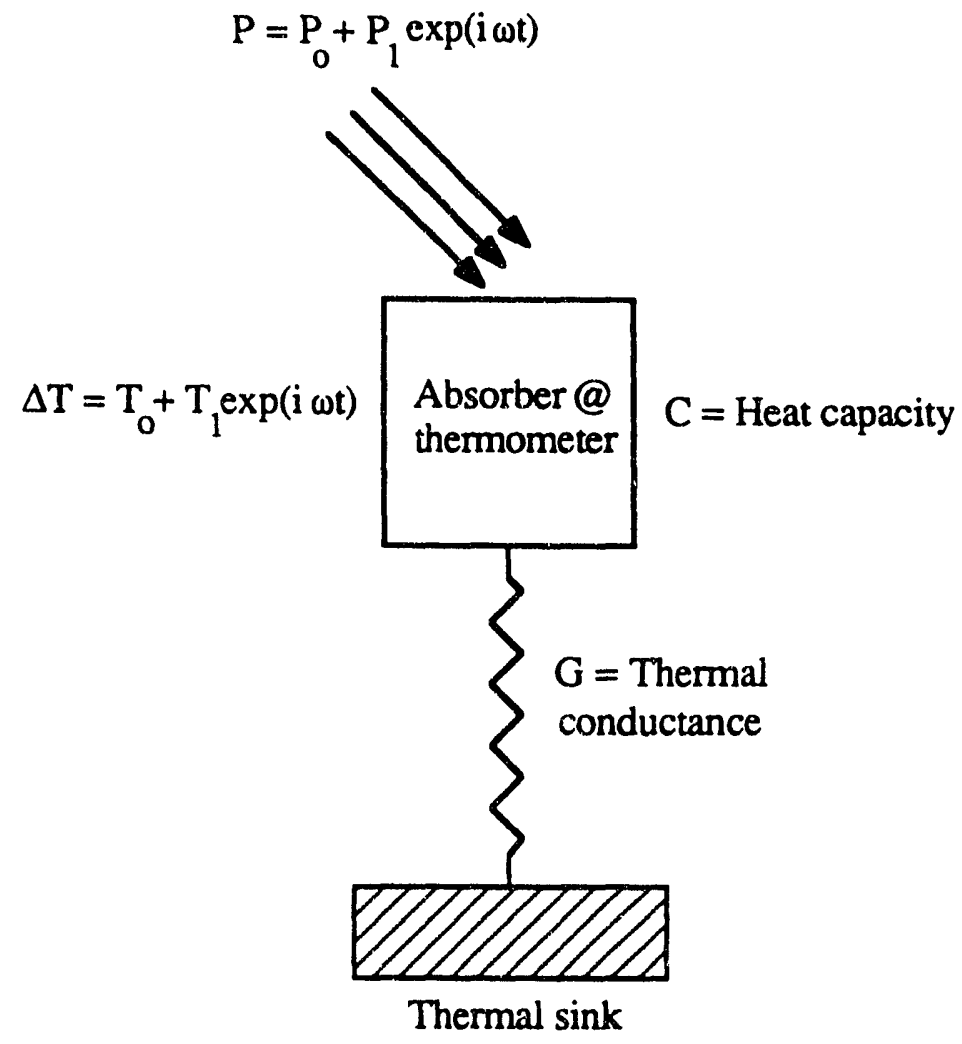

Fig. 2.1 Simplified thermal model for a bolometer. The thermally active region consists of an if absorber and a thermometer to measure the temperature rise $\Delta T$. The speed of response is determined by the ratio $C / G$, while the ultimate sensitivity is determined by the thermal conductance $G$. A sensitive bolometer should have a low heat capacity, low thermal conductance, and be operated at low temperatures. 


$$
C \partial(\Delta T) / \partial t+G \Delta T=P_{0}+P_{1} \exp (i \omega t)
$$

where $T_{s}$ is the sink temperature, and $\omega$ is the modulation frequency. The average operating temperature of the bolometer is found by equating the time independent terms and gives $T_{0}=T_{S}+P_{0} / G$. The temperature rise at the modulation frequency $T_{1}$ is found by equating the time varying terms to give

$$
T_{1}=P_{1} /(i \omega C+G)
$$

Thus far, the analysis has been indepencen: of the temperature readout scheme. The majority of bolometers use the temperature dependence of the resistance of semiconductors or of transition-edge superconductors as sensitive thermometers. The voltage across the thermometer can be written to first order as $V=I R(T) \approx I R_{0}+I(\Delta T) d R / d T$, where $I$ is the current bias and $\mathrm{dR} / \mathrm{dT}$ is the temperature coefficient of resistance. The time varying component of the measured voltage $\mathrm{V}_{1}$, can be measured by sensitive lock-in techniques at the modulation frequency $\omega$. The voltage responsivity of the bolometer is then defined as the change in the voltage across the thermometer per unit power that is absorbed $\mathrm{S}=\mathrm{dV}_{1} / \mathrm{dP}_{1}$, and is given by

$$
S=\frac{I d R / d T}{G(1+i(\omega \tau)}
$$

Here $\tau=C / G$ is the thermal response time of the bolometer.

Strictly speaking, this analysis is only valid when the bias heating and the effects of thermal feedback are both negligible. For the general case, it is easily shown that the average operating temperature is raised by an additional amount $\Delta T=I^{2} R_{0} / G$. Thermal feedback modifies the previous analysis in the following way: When the temperature of the bolometer changes as a result of the incident power, so does the resistance of the 
thermometer, which in turn changes the temperature of the bolometer through the change in bias heating. The net effect can be incorporated as a modification of the thermal conductance from $G$ to $G_{\text {eff. }}=G-I^{2} d R / d T$. When $G_{\text {eff }}=0$ (and for $d R / d T>0$ ), the resistance of the thermometer and the temperature of the bolometer: increase without bound (until a stable configuration is reached), a condition known as thermal runaway. Throughout the remainder of this dissertation, I will assume that the bias current is sufficiently low so that the effects of bias current heating and thermal feedback can be ignored.

\subsection{Measurements of Bolometer Responsivity}

It is often necessary to measure the responsivity of the bolometer. The simplest approach is to dissipate a known amount of power in the bolometer and to measure the resultant voltage rise. This information is contained in the dc I-V characteristic of the bolometer. From the previous discussion, the voltage across the bolometer is $V=I R_{0}+I(\Delta T) d R / d T$. The temperature rise due bias heating is just $\Delta T=I^{2} R_{o} / G$, so that the $\mathrm{I}-\mathrm{V}$ relation is

$$
V=\mathbb{R}_{0}+\frac{I d R / d T}{G} I^{2} R_{0}
$$

The slope of the voltage $V$ versus the bias power $I^{2} R_{0}$ is just the voltage responsivity $S$. The efficiency of the optical system can then be measured by using a calibrated source of radiation (such as a blackbody source) and comparing the measured signal with the expected magnitude.

One concern with this type of measurement is due to the presence of non-thermal nonlinearities in the thermometer. Thus, in addition to the temperature dependence of the thermometer resistance, it is necessary to account for the possibility that the resistance of the thermometer also depends on the bias current. This effect has been observed in doped

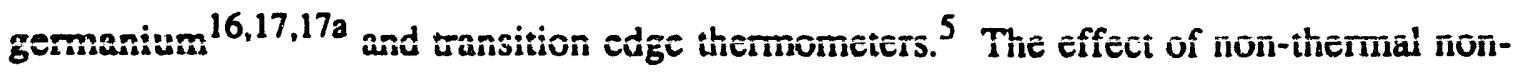


linearities can be incorporated in the previous analysis by expanding the thermometer resistance $R=R(T, I)$ in powers of temperature and bias current. As a consequence an additional term $(\partial \mathrm{R} / \partial \mathrm{I})_{\mathrm{T}} \mathrm{I}^{2}$ is added to the right hand side of Eq. 2.4, and complicates * measurement by introducing an additional non-thermal voltage offset. There does exist an elegant and simple method which to first order, can distinguish between these two effects. To the best of my knowledge, this method has not been discussed elsewhere. The idea stems from the fact that the voltage across the bolometer consists of a sum of three terms, each of which has a different functional dependence on the bias current $I$. The first term is just $R_{0} I$, the second term is $(\partial R / \partial I)_{T} I^{2}$, and the third is $R_{0}(\partial R / \partial T)_{I} G^{-1} I^{3}$. For a dc current bias it is difficult differentiate between the thermal and non-thermal contributions. If however, the bolometer is biased with an ac current $I=I_{0} \cos \omega_{0} t$, then to first order,

$$
\begin{aligned}
\mathrm{V} & =0.5(\partial \mathrm{R} / \partial \mathrm{I})_{\mathrm{T}} \mathrm{I}_{\mathrm{o}}{ }^{2} \\
& +\mathrm{R}_{\mathrm{o}} \mathrm{I}_{0} \cos \omega_{0} \mathrm{t}+0.75 \mathrm{R}_{\mathrm{o}}(\partial \mathrm{R} / \partial \mathrm{T})_{\mathrm{I}} \mathrm{G}^{-1} \mathrm{I}_{\mathrm{o}}{ }^{3} \cos \omega_{\mathrm{o}} \mathrm{t} \\
& +0.5(\partial \mathrm{R} / \partial \mathrm{I})_{\mathrm{T}} \mathrm{I}_{\mathrm{o}}{ }^{2} \cos 2 \omega_{0} \mathrm{t} \\
& +0.25 \mathrm{R}_{0}(\partial \mathrm{R} / \partial \mathrm{T})_{\mathrm{I}} \mathrm{G}^{-1} \mathrm{I}_{\mathrm{o}}{ }^{3} \cos 3 \omega_{0} \mathrm{t} .
\end{aligned}
$$

The thermal heating is manifested in the first and third harmonics, while non-thermal nonlinearities give rise to the signal at the second harmonic.

\subsection{Noise in Bolometers}

The various sources of noise in the bolometer will determine the minimum detectable power. The standard figure of merit that describes the sensitivity of bolometers is the NEP (noise equivalent power), which is the minimum detectable power in a one $\mathrm{Hz}$ noise bandwidth. For a bolometer the sources of noise are due to a) energy fluctuation noise in the active region, b) Johnson noise in the thermometer, c) amplifier noise, and d) all other sources of noise. An additional source of noise which is not inherent to the bolometer but 
which will, under certain circumstances, increase the output noise, is due to photon number fluctuarion in the incident radiation. ${ }^{18}$ In what follows, I will briefly review each of these sources of atise:

a) Energy fluctuation noise: Fluctuations in the energy of the active region give rise to fluctuations in the temperature, and hence in the output voltage of the bolometer. This noise is also referred to as thermal fluctuation noise, G-noise, or phonon noise. The source of these fluctuatiuns can be understood in terms of equilibrium thermodynamics. The thermal equilibrium mean square energy fluctuations in heat capacity $\mathbf{C}$ that is coupled to a heat sink at temperature $T_{S}$ through a thermal conductance $G$ is given by the following expression, 19

$$
<(\Delta E)^{2}>=\mathrm{kT}_{\mathrm{s}}^{2} \mathrm{C}
$$

The energy fluctuations give rise to temperature fluctuations $\left\langle(\Delta \mathrm{T})^{2}\right\rangle=k \mathrm{~T}_{\mathrm{s}}{ }^{2} / \mathrm{C}$, which appear as a voltage noise at the output of the bolometer. In practice, one uses sensitive lock-in techniques to measure the signal at the modulation frequency with a given frequency bandwidth (integration time). Thus, it is necessary to understand the spectral distribution of the temperature fluctuations. The standard analysis ${ }^{20}$ assumes a source of random power fluctuations $W(t)$, which is related to the temperature fluctuations via the heat equation

$$
\operatorname{Cd} \Delta T(t) / d T+G \Delta T(t)=W(t)
$$

The Fourier transform of Eq. (2.7) gives the spectral distribution of the temperature fluctuations, 


$$
\Delta T^{2}(\omega)=\frac{W^{2}(\omega)}{G^{2}\left(1+\omega^{2} \tau^{2}\right)},
$$

where $W(\omega)$ is the power spectral intensity of the fluctuations, and $\tau=C / G$. The spectral distribution of the temperature fluctuations is related to the mean square temperature fluctuations via the normalization

$$
\left.<(\Delta \mathrm{T})^{2}\right\rangle=\int_{0}^{\infty} \Delta \mathrm{T}^{2}(\omega) \mathrm{d} \omega
$$

We now assume that the frequency spectrum of $\mathrm{W}^{2}(\omega)$ is white and can be written as $\mathrm{W}^{2}(\omega)=\mathrm{B} \Delta \omega / 2 \pi$, where $\Delta \omega$ is the frequency bandwidth. Inserting this form into Eq. (2.9) gives $B=4 k T^{2} G$. In a one $\mathrm{Hz}$ bandwidth, $\left(4 k T^{2} G\right)^{1 / 2}$ then represents the rms power fluctuations in the bolometer, and thus represents the minimum power that can be detected by the bolometer in the absence of all other sources of noise.

b) Johnson noise in the thermometer: Johnson noise is caused by the random motion of thermally excited electrons in a resistor. For a resistance $\mathbf{R}$ in thermal equilibrium at a temperature $T$, the root mean square voltage fluctuations across the resistor are given by $\mathrm{V}_{\mathrm{n}}=(4 \mathrm{kTR})^{1 / 2}$. $^{21}$ The voltage fluctuations which appear at the output of the bolometer, can be related to a source of power fluctuations in the active region by the relation $\mathrm{NEP}=\mathrm{V}_{\mathrm{n}} / \mathrm{SI}$.

c) Amplifier noise: Excess noise in the first stage of amplification can often degrade the sensitivity of the bolometer and should thus be reduced to a level that is lower than the output noise of the bolometer. A common figure of merit for an amplifier is its noise temperature $T_{n}$. It is the temperature of the source resistor, whose Johnson voltage noise $\left(4 k T_{n} R\right)^{1 / 2}$, equals the amplifier noise. In general, the noise temperature of the amplifier depends on the source resistance $R$, and is considered to be unimportant when $T_{n}$ is smaller 
than the operating temperature. The fluctuation in power which corresponds to the noise in the amplifier is given by $\left(4 k T_{n} R\right)^{1 / 2} / S I$.

d) All other sources of noise: Excess noise in bolometers typically has a $1 / \mathrm{f}$ frequency spectrum and usually arises from a myriad of electronic processes in the thermometer. The sources of $1 / \mathrm{f}$ noise can arise from poor electrical contacts, material defects, or in the case of transition edge thermometers, from various flux flow mechanisms. Although much of the physics of $1 / \mathrm{f}$ noise is not well understood, it is generally believed that $1 / \mathrm{f}$ noise in bolometers can be controlled by improved fabrication techniques. It is customary to group theses various noise sources into an effective source of voltage noise $V_{1 / f}$. The corresponding fluctuation in power at the input of the bolometer is then given by $\mathrm{V}_{1 / \mathrm{f}} \mathrm{ASI}$.

The overall NEP for the power incident on the bolometer is given by the sum of the statistically independent contrih tions a) through d)

$$
N E P=\frac{1}{\eta}\left[4 k T^{2} G+\frac{4 k T R}{|S|^{2}}+\frac{4 k T T_{n} R}{|S|^{2}}+\frac{v_{1 / f}^{2}}{|S|^{2}}\right]^{1 / 2},
$$

where $\eta$ is the optical efficiency. For a sufficiently large responsivity, the contributions of the Johnson noise, amplifier noise, and $1 / \mathrm{f}$ noise to the NEP become negligible in comparison with the energy fluctuations in the active region. The phonon noise thus represents the ultimate limit to the sensitivity of the bolometer which is imposed by intrinsic thermodynamic fluctuations, and can be reduced only by lowering the operating temperature and/or by decreasing the thermal conductance.

The optimization of the bolometer for a given application depends on the operating temperature, background power loading, and modulation frequency. The trade-off between these requirements is not at all obvious. It is possible however, to make one general comment which is true for most applications. The heat capacity of the thermally active region and hence its volume, should be reduced as much as possible, thus enabling a 
simultaneous reduction of the thermal conductance without increasing the time constant of the bolometer. This simple concept stimulated much of the work described in this dissertation.

\subsection{Antenna-Coupled Bolometers}

The potential advantages of antenna coupled bolometers are best understood by considering the limitations of the competing technology. A conventional composite bolometer consists of a thin dielectric substrate and a thermometer which is usually glued onto the substrate. The electrical leads from the thermometer also provide the thermal conductance to the bath. A thin film absorber is usually deposited on the dielectric substrate. In order to couple radiation efficiently, the area of the absorber should approximately equal or be greater than the square of the wavelength of the incoming radiation. The only way to reduce the heat capacity of the bolometer then, is to thin the substrate. This can either be done by mechanical polishing techniques, ${ }^{22}$ or by fabricating thin membranes for the supporting substrate. ${ }^{23}$ In either case, the constraint on the area of the bolometer limits further reductions of the heat capacity.

A very different approach is to use thin film technology and lithographic patterning techniques to reduce the volume of the thermally active region to a fraction of a cubic micron. This should be compared to $\approx 10^{8} \mu \mathrm{m}^{3}$ for the volume of a conventional composite bolometer. The active region typically consists of an absorber to dissipate the rf currents in the antenna, and a thermometer to measure the resultant temperature rise. The thermometer can also act as the rf absorber and need not be a separate element. A comprehensive discussion of the sensing element will be postponed until later chapters. Millimeter wave radiation is coupled to the active region, which is much smaller than a wavelength, by means of an antenna, ${ }^{24}$ the properties of which will be discussed in the following section. 


\subsection{Antenna Theory}

An antenna is a mode converter for electromagnetic waves. For a typical measurement, the signal consists of electromagnetic waves that propagate in free space. The detecting element on the other hand, is usually mounted in an electromagnetic environment which is poorly matched to free space. The antenna can be regarded as a sort of 'transformer', taking one form of field pattern and converting it into another.

Antennas that are used for millimeter and submillimeter waves can be grouped into two broad categories. At low frequencies $(\mathrm{f}<300 \mathrm{GHz}$ ), feed horn antennas are often used to couple free space radiation to a waveguide which subsequently dissipates the if power in the detecting element (Fig. 2.2a). As the frequency is increased however, these structures become increasingly difficult to fabricate. Planar lithographed antennas (Fig. 2.2b) are now becoming the technology of choice for applications requiring operation at frequencies up to several THz, and array compatibility. All of the detector concepts described in this dissertation have incorporated the planar antenna concept.

The rommon figures of merit for antennas are a) Antenna pattern, b) Frequency response, c) Antenna impedance, and d) Antenna efficiency. ${ }^{25}$ In reality, these characteristics are mutually dependent, but for sake of clarity will be discussed separately. a) The variation in direction of the flux $P$ that is coupled to an antenna is called the antenna pattern, and is primarily influenced by the physical shape of the antenna. It is customary to use spherical coordinates to describe this pattern, thus $P=P(\Theta, \Phi)$. For many applications, it is desirable to radiate most of the power in a fairly narrow solid angle which is called the main lobe. The remaining power will radiate in other directions into what are called side lobes. Much effort in the development of antennas is devoted towards increasing the fractional power coupled to the main lobe.

b) An antenna usually responds to radiation within a frequency band which is determined by its physical geometry. For instance, the short wavelength limit of an otherwise lossless antenna is determined by the smallest features of the antenna structure. In addition, 


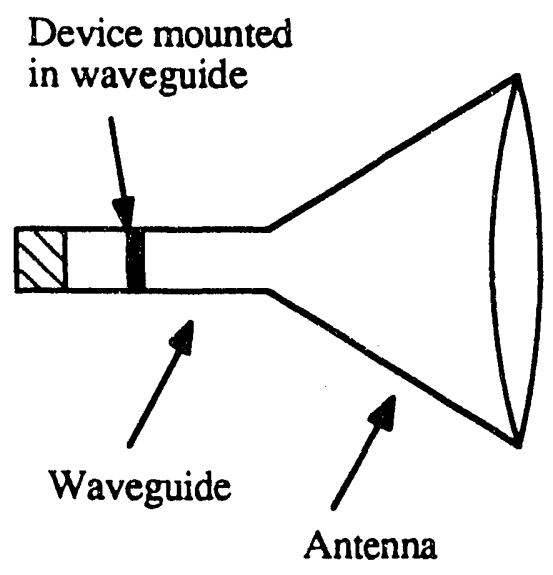

a)

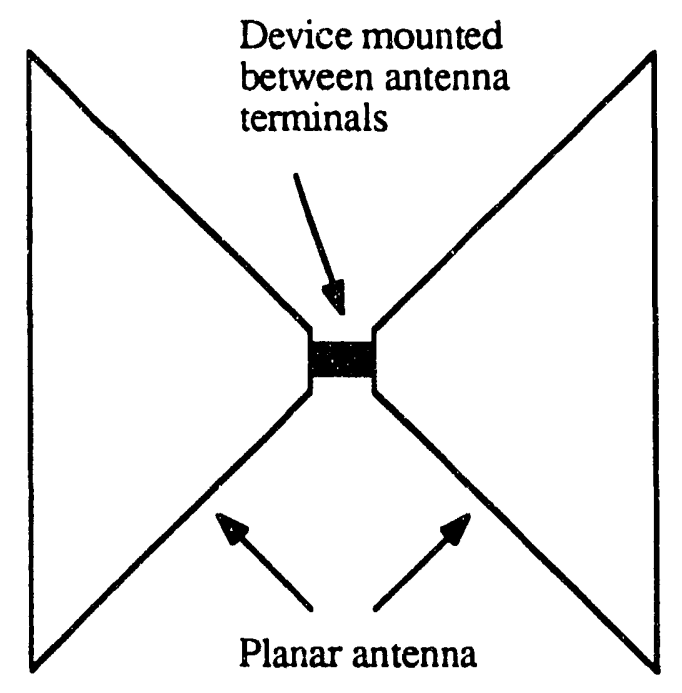

b)

Fig. 2.2 a) Combination of antenna, transmission line, and back-short arranged to couple a small device to an electromagnetic signal propagating in free space. b) Planar antenna used to couple radiation to a device that is located between the antenna terminals. All components are deposited directly on a substrate so that arrays can be conveniently produced by conventional lithographic techniques. The area of the active region is typically several square microns. 
resonant structures in the antenna can also influence the bandwidth.

c) In order that the active region dissipate all of the radiation that couples to the antenna, it is necessary that the if impedance of the absorber equal the complex conjugate of the if impedance of the antenna. This is an analogous to the case where a waveguide is terminated in a matched load. A useful case is when the antenna impedance is real and frequency independent.

d) The antenna efficiency is primarily influenced by rf losses. The issue is that an antenna which has a high surface resistance can dissipate if power which is subsequently made unavailable for detection. Thus, most antennas are fabricated from low loss metals such as copper or gold. It is also quite common to use superconducting antennas which have very low rf losses up to frequencies corresponding to the energy gap of the superconductor.

The ability to fabricate thin film structures with a sub-micron resolution, has enabled the development of a variety of novel planar-antenna structures which are deposited on a dielectric substrate. The main advantages of these antennas is ease of fabrication, array compatibility, and response at frequencies higher than those where feed horns and waveguide coupled structures cease to be useful. A very useful type of antenna is a selfcomplementary antenna, examples of which are shown in Fig. 2.3. A self-complementary structure is one in which the shape of the regions that are covered by metal is the same as the regions of the bare dielectric. The impedance of a planar antenna suspended in free space $Z_{\text {ant }}$, is related to the impedance of its complementary structure $Z_{\text {comp }}$ which is generated by replacing the metal regions with air and vice versa, via Booker's theorem ${ }^{26}$

$$
\mathrm{Z}_{\mathrm{ant}} \mathrm{Z}_{\text {comp }}=\mathrm{Z}_{\mathrm{o}}{ }^{2} / 4
$$

Here $Z_{0}=377 \Omega$ is the impedance of free space. A direct consequence of this theorem is that for a self complementary structure with $Z_{\text {ant }}=Z_{\text {comp, }} Z_{\text {ant }}=188.5 \Omega$ is real and 


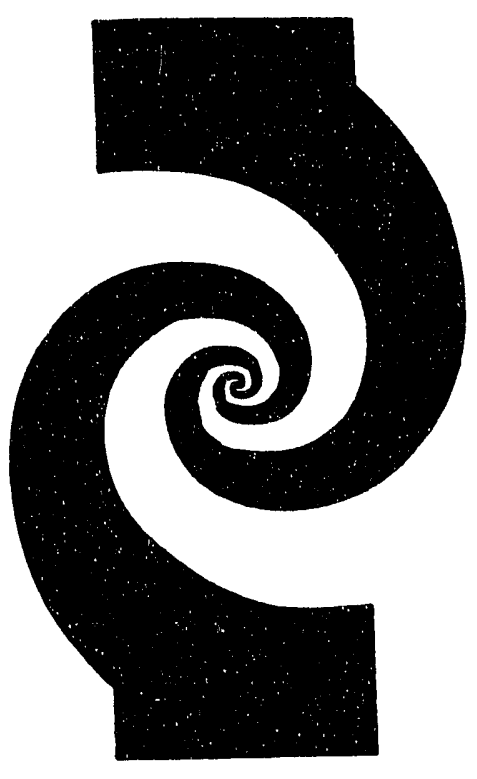

a)

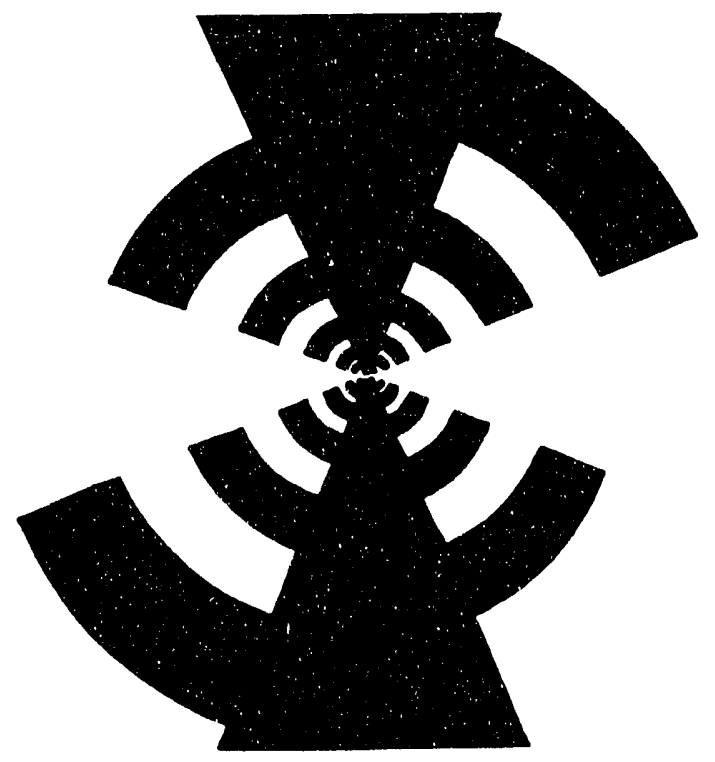

b)

Fig. 2.3 a) Sheet circular-toothed log-periodic antenna. b) Two-arm log-spiral antenna. 
independent of frequency. A self-complementary antenna thus has the potential of being very broadband. For a planar antenna located on a dielectric half space Booker's theorem is no longer valid because of the breaking of symmetry, but can be approximated by

$$
\mathrm{Z}_{\mathrm{ant}}=188.5 / 2^{1 / 2}(1+\varepsilon)^{1 / 2} \Omega
$$

where is $\varepsilon$ the dielectric constant of the substrate. ${ }^{24}$ For a quartz substrate $Z_{\text {ant }} \approx 120 \Omega$. Strictly speaking, $Z_{\text {ant }}$ will remain independent of frequency only for wavelengths which are of the same order as the smallest and largest features of the antenna that maintain the self-complementary structure.

An additional characteristic of planar antennas located on thick dielectric substrates is that they radiate preferentially through the substrate. ${ }^{24}$ This can be understood by considering a planar dipole antenna. The dipole measures the parallel component of the electric field. The local electric field at the antenna terminals is larger when the signal is incident from the dielectric side rather than from free space (due to the induced electric fields in the dielectric). The result is that the response of the dipole is larger when the wave is incident from the dielectric. A useful coupling scheme is to deposit the antenna on the back side of a dielectric lens, which can be used to further collimate the beam pattern. A variety of quasi-optical coupling techniques have been proposed and constitute an active field of research.

An important difference between antennas and conventional absorbers (such as an absorbing film) is in the manner in which they couple to radiation, and in particular to thermal radiation. An antenna couples to one spatial radiation mode and one polarization, whereas a conventional absorber can couple to many spatial radiation modes and two polarizations. This difference can be understood in the following manner: The rf currents in an antenna are constrained to flow only along one direction at the feed of the antenna, and thus have only one degree of freedom. A conventional absorber on the other hand has 
a very large number of degrees of freedom because it is comprised of a very large number of randomly oriented oscillators (atoms) that couple to the radiation field. Since bolometers are frequently used to detect thermal radiation, I will briefly summarize some standard results from thermal physics. The power per mode in a frequency bandwidth $d v$, from a blackbody maintained at temperature $\mathrm{T}$ is, ${ }^{19}$

$$
\mathrm{P}=\frac{\mathrm{hvdv}}{\exp (\mathrm{h} v / \mathrm{kT})-1}
$$

For a conventional absorber with an optical throuput $A \Omega$ equal to the product of the area $A$ at the beam focus times the angle $\Omega$ of divergence from the focus, the power radiated from the blackbody is

$$
P=\frac{2 A \Omega h v^{3} d v}{c^{2}[\exp (h v / k T)-1]}
$$

where $\mathrm{c}$ is the speed of light. ${ }^{19}$ The quantity $2 A \Omega \lambda^{2}$ (where $\lambda v=c$ ) can thus be interpreted as the number of spatial modes that couple to the absorber. In a frequency bandwidth $\mathrm{dv}$, and for a constant throughput, the ratio of the power that couples to a conventional absorber to the single mode antenna power is proportic.nal to $v^{2}$. The limitation to one spatial mode for the antenna coupled bolometer is an advantage for diffraction limited observations over a broad spectral range, where it is often necessary to efficiently reject short wavelength background radiation. Thus, antenna couplid bolometers can be more useful for diffraction limited broadband observations, whereas conventional composite bolometers would be more useful for broadband, constant throughput spectroscopic applications.

In chapter 5, I will present measurements of the frequency dependent optical efficiency of a self-complementary, log-periodic antenna and will discuss in further detail several of the issues which were mentioned here. 


\section{Chapter 3:_High- $T_{f}$ Microbolometer}

\subsection{Introduction}

Bolometers that use the resistive transition of high temperature superconductors as a sensitive thermometer are of much interest since there are many applications for infrared detectors, at wavelengths $>200 \mu \mathrm{m}$, where liquid nitrogen temperatures are acceptable and liquid helium temperatures are not. Applications include laboratory infrared spectrometers and long term observation of the earth or nearby planets from long lived space platforms. To date however, there has not existed a suitable technology for thermal detectors operating near $77 \mathrm{~K}$. Consequently, thermal detectors operated at room temperatures are often used in applications which would greatly benefit from the improved performance of a properly optimized detector operated near $77 \mathrm{~K}$. The performance of an ideal composite bolometer operated at $90 \mathrm{~K}$, with a heat sink temperature of $77 \mathrm{~K}$ was calculated by Richards et al. in $1989 .^{3}$ They estimated a noise equivalent power that is 100 times better than the $\approx 5 \times 10^{-10} \mathrm{WHz}^{-1 / 2}$ available from commercial pyroelectric detectors operated at room temperatures. The antenna-coupled high- $T_{c}$ bolometer (referred to as the high- $T_{c}$ microbolometer) was proposed by $\mathrm{Hu}$ et al. in $1989 .^{4}$ The potential advantages of this particular configuration is the low NEP, short time constant, and array compatibility. In their proposed design a planar lithograpted antenna is used to couple the radiation to a thin YBCO film with dimensions $\left(\approx 6 \times 13 \mu \mathrm{m}^{2}\right)$ which are much smaller than the wavelength to be measured. This film acts both as the resistor to thermalize the rf currents and as a transition edge thermometer to measure the resulting temperature rise. Because of its small size, both the thermal conductance from the film into the bulk of the substrate and the heat capacity of the thermally active region are small. Consequently, the microbolometer has low noise, fast response and a high voltage responsivity. This subject of this chapter is the fabrication and measurement of the high- $\mathrm{T}_{\mathrm{c}}$ microbolometer. I will review the basic operating principles of the microbolometer, describe the fabrication process, and present 
the experimental results. I will conclude with a discussion of the current limitations of the high- $\mathrm{T}_{\mathrm{c}}$ microbolometer, and possibilities for additional improvements.

\subsection{Energy Dissipation}

The significant reduction in the heat capacity of the thermally active region relative to that of conventional composite bolometers is one of the main advantages of this device. The thermal time constant of the microbolometer is $\approx 10^{-6} \mathrm{~s}$, which is much shorter than is required for most applications. Consequently, the performance of the microbolometer can be improved by reducing the thermal conductance from the film to the environment, without being restricted by speed of response considerations.

At temperatures above $\approx 30 \mathrm{~K}$, the thermal isolation is primarily controlled by the flow of heat from the small area YBCO film to the bulk of the substrate. Following the analysis of Ref. 4 , the thermal conductance $G$ from the superconducting film to the bulk of the substrate can be written as the following integral

$$
G(\omega)=\frac{\kappa}{\Delta T} \int_{\text {area }} \nabla T(r) d s,
$$

where $\kappa$ is the thermal conductivity of the substrate, $T(r)$ is the position dependent temperature in the substrate, $\Delta \mathrm{T}$ is the temperature difference between the film and the substrate far from the film, and the integral is over the contact area between the film and the substrate. If we model the contact between the film and the substrate as a hemisphere of radius a such that $A=2 \pi \mathrm{a}^{2}$ is the area of the film, then the radial diffusion equation gives

$$
T(r)=T_{s}+\Delta T(a / r) \exp [(a-r) / L] \exp [i(\omega t-r / L)]
$$

where $T_{S}$ is the sink temperature, $\left.L=(2 k i \omega)\right)^{1 / 2}$ is the thermal diffusion length, $c$ is the substrate heat capacity per unit volume, and $\omega=2 \pi f$ is the modulation frequency. The 
frequency dependent thermal conductance calculated from Eq. 3.1 is then given by

$$
G(\omega)=\kappa(2 \pi A)^{1 / 2}\left[\left(1+\tau^{1 / 2} \omega^{1 / 2}\right)^{2}+\tau \omega\right]^{1 / 2},
$$

where $\tau=c A / 4 \pi K$ is an effective time constant. At low frequencies, $G(0)$ is fr. quency independent while for fiequencies much higher than $1 / \tau, G \propto \omega^{1 / 2}$.

The contact area between the superconducting thermometer and the antenna edges provides an alternate path for heat dissipation. This additional contribution to the total thermal conductance is difficult to calculate precisely. However, previous estimates 27 and present measurements indicate that this is not the dominant thermal conduction path.

If we model the thermal circuit as a heat capacity $C$ coupled to a heat bath through a conductance $G(\omega)$, then the voltage responsivity is given by

$$
S=\frac{I(d R / d T)}{G(\omega)+i \omega C}
$$

where $I$ is the bias current, $d R / d T$ is the temperature coefficient of resistance, and $\omega$ is the modulation frequency. The second term in the denominator can be neglected because of the small thermal mass of the film. From the previous discussions it is seen that the best performance is obtained for a small film area deposited on a low thermal conductivity substrate.

\subsection{Radiation Coupling}

It is necessary to understand the if properties of the YBCO strip in order to design an efficient match to the antenna. Miller et al. ${ }^{28}$ have made direct measurements of the absortivity of YBCO films and concluded that the if impedance is adequately described by the two fluid model up to $\approx 15 \mathrm{THz}$. In this model, the surface impedance of a film of arbitrary thickness is given by the following expression, 29 


$$
\mathrm{Z}_{\mathrm{s}}=\frac{i 2 \pi v \mu_{0} \operatorname{coth}\left[\mathrm{t}\left(1 / \lambda^{2}+2 \mathrm{i} / \delta^{2}\right)^{1 / 2}\right]}{\left(1 / \lambda^{2}+2 \mathrm{i} / \delta^{2}\right)^{1 / 2}}
$$

Here $\lambda=\lambda_{\mathrm{o}}\left[1-\left(\mathrm{T} / \mathrm{T}_{\mathrm{c}}\right)^{4}\right]^{-1 / 2}$ is the superconducting penetration depth, $\delta=\left[1 / \mu_{0} \sigma_{n}\left(T / T_{c}\right)^{4} \pi v\right]^{1 / 2}$ is the classical skin depth calculated for the normal carriers below $T_{c}, \sigma_{n}$ is the conductivity in the normal state, $t$ is the film thickness, and $v$ is the $\mathrm{rf}$ frequency. This model is valid in the normal state if we set $T / T_{c}=1$ and $\lambda=\infty$. When the film thickness $t<<\delta$, the normal state surface impedance from Eq. (4) is just the dc resistance per square of the strip $Z_{s}=\left(t \sigma_{n}\right)^{-1}$. For a YBCO strip biased at the midpoint of the resistive transition, it is then reasonable to assume that dc resistance per square of the film is approximately equal to the $\mathrm{rf}$ surface impedance. A more detailed discussion of this - issue will be presented in chapter 5.

The most straightforward thermometer design is a simple strip of length $l$, width $w$. and thickness $t$, located directly between the antenna terminals. The impedance of such a strip is

$$
\mathrm{Z}_{\text {bolo }}=\mathrm{Z}_{\mathrm{S}} l / w+\mathrm{Z}_{\mathrm{L}}
$$

where $\mathrm{Z}_{\mathrm{L}}$ is the impedance due to the geometrical inductance of a rectangular ihin strip over a dielectric half plane and is approximately given by $\mathrm{Z}_{\mathrm{L}}=\mathrm{i} 1.5 \times 10^{-12} \mathrm{vl}$ where $\mathrm{l}$ is in $\mu \mathrm{m}$ and in $\mathrm{Z}_{\mathrm{L}}$ ohms. ${ }^{30}$ The ratio of the power coupled into the strip to the power available at the antenna terminals is given by the usual ratio of antenna and sensor impedances can be written as $4 Z_{\text {ant. }} R\left[\left(Z_{\text {ant. }}+R\right)^{2}+\left|Z_{L}\right|^{2}\right]^{-1} \cdot 31$ The coupling of $\mathrm{rf}$ currents into the strip will thus weakly depend on frequency for frequencies such that $\left|Z_{L}\right|<Z_{\text {ant. }}$. For tynical strip parameters, the frequency at which $\left|Z_{\mathrm{L}}\right|=\mathrm{Z}_{\mathrm{ant}}$ is $\approx 3 \mathrm{THz}$. The rf matching problem is thus reduced to matching the dc resistance of the strip to the real antenna impedance. 
The antenna used for this investigation was a self complementary equiangular spiral antenna which has an impedance of $75 \Omega$ for a $\mathrm{ZrO}_{2}$ substrate. ${ }^{32}$ Since planar antennas located on a dielectric surface radiate primarily into the dielectric, the signals are introduced through the back surface of the dielectric which is often placed on the back side of a dielectric lens.

\subsection{Device Fabrication}

In order to maximize the sensitivity of the microbolometer several conditions must be met. First, the resistance of the superconducting thermometer, when maintained at the center of its resistive transition, must match the antenna impedance of $\approx 77 \Omega$. Since high quality films of YBCO have typical resistivities of $\approx 100 \mu \Omega$-cm just above the resistive transition, film thicknesses of $\approx 1000 \AA$ are required. The second requirement is that of a low thermal conductivity substrate which is simultaneously compatible with YBCO and is not lossy at infrared frequencies. $\mathrm{ZrO}_{2}$ stabilized with $\mathrm{Y}_{2} \mathrm{O}_{3}(\mathrm{YSZ})$ has favorable thermal and film growth properties. It has a thermal conductivity $\mathrm{K}=0.015 \mathrm{Wcm}^{-1} \mathrm{~K}^{-1}, 33$ a specific heat $c=0.7 \mathrm{Jcm}^{-3} \mathrm{~K}^{-1}, 34$ and a dielectric constant $\varepsilon=12.5^{35}$

A collaborative effort was established with Conductus Inc. in which the films would be deposited at Conductus, whereas the devices would be patterned and tested at Berkeley. In situ, off axis sputtered YBCO films $(\approx 1000 \AA$ thick) were deposited on YSZ substrates. ${ }^{36}$ Subsequently, $\approx 2500 \AA$ of silver were deposited without breaking vacuum. The antenna pattern was defined using standard photolithographic techniques and the silver was etched in a $1 \mathrm{H}_{2} \mathrm{O}_{2}: 1 \mathrm{NH}_{4} \mathrm{OH}: 2 \mathrm{CH}_{3} \mathrm{OH}$ solution. The $\mathrm{YBCO}$ microbridge was then defined and etched in a $\approx 0.5 \%$ phosphoric acid etch. The resulting structure had an active area of $\approx 6 \times 13 \mu \mathrm{m}^{2}$ with a transition width of $\approx 2 \mathrm{~K}$ and a resistance of $\approx 40 \Omega$ at the center of the transition (Fig. 3.1). The substrate was mounted onto the back side of the hyperhemispherical sapphire lens with Apiezon $\mathrm{N}$-grease $\mathrm{s}^{37}$ and electrical contacts were made at the antenna terminals with silver paint. The temperature of the substrate-lens 


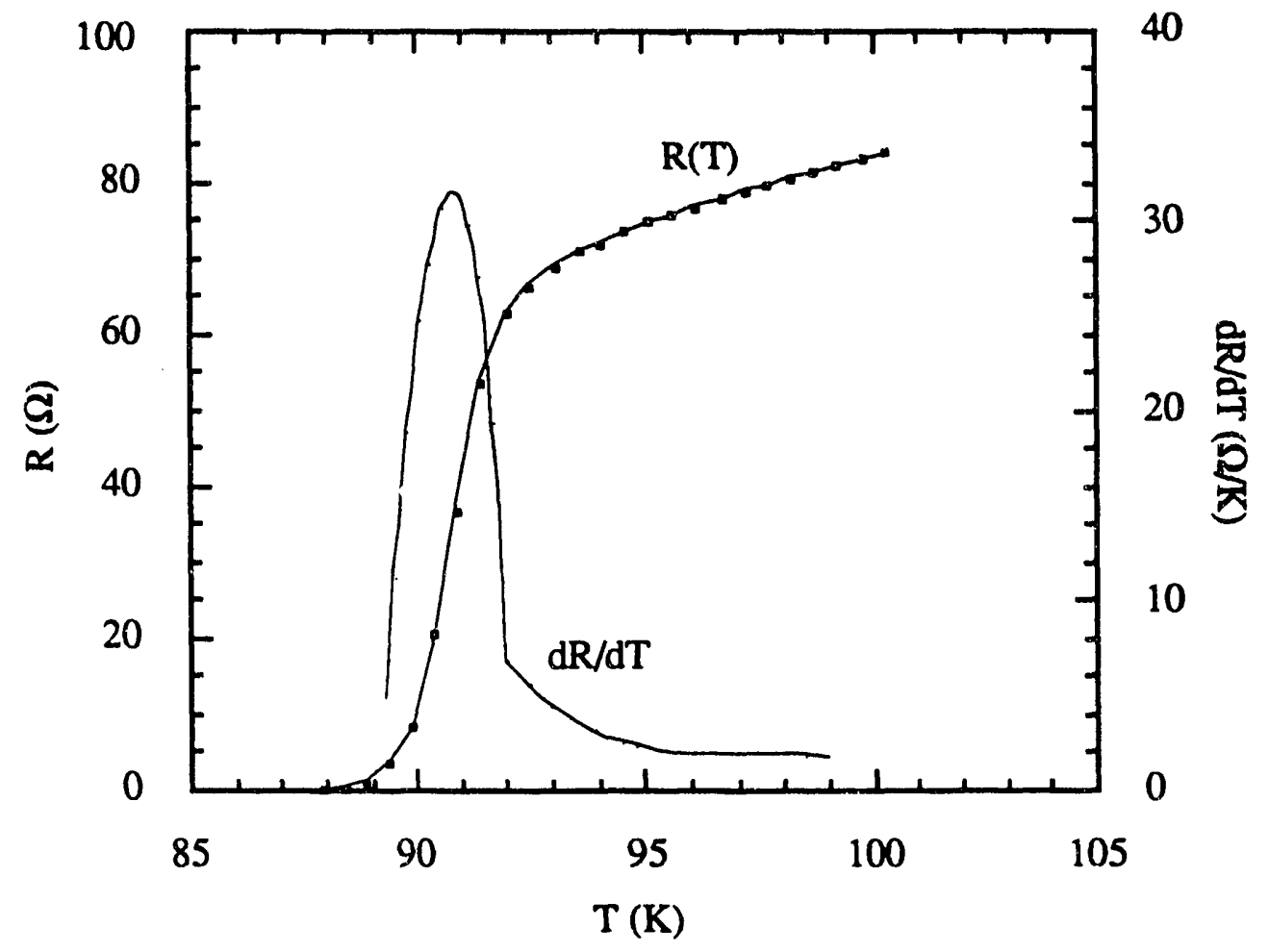

Fig. 3.1 Temperature dependence of the resistance of a typical microbolometer (measured at a bias of $100 \mu \mathrm{A}$ ), also plotted is the temperature coefficient of resistance whose maximum determines the optimum operating temperature. 
combination was regulated at the center of the resistive transition using a commercial diode thermometer and controller. ${ }^{38}$

\subsection{Electrical and Optical Characterization}

In order to calibrate the responsivity of the microbolometer it is necessary to measure the total thermal conductance from the thermally active region to the environment. As previously discussed, this information is contained in the dc I-V curve. The electrical power dissipated in the thermometer raises its temperature which results in a higher resistance. When the temperature was regulated at the center of the transition, a strong linear dependence of the resistance on the bias current was observed. This non-thermal behavior dominates the thermal effects. Thus, I-V curves were measured just above the resistive transition. Fig. 3.2 shows a typical resistance versus power load curve. The slight curvature is due to the change in $\mathrm{dR} / \mathrm{dT}$ as the thermometer changes temperature. From the slope we obtain $\mathrm{G}(0)=3.6 \times 10^{-5} \mathrm{~W} / \mathrm{K}$, while Eq. 3.3 predicts a value of $3.3 \times 10^{-5} \mathrm{~W} / \mathrm{K}$. The difference may be due to conduction of heat through the antenna. For a bias current of $600 \mu \mathrm{A}$, the voltage responsivity is $\approx 580 \mathrm{~V} / \mathrm{W}$.

The low impedance characteristic of antenna coupled devices necessitates the use of a transformer at the first stage of amplification. The electrical circuit that was used for the NEP measurements is shown in Fig. 3.3. A blocking capacitor $(C=100 \mu \mathrm{F})$ was used in conjunction with an Ithaco model 565 (1:10 turn ratio) low noise transformer and amplifier. ${ }^{39}$ With this configuration it was possible to bias the bolometer without passing a current through the transformer windings. A comprehensive discussion of the use of transformers as low noise amplifiers for source impedances $\leq 100 \Omega$ is given in App. A. The voltage noise, when referred to the input of the transformer was $0.1 \mathrm{nVHz}^{-1 / 2}$ between $0.01-25 \mathrm{kHz}$. The Johnson noise from the resistance of the microbolometer was a factor of $\approx 5$ higher.

In order to determine the nature of the noise, it is userud to plot the bias current 


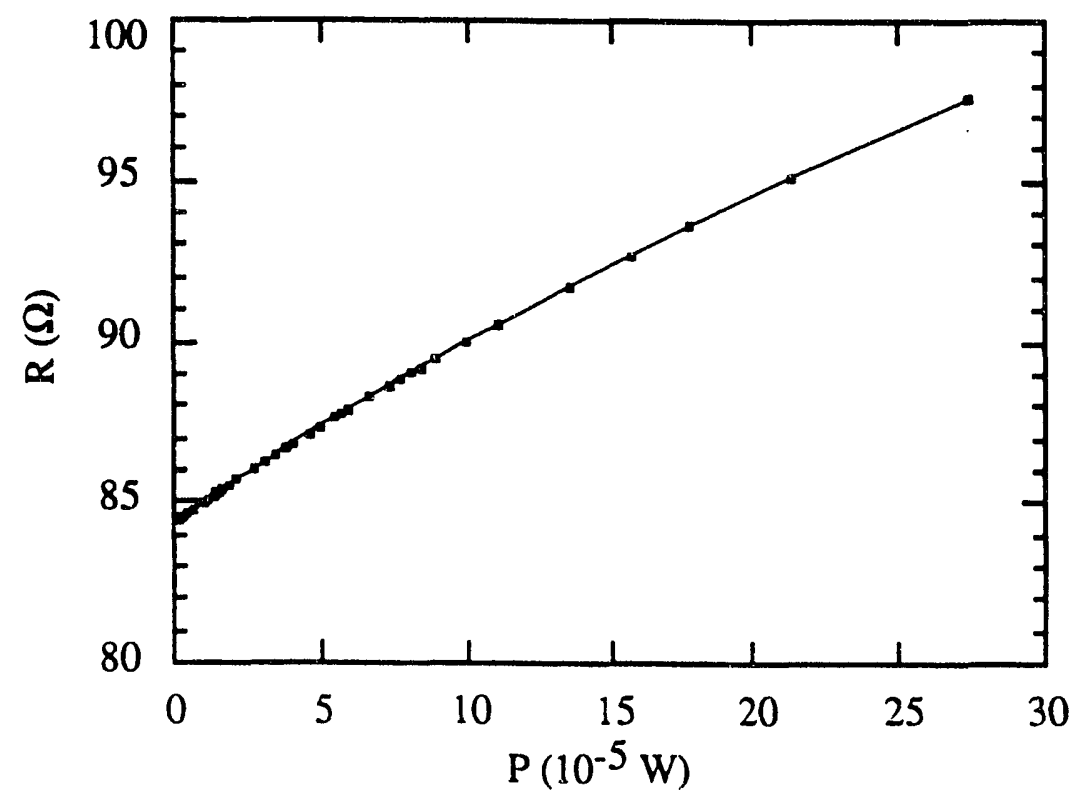

Fig. 3.2 Resistance versus power load curve measured just above the resistive transition. The slope of this curve equals $(\mathrm{dR} / \mathrm{dT}) \mathrm{G}^{-1}$. For a bias current of $600 \mu \mathrm{A}$, the voltage responsivity is $583 \mathrm{~V} / \mathrm{W}$. 


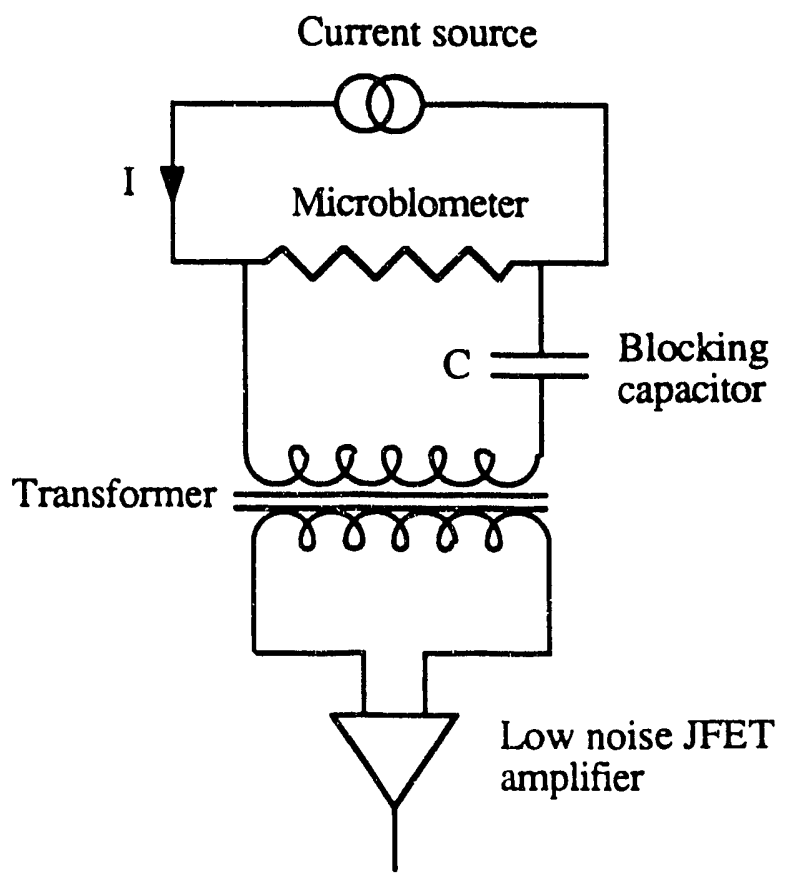

Fig. 3.3 Schematic of the microbolometer readout circuit For a transformer operating at $300 \mathrm{~K}$, the voltage noise is $\approx 0.1 \mathrm{nVHz}^{-1 / 2}$. A comprehensive discussion of transformers as low noise amplifiers for source resistances between $1-100 \Omega$ is given in appendix A. 
dependence of the NEP which is deduced from the ratio of the output voltage noise and the measured voltage responsivity. Fig. 3.4 is a plot of the electrical NEP as a function of bias current. Superposed is the theoretical curve which was deduced from the area of the microbolometer, the thermal conductivity of the substrate, and the measured temperature coefficient of resistance (and assuming negligible contributions from 1/f and amplifier noise). The solid line is the theoretical phonon noise limit $\left(4 \mathrm{kT}^{2} \mathrm{G}\right)^{1 / 2}$. At a bias current of $\approx 600 \mu \mathrm{A}$, the sensitivity of the microbolometer is seen to be limited by intrinsic thermal fluctuations in the active region, this is the optimum limit for any thermal detector because it indicates that the contribution to the NEP from all other noise sources has been made negligible. Additional improvements in the sensitivity of the microbolometer are possible only by reducing the thermal conductance to the environment. This can be achieved by depositing the YBCO film on a substrate with a lower thermal conductivity, by patterning a smaller active area, by creating artificial thermal barriers between the film and the substrate, or by depositing the film on a thin membrane (this latter possibility will be discussed in section 3.7). It should be noted that the $\approx 2.6 \mathrm{nVHz}^{-1 / 2}$ voltage noise at the output of the microblmeter can be read out with conventional FET amplifiers which have a typical input voltage noise of $\approx 1.5 \mathrm{nVHz}^{-1 / 2}$. The current noise in the amplifier makes a small contribution to the total noise of the amplifier due to the small bolometer resistances.

The frequency dependence of the NEP is shown in Fig. 3.5 for a bias current of $500 \mu \mathrm{A}$. The solid line is the NEP expected in the absence of $1 / \mathrm{f}$ and amplifier noise. The exact origin of the excess noise at frequencies below $\approx 1 \mathrm{kHz}$ is not fully understood, but is believed to be influenced by the deposition and patterning process.

Measurements of the optical response of the microbolometer were made at $90 \mathrm{GHz}$ with a Gunn oscillator whose bias was electrically switched. The oscillator was coupled to a conventional feed horn via a waveguide. Radiation from the oscillator was directed towards the dewar window. We did not attempt to optimize the coupling efficiency since the signal from the oscillator was sufficiently high. In Fig. 3.6 we plot the optical response 


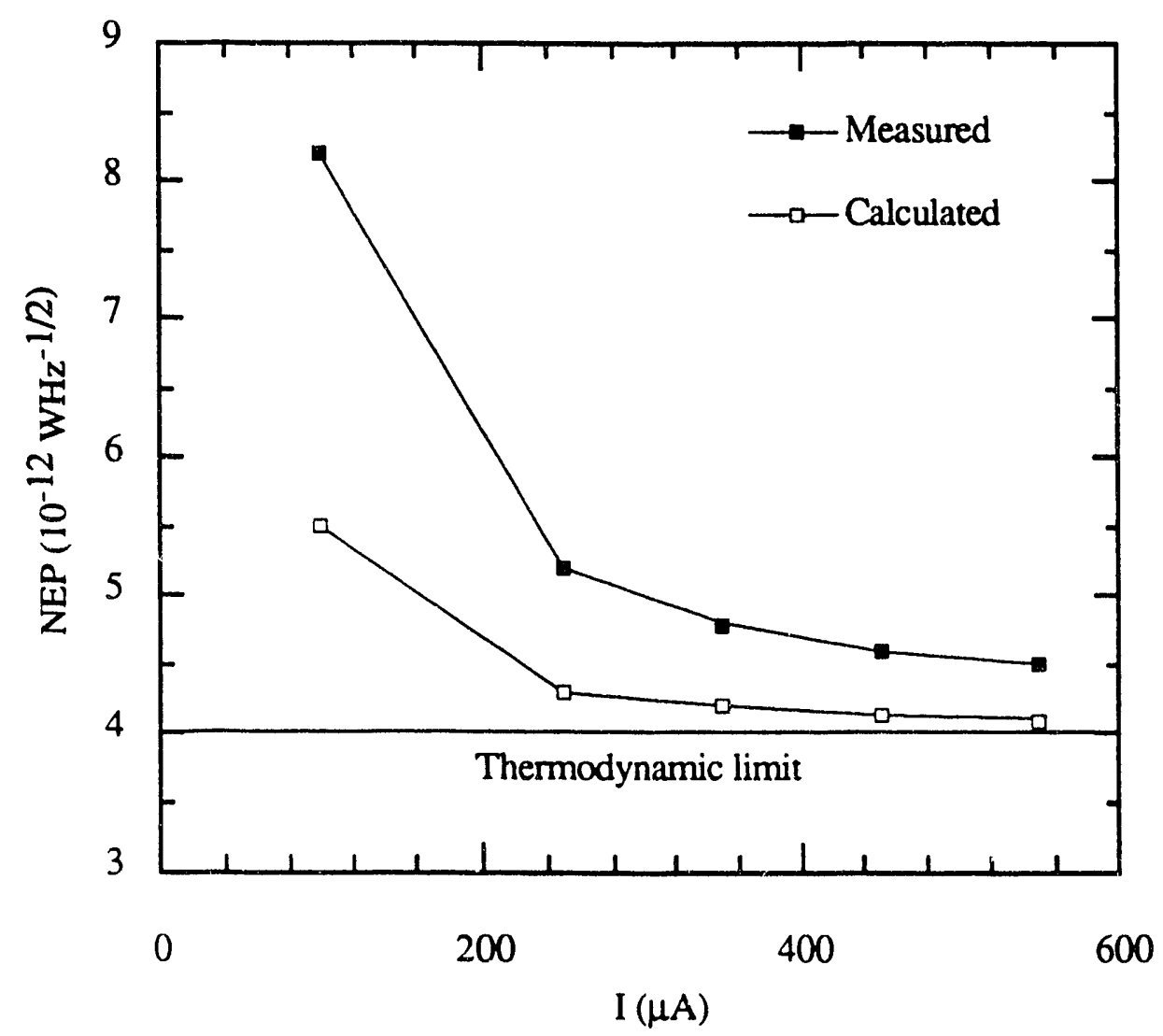

Fig. 3.4 The measured and calculated NEP (at $10 \mathrm{kHz}$ ). For a bias current larger than about $500 \mu \mathrm{A}$, the NEP is limited by intrinsic energy fluctuations in the YBCO strip, this is the ideal limit for a thermal detector. 


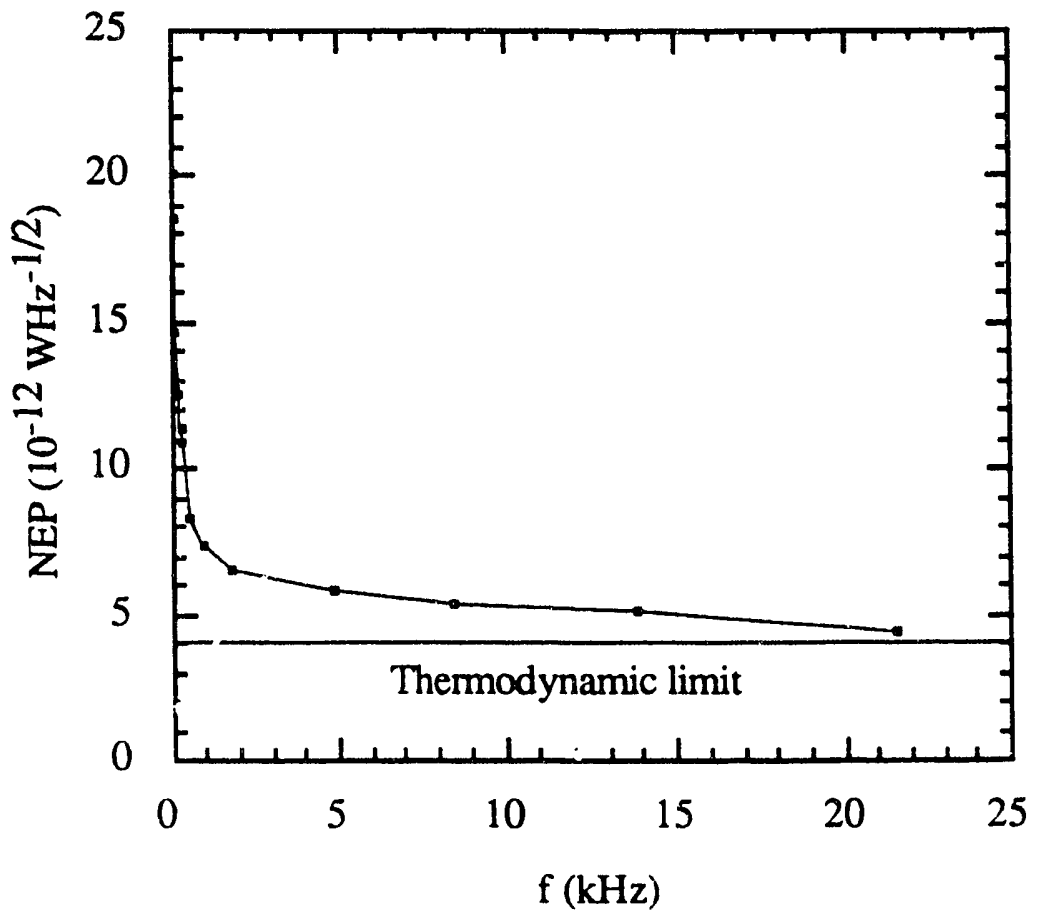

Fig. 3.5 Frequency dependence of the electrical NEP measured at the optimum operating temperature, and a bias of $100 \mu \mathrm{A}$. The solid line is the calculated NEP in the absence of $1 / \mathrm{f}$ noise. It is generally believed that improved fabrication and processing techniques will tend to move the $1 / \mathrm{f} \mathrm{knee} \mathrm{to}$ lower frequencies. 


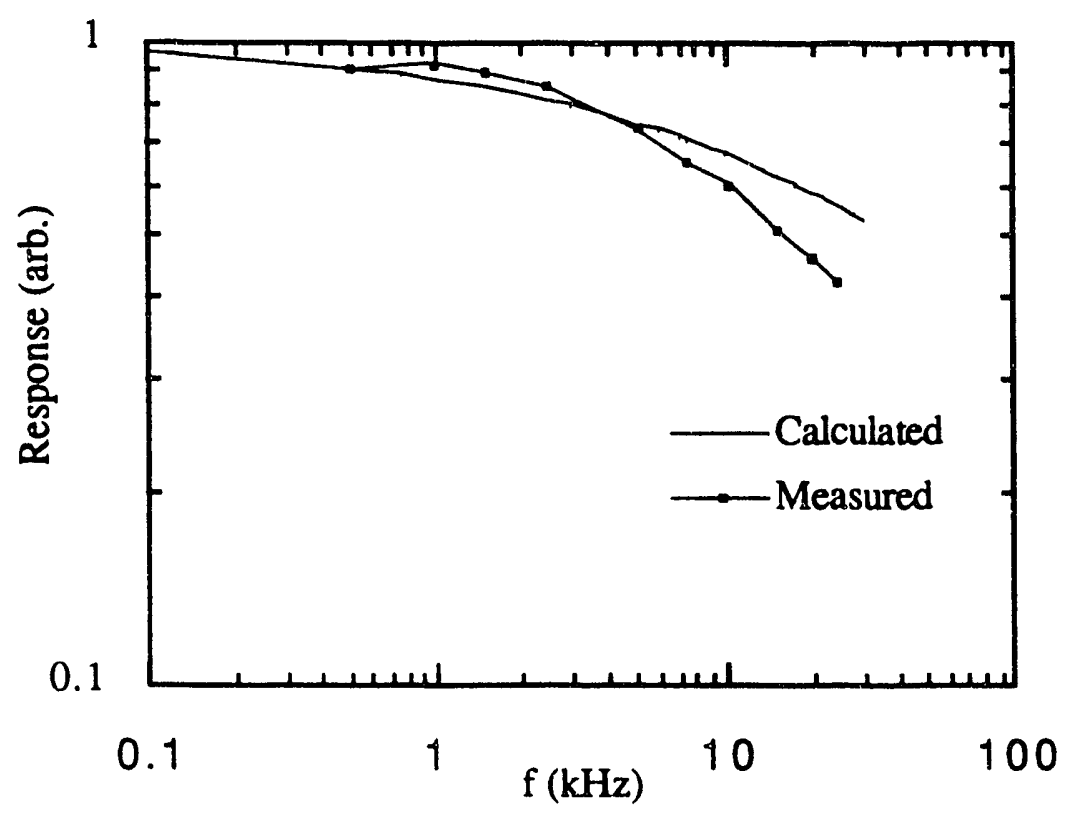

Fig. 3.6 Frequency response of the microbolometer at $90 \mathrm{GHz}$. The response calculated from the simplified thermal model agrees well with the measured response. 
of the microbolometer as well as the frequency dependence calculated from the thermal model discussed in section 3.2. The agreement is quite satisfactory if one takes into account the simplified nature of the model.

\subsection{Measurements of the Optical efficiency}

In order to accurately measure the optical efficiency, it is necessary to have a well calibrated source. In addition, the beam patterns of both the source and the detector must be known in order to estimate the coupling losses. A blackbody source appears to be a convenient choice. The power from the blackbody is well known and given by $P=k_{B} T B$ in the Rayleigh-Jeans limit, which is appropriate for this experiment. Here $\mathrm{T}$ is the temperature of the blackbody, and B is its optical bandwidth. ${ }^{19}$ Furthermore, it is possible to minimize the coupling losses by overfilling the beam pattern of the detector with the blackbody radiation.

In our configuration shown in Fig. 3.7, a sheet of Ecosorb AN72, 40 immersed in liquid nitrogen, serves as a $77 \mathrm{~K}$ blackbody source. The optical bandwidth $\mathrm{B}$ is determined by cooled fused quartz and black polyethylene filters, at the cryostat entrance, which had a measured effective bandwidth of $1.5 \mathrm{THz}\left(50 \mathrm{~cm}^{-1}\right)$. Thus, when chopped between $77 \mathrm{~K}$ and room temperature, the incident power is $\mathrm{P}=4.5 \times 10^{-9} \mathrm{~W}$. The integrated optical efficiency, defined as the ratio of the detected power to the incident power at the TPX lens, was measured to be $5 \%$. The coupling efficiency for a planar antenna suspended in free space is $50 \%$, and $\approx 75 \%$ when deposited on a dielectric. ${ }^{24}$ This indicates that the losses in the lens and antenna system were $\approx 15 \mathrm{~dB}$ over the frequency range $\left(0-50 \mathrm{~cm}^{-1}\right)$ of the observation. The observed losses can be accounted for as follows. The TPX lens had a measured frequency independent loss of $1 \mathrm{~dB}$. The losses of the YSZ/Apiezon Ngrease/Sapphire lens combination were measured to be $9 \mathrm{~dB}$ when normalized to the optical bandwidth. The main source of loss is in the $0.5 \mathrm{~mm}$ thick YSZ substrate, whose measured transmittance becomes negligible above $\approx 30 \mathrm{~cm}^{-1} .41$ 


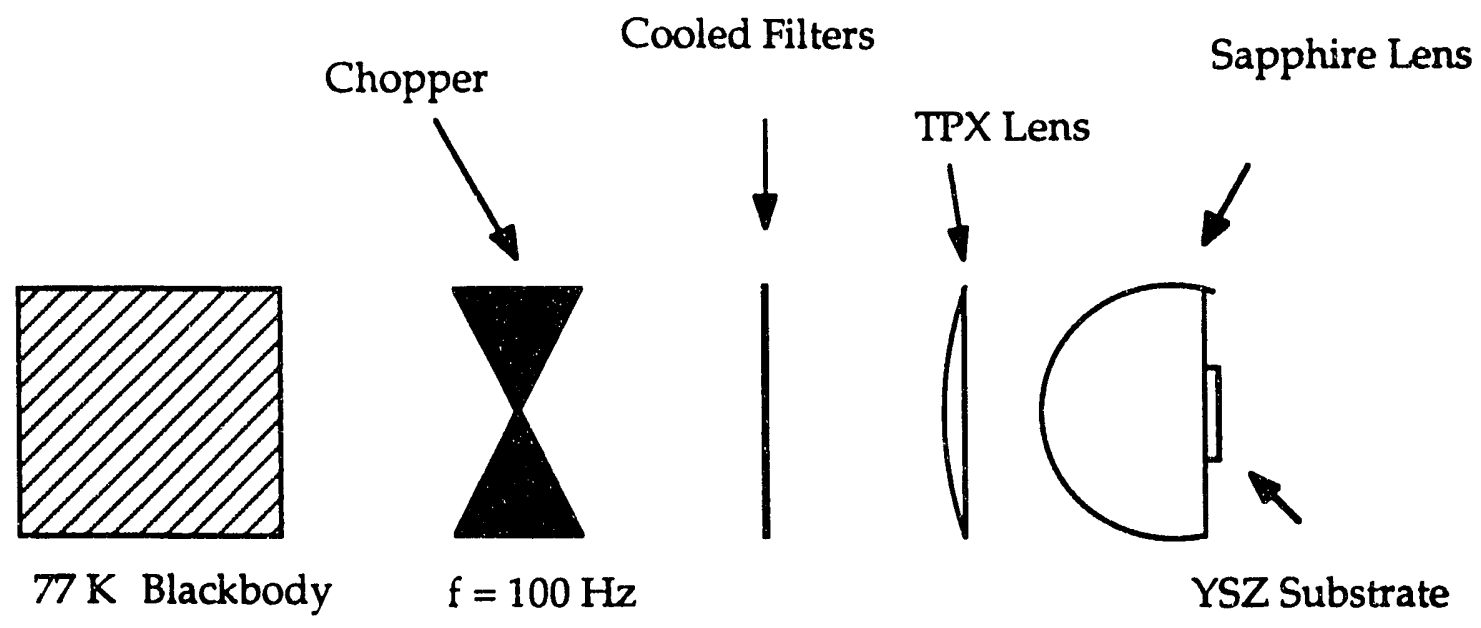

Fig. 3.7 Schematic of the experimental setup used to measure the optical efficiency of the quasi-optical systern. The substrate is mounted on the flat side of a hyperhemispherical sapphire lens $(D=0.5 ")$. A TPX lens $(f / 1.16)$ is used to further narrow the beam. 


\subsection{Conclusions}

To date, the electrical NEP of the microbolometer is the lowest of any other high- $\mathrm{T}_{\mathrm{c}}$ detector. Its sensitivity can be improved by further reducing the thermal conductance $G$ from the YBCO film to the substrate. This can be achieved by reducing the contact area of the YBCO film with the underlying substrate. Alternatively, it may be possible to deposit buffer layers between the film and the substrate, and subsequently increase the thermal isolation. This latter possibility will be investigated in the following chapter. In either case, the minimum permissible value of $\mathrm{G}$ is ultimately determined by the background power loading $P$ through the relation $P=G \Delta T$, where $\Delta T$ is the difference between the operating temperature and the heat sink temperature.

Significant improvements in the optical efficiency appear possible. Since the majority of the losses are due to absorption in the substrate, the use of a thinned YSZ substrate should reduce these losses. In addition, the use of reflecting optics, and perhaps a quartz lens should reduce the losses in the TPX lens and broaden the optical bandwidth (quartz is less lossy than sapphire at $\approx 100 \mathrm{~cm}^{-1}$ ). A better overall approach would be to deposit the antenna and the sensing element on a thin membrane. Because the membrane can be made much thinner than the detected wavelength, the antenna effectively radiates in free space, thus eliminating the substrate loss as well as the need for a dielectric lens. Measurements on log-periodic bismuth microbolometers deposited on a $1 \mu \mathrm{m}$ silicon-oxynitride membrane show no sidelobes and a $3 \mathrm{~dB}$ beamwidth of $40^{\circ} .42$ Because the thermometer is deposited on a thin membrane, the thermal conductance $\mathrm{G}$ is calculated in the two dimensional limit and, in general, will be lower than the three dimensional case, yielding a slower bolometer with a smaller NEP. 


\section{Chapter 4: Thermal Boundary Resistance for YBCO Films}

\subsection{Introduction}

Since the discovery of high- $T_{c}$ superconductivity, much attention has been focused on the response of thin superconducting films to pulsed radiation from visible to far infrared wavelengths. The motivations for these s:.periments were to develop sensitive radiation detectors, fast optical switches, and to gain a broader understanding of the physics of high$T_{c}$ superconductivity. A typical experimental configuration is shown in Fig. 4.1a. A thin film of YBCO is patterned into a narrow strip, typic ally $2.100 \mu \mathrm{m}$ wide. The strip is biased at a constant current $I$, and the vol'age $V$ across the strip is monitored. The time dependence of the response, after a short (ns) laser pulse incident on the strip, is shown in Fig. 4.1b. Observations of a variety of response times fror femtoseccnds to milliseconds with different temperature dependencies have caused some confusion about the response mechanisms. Several studies identify a bolometric response, due to heating of the film relative to distant parts of the substrate. ${ }^{43-45}$ Others have proposed mechanisms relating to vortex motion, ${ }^{6,7}$ phase slips, ${ }^{8}$ and non-equilibrium superconductivity 6,9 to account for what is claimed to be a nonbolometric response. Any attempt to model the thermal response, and hence to test the hypothesis of a nonbolometric mechanism, requires a quantitative knowledge of the thermal contact between the film and the substrate. ${ }^{46}$ This information has not generally been available to the community.

In the previous chapter, the properties of the high- $T_{c}$ microbolometer were analyzed in terms of the heat flow through the substrate. This analysis is quite valid for low modulation frequencies and for substrates with a low thermal conductivity. However, if one is trying to understand the transient response of YBCO films to very short laser pulses, it is necessary to understand and incorporate the effects of the thermal boundary resistance between the film and the substrate. It is unfortunate that much of the community was completely unaware of the importance of this effect. 


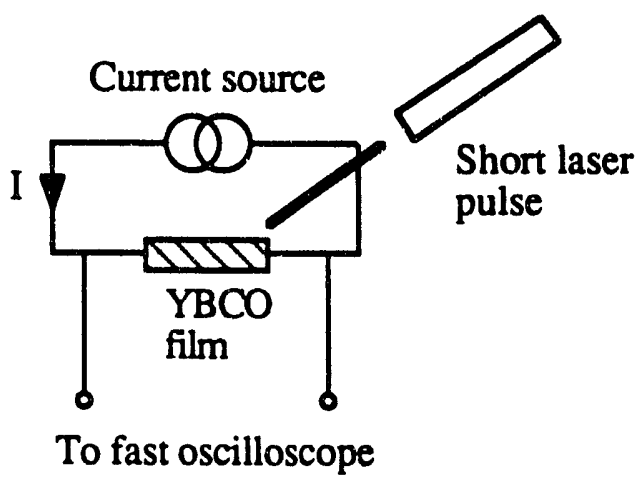

a)

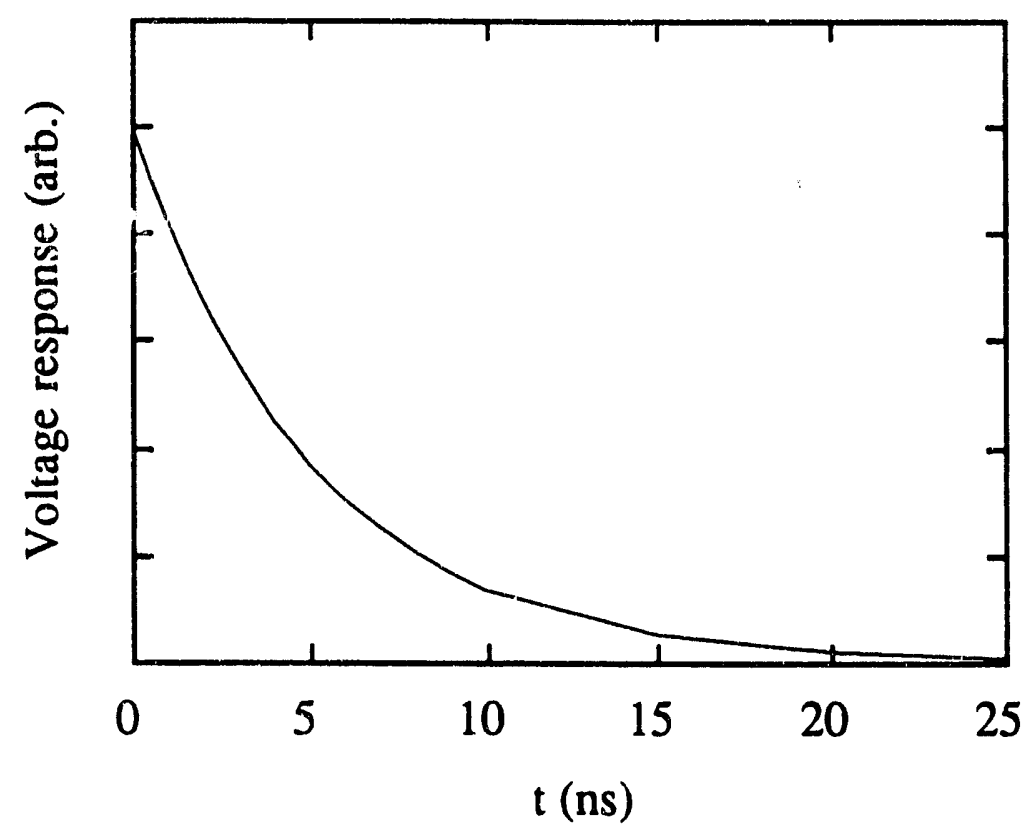

b)

Fig. $4.1 \quad$ a) Typical experimental setup for measurement of the response of thin YBCO films to pulsed laser radiation. b) Typical observed voltage response of a YBCO film to a laser pulse (ns duration). 
I will first give an overview of the thermal boundary resistance at low and high temperatures. I will then discuss the difficulties associated with the measurement of $R_{b d}$ at high temperatures, and present the experimental configuration which resolves these difficulties. Finally, I will conclude by arguing that the nanosecond response time of YBCO films can be entirely explained by the thermal boundary resistance, and will speculate on the origin of the shorter response times that are observed.

\subsection{Theoretical Background}

The thermal boundary resistance $R_{b d}$ is defined as the ratio of the temperature difference $\Delta T$ across an interface to the power per unit area P/A flowing across it,

$$
R_{\mathrm{bd}}=\Delta \mathrm{TA} / \mathrm{P}
$$

This definition is general and applies to both phonon and electron trarsport. We are interested in the case where at least one of the interfaces is a dielectric, so that only phonons can transport energy across the interface. The characteristics of the thermal boundary resistance are strongly dependent on the temperature and can not be described by one specific model. I will thus give a brief overview of the theory which is used to explain the boundary resistance at low temperatures, and also discuss our current understanding of the behavior of $R_{b d}$ at high temperatures .

At temperatures below $\approx 30 \mathrm{~K}$, the Acoustic Mismatch Model (AMM) developed by W. A. Little has been very successful in explaining thermal boundary resistances. ${ }^{47}$ The analysis is simple enough that it is worthwhile to present it here. The power flow across an interface (from side 1 to side 2) is the sum over all frequencies and incident angles of the number of phonons that are incident on a unit area $A$ per unit time, times the phonon energy $\hbar \omega$, times the transmission probability $\alpha_{1 \rightarrow 22}$. This can be written as 


$$
P_{1 \rightarrow 2}(T)=\frac{A}{4 \pi} \sum_{j} \iiint_{\Phi \Theta} \int_{\omega} N_{1, j}\left(\omega, T \hbar \omega \alpha_{1 \rightarrow 2}(\Theta, j) c_{1, j} \cos \Theta \sin \Theta d \Phi d \Theta d \omega .\right.
$$

Here $N_{1 j}(\omega, T)$ is the product of the phonon density of states (per unit volume) times the Bose-Einstein occupation factor in side 1 , the subscript $j$ is the phonon mode, $c_{1, j}$ is the phonon propagation velocity of mode $j$ in side 1 , and $\alpha_{1->2}(\theta, j)$ is the transmission probability for a phonon of mode $\mathrm{j}$ to be transmitted to side 2 . Here we have assumed that the transmission probability is independent of the phonon energy and bath temperature. The justification of Eq. (4.2) is simple, $N_{1, j}(\omega, T) c_{1, j} \cos \Theta d \Omega / 4 \pi$ is just the number of phonons with a given frequency and element of solid angle $d \Omega=\sin \Theta d \Phi d \Theta$ that are incident on the surface per unit time. Here $\Theta$ is the angle between the phonon propagation direction and the normal to the interface, and $\Phi$ is the azimuthal angle. The thermal boundary resistance is then given by

$$
R_{b d}=\frac{P_{2 \rightarrow 1}\left(T_{2}\right)-P_{1 \rightarrow 2}\left(T_{1}\right)}{A\left(T_{2}-T_{1}\right)}
$$

Thus far, the analysis has been independent of the operating temperature and the exact nature of the scattering mechanism at the interface, and thus applies equally well to all temperatures. Little was able to solve this model by making the assumptions that the propagation of the phonons is governed by continuum acoustics, and that the interface can be treated as a plane. These assumptions are expected to hold at low temperatures where the dominant phonon wavelengths of $\approx 400 \AA$ at $1 \mathrm{~K},{ }^{48}$ are much larger than a typical lattice spacing. The heart of the problem lies in the calculation of the transmission probability for the various phonon modes. In the continuum acoustic limit the transmission probability can be derived by imposing the appropriate boundary conditions on the values of the displacement and stress at the interface. These calculations are difficult since it is necessary 
to keep track of one longitudinal and two transverse phonon modes (for a one atom basis), and the possibility of mode conversion processes at the interface. I will not attempt to derive these probabilities but remark that they depend on the angle of incidence $\Theta$, and on ratios of the acoustic impedances $Z=\rho c$ of the two materials, where $\rho$ is the mass density. We can now derive the temperature dependence of the thermal boundary resistance at low temperatures. It is customary to assume that the solid is isotropic and to use the Debye approximation for the density of states. Thus, the number of phonons per unit volume, at temperature $\mathrm{T}$ and with an energy between $\hbar \omega$ and $\hbar \omega+\mathrm{d}(\hbar \omega)$ is given by 49

$$
N_{1, j}(\omega, T) d \omega=\frac{\omega^{2} d \omega}{\left.2 \pi^{2} c_{1, j}^{3}[\exp (\hbar \omega / k T)-1)\right]}
$$

One then proceeds by making the usual assumption that the Debye temperature is much higher than the temperature of interest, so that the integration over $\omega$ in Eq. 4.2 can be extended to infinity. The standard result for the boundary resistance then follows,

$$
\mathrm{R}_{\mathrm{bd}}=\left[\frac{\pi^{2} \mathrm{k}^{4}}{15 \hbar^{3}} \sum_{\mathrm{j}} \mathrm{c}_{1, \mathrm{j}}^{-2} \Gamma_{1, \mathrm{j}}\right]^{-1} \mathrm{~T}^{-3},
$$

where

$$
\Gamma_{1, j}=\int_{0}^{\pi / 2} \alpha_{1 \rightarrow 2}(\Theta, j) \cos \Theta \sin \Theta d \Theta .
$$

As previously discussed, the calculation of the transmission probability is difficult. The values of $\Gamma_{1, j}$ are usually tabulated as a function of the ratio of the two material densities, the longitudinal velocities, Poisson's ratio for each side, and the ratio of the longitudinal to transverse velocities on each side. One then calculates the coefficient of $\mathrm{T}^{-3}$ 
in Eq. (4.5). It is customary to group the various terms into one coefficient so that $\mathbf{R}_{\mathrm{bd}}=\mathrm{BT}^{-3}$. A general rule of thumb is that for most materials, $\mathrm{B}$ varies from about $6 \mathrm{~K}^{4} \mathrm{~cm}^{2} / \mathrm{W}$ (aluminum on quartz) to $90 \mathrm{~K}^{4} \mathrm{~cm}^{2} / \mathrm{W}$ (indium on diamond). For $\mathrm{YBCO}$ on sapphire I calculate $B=17 \mathrm{~K}^{4} \mathrm{~cm}^{2} / \mathrm{W}$.

At temperatures above $\approx 30 \mathrm{~K}$, the shorter phonon wavelengths $(\approx 4 \AA \text { at } 100 \mathrm{~K})^{48}$ are very sensitive to interface imperfections and irregularities. As a result the measur $\mathbf{R}_{\mathbf{b d}}$ does not continue to decrease as $T^{3}$, but approaches a constant value at high temperatures. At $100 \mathrm{~K} \mathrm{R}$ bd can exceed the ideal model predictions by several orders of magnitude. Although the exact details of this discrepancy are not well understood, it is generally argued that phonon scattering caused by disorder in the subsurface region is a strong candidate. ${ }^{50}$ Fig. 4.2 shows the typical temperature dependence of $R_{b d}$ at low and high temperatures.

Low temperature physicists are generally familiar with thermal boundary resistances. They play an important role in steady state experiments because they become important compared with bulk thermal conductances in many experimental situations. At higher temperatures, boundary resistances can often be neglected, but not when fast phenomena in thin films are involved.

\subsection{Measurement Scheme}

The goal of our experiments was to measure the magnitude of the thermal boundary resistance at $\approx 90 \mathrm{~K}$. We wanted to know whether $R_{b d}$ was significantly higher than the predictions of the AMM. The experiment would thus have to resolve at least $10 \%$ of the predictions of the AMM at and above $90 \mathrm{~K}$. This is not such an easy task at these temperatures because the thermal conductance across the interface can be several orders of magnitude higher than the thermal conductance from a typical distance through the substrate between adjacent thermometers.

Our measurements of the thermal boundary resistance use the technique reported by Swartz and Pohl. ${ }^{51}$ The experimental geometry, shown in Fig. 4.3, consisted of three 


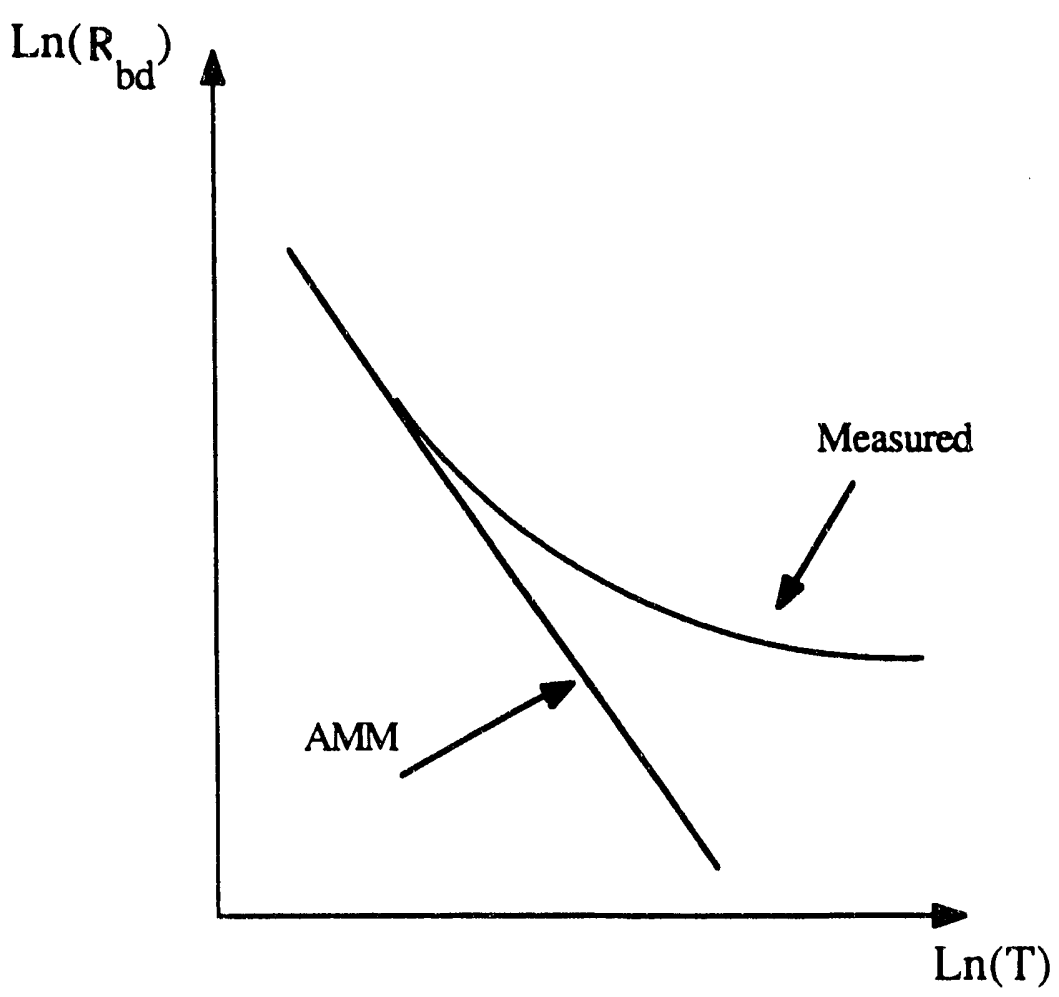

a)

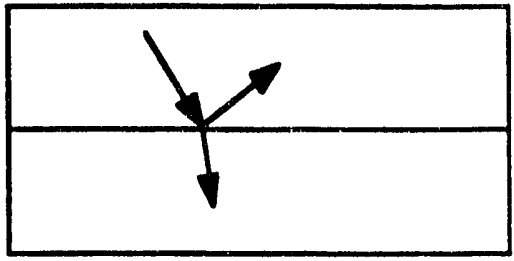

$\mathrm{T}<30 \mathrm{~K}$

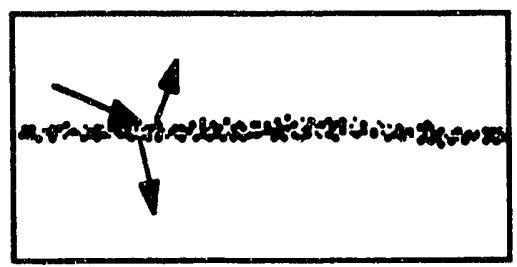

$\mathrm{T}>30 \mathrm{~K}$

b)

Fig. 4.2 a) Typical temperature dependence of the thermal boundary resistance. At temperatures $<30 \mathrm{~K}$, the thermal boundary resistance is proportional to $\mathrm{T}^{-3}$, for higher temperatures $R_{b d}$ approaches a constant value. b) At low temperatures, phonons propagate across the interface in much the same way as light reflects and refracts at the interface between two materials with different dielectric constants. At higher temperatures, the contact region is no longer a perfect interface, and additional scattering mechanisms become important. 


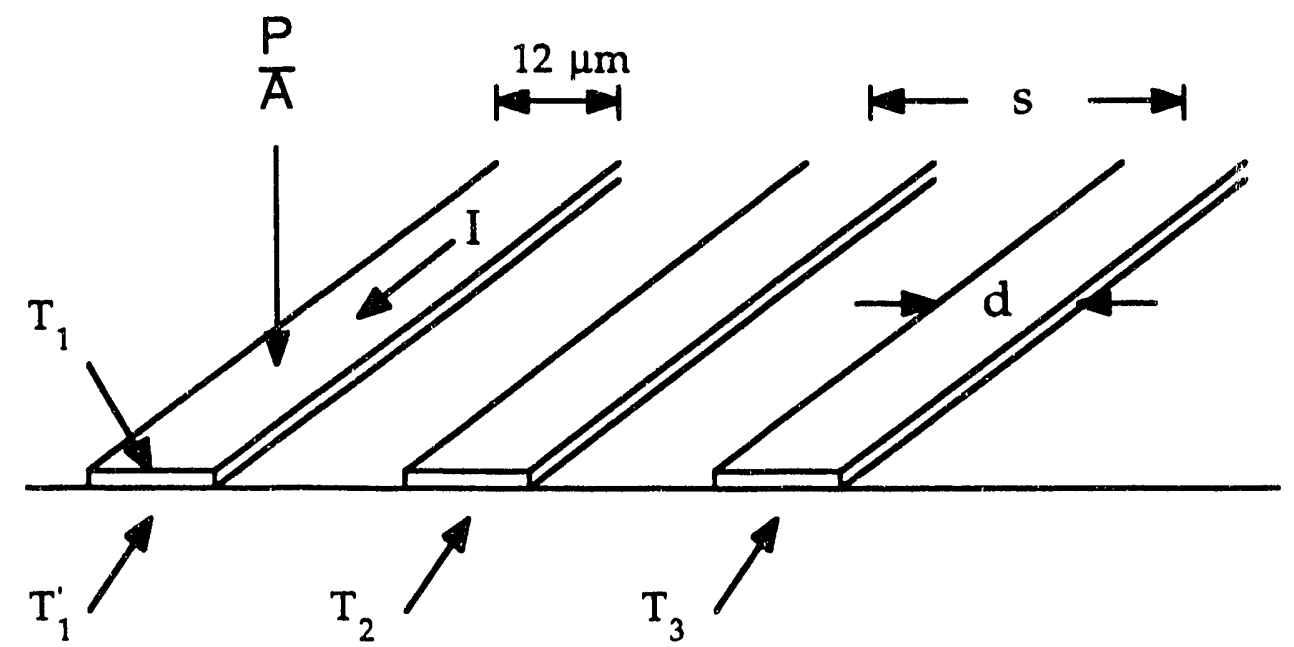

Fig. 4.3 Experimental configuration. The interface studied lies beneath the left strip, which is biased with a relatively high current $I$. 42 
closely spaced, long, narrow strips of YBCO which were patterned using standard photolithographic techniques. A silver fan-out pattern at each end of the strips led to current and potential pads. The dc electrical resistance of each strip was measured as a function of temperature from $90 \mathrm{~K}$ to $200 \mathrm{~K}$ using low bias currents for all three strips. Subsequently, a relatively large bias current was passed through strip 1 causing its temperature $T_{1}$ to rise significantly. The resistances of strips 2 and 3 were again measured with a small enough bias that the temperatures $T_{2}$ and $T_{3}$ deduced for each strip were essentially equal to the temperatures of the substrate directly underneath the strips. Both $\mathrm{T}_{1}$ and the dissipated power $P$ were measured. The temperature $T_{1}{ }^{\prime}$ of the substrate beneath strip 1 was calculated from a two dimensional solution of the time independent heat equation which is a good approximation to the experimental geometry. ${ }^{52}$

$$
T_{1}{ }^{\prime}=T+P(2 L \pi K)^{-1}\left[(x-1)^{2} \ln (x-1)+(x+1)^{2} \ln (x+1)-2 x^{2} \ln x\right] .
$$

Here $\mathrm{P} / \mathrm{L}$ is the power per unit length dissipated in strip $1, \mathrm{~T}$ is the temperature of the substrate under one of the other strips, $K$ is the thermal conductivity of the substrate, and $x$ is the ratio of the strip separation $s$ to strip width $d$. Both $T_{1}^{\prime}$ and $\kappa$ were deduced by using Eq. 4.7 for the two cases $T=T_{2}, x=s / d$ and $T=T_{3}, x=2 s / d$ as is shown in Fig. 4.3. The boundary resistance was then obtained from Eq. 4.1, where $\Delta \mathrm{T}=\mathrm{T}_{1}-\mathrm{T}_{1}{ }^{\prime}$. This measurement scheme is able to eliminate many of the experimental difficulties discussed earlier by reducing the contact area between the films and the substrate to several square microns, and the distance between adjacent thermometers to several microns.

\subsection{Sample Fabrication}

We chose to measure the thermal boundary resistance for several commonly used substrates. We were also interested in studying the effects of buffer layers on $R_{b d}$. The hope was that these buffer layers, which would be deposited between the YBCO film and 
the substrate, might be able to enhance $R_{b d}$, and thus permit the development of microbolometers with higher sensitivities. An additional collaboration was established with Conductus Inc. in which the samples would be deposited at Conductus, and subsequently patterned and measured at Berkeley. Epitaxial buffer layers which consisted of 100-500 $\AA$ of $\mathrm{SrTiO}_{3}, \mathrm{LaAlO}_{3}, \mathrm{CaTiO}_{3}$, or $\mathrm{MgO}$, were deposited by laser ablation on (1102) sapphire substrates after which $\approx 2000 \AA$ epitaxial films of YBCO were deposited in situ by laser ablation using deposition parameters that have been discussed elsewhere. ${ }^{53}$ In addition, YBCO films were deposited on (100) lanthanum aluminate substrates by the same method but without buffer layers. The films were subsequently ion milled in another chamber to remove $\approx 500 \AA$ of the YBCO surface and $\approx 2500 \AA$ of silver was ion beam sputtered in situ. The contact pads were patterned using standard photolithography and the silver was etched in a solution of $1 \mathrm{H}_{2} \mathrm{O}_{2}: 1 \mathrm{NH}_{4} \mathrm{OH}: 2 \mathrm{CH}_{3} \mathrm{OH}$. The YBCO strips were then similarly defined and etched in $\approx 0.5 \%$ phosphoric acid and oxygen annealed at $500{ }^{\circ} \mathrm{C}$ for 5 minutes. The resulting structure consisted of three strips, each $12 \mu \mathrm{m}$ wide, $300 \mu \mathrm{m}$ long and separated by $24 \mu \mathrm{m}$. Typical resistances above the resistive transition were $\approx 250 \Omega$, and the temperature coefficient of resistance was $d R / d T \approx 2 \Omega / K$. The transition widths of several Kelvin were not broadened by patterning indicating that the film quality was not significantly affected by the patterning process. The substrate was mounted on a computercontrolled temperature regulated stage.

\subsection{Experimental Results}

The major difficulties of the experiment were due to the dc nature of the measurements. Thermoelectric voltage offsets and slow drifts can often complicate dc measurements. In addition, we were concerned that the resistance of the YBCO strips might dependent on the bias current. It should be mentioned that these effects pertain only to strip 1, because the bias current of strips 2 and 3 was not varied. The simplest solution to the thermoelectric voltage problem was to reverse the current polarity. Also, by 
repeating the measurements for different values of bias current through strip 1 , we verified that its resistance was ohmic. An additional consideration was pointed out by Kenneth $\mathrm{E}$. Goodson.$^{54} \mathrm{He}$ argued that because the thermal conductivity of YBCO is small $\quad(\approx 0.01$ $\mathrm{W} / \mathrm{cm}-\mathrm{K}$ at $100 \mathrm{~K}),{ }^{55}$ temperature gradients can exist along the direction perpendicular to the plane of the film. The film closer to the interface is at a lower temperature than the section of the film furthest from the interface. Thus, the average temperature of the film, which is deduced from the resistance of the film, must be corrected for this effect. The analysis is rather simple and will not be presented here. It does imply however, that the thermal boundary resistance is $\approx 33 \%$ smaller than that calculated from the isothermal film model.

In a typical experiment, approximately $50 \mathrm{~mW}$ of power was dissipated in the first strip. The temperature rise of the strip relative to the substrate was $\approx 1.4 \mathrm{~K}$, whereas the temperature difference between strip 1 and 2 varied between $0.2 \mathrm{~K}$ and $2.0 \mathrm{~K}$. In Fig. 4.4 we show the measured temperature dependence of $\mathbf{R}_{b d}$ for the various samples. Also shown is the prediction of the acoustic mismatch model for YBCO on sapphire, neglecting the effect of the buffer layers. The thermal boundary resistances of samples a) and b), which had two buffer layers on sapphire, are weakly dependent on temperature while sample c), which had only one buffer layer on sapphire has a smaller $R_{b d}$ which decreases slightly with increasing temperature. The thermal boundary resistance of sample d), which had a lanthanum aluminate substrate without buffer layers, is similar in value to the previous samples, implying that the buffer layers have only a modest effect on $\mathbf{R}_{\mathrm{bd}}$. The larger scatter in the $R_{b d}$ data for sample d) is due to the lower thermal conductivity of lanthanum aluminate compared to sapphire.

For our samples the measured values of $R_{b d}$ varied from $0.3 \times 10^{-3}$ to $0.5 \times 10^{-3}$ $\mathrm{Kcm}^{2} / \mathrm{W}$, which is almost a factor of 50 larger than the prediction of the acoustic mismatch model at $100 \mathrm{~K}$. The relative insensitivity of $\mathbf{R}_{\mathrm{bd}}$ to temperature is a common observation in the high temperature regime, ${ }^{51}$ where the boundary region is thick 


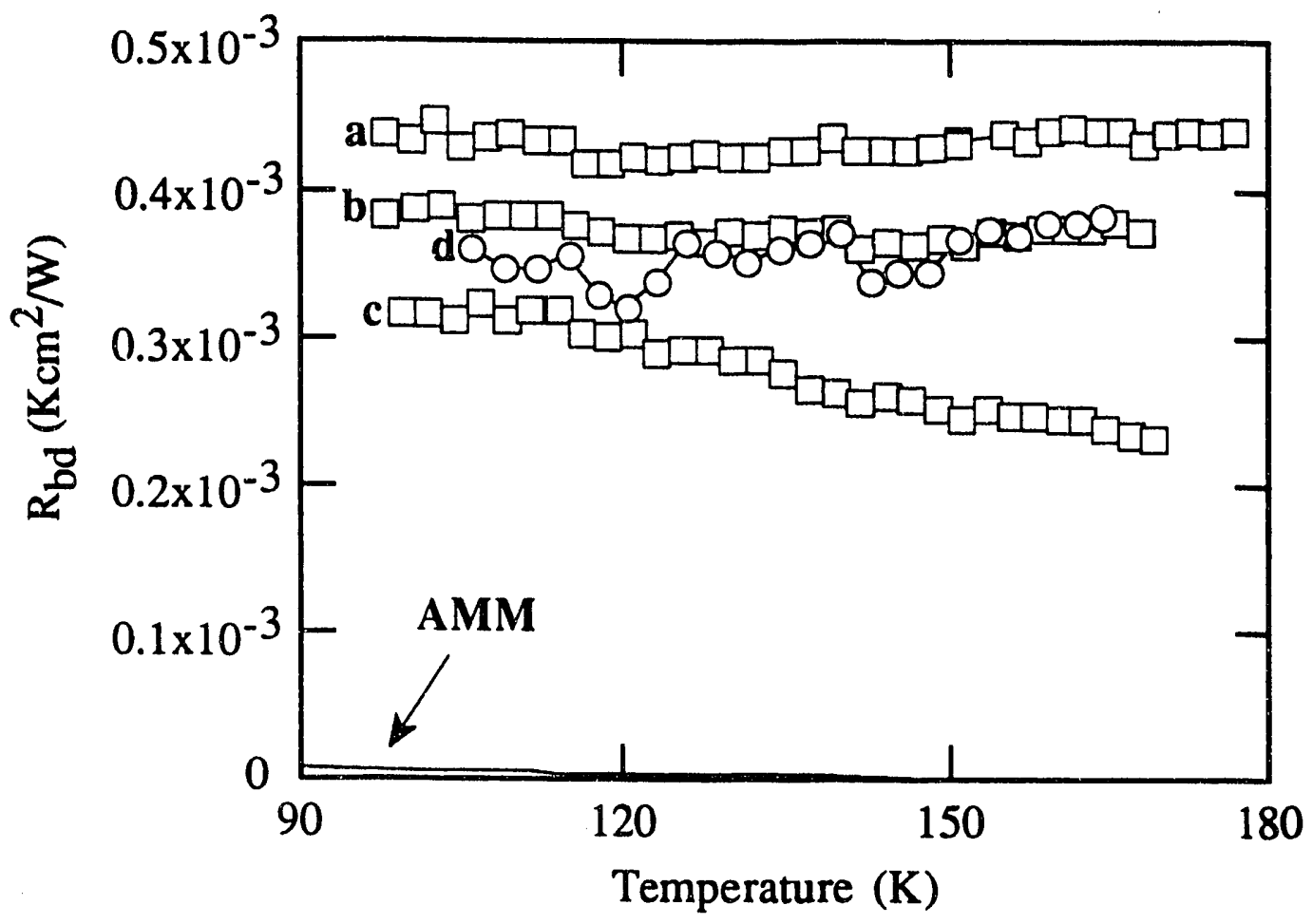

Fig. 4.4 Thermal boundary resistance for thin films of YBCO on sapphire with, a) $100 \AA \mathrm{SrTiO}_{3} / 100 \AA \mathrm{MgO}$, b) $200 \AA \mathrm{LaAlO}_{3} / 100 \AA \mathrm{CaTiO}_{3}$, and c) $500 \AA \mathrm{CaTiO}_{3}$ buffer layers. The solid line is the prediction of the acoustic mismatch model (AMM) for YBCO on sapphire without buffer layers. The thermal boundary resistance for YBCO on lanthanum aluminate is shown in d). 
compared to characteristic phonon wavelengths. The actual value of $R_{b d}$ depends on the surface quality of the interfaces, as well as the specific details of the deposition. Transmission electron micrographs of these films are not available, but those for similar high quality epitaxial films show a variety of kinds of disorder at the interface which propagate $\approx 100 \AA$ into the film. In addition, disordered layers of order $10 \AA$ thick are frequently seen at interfaces. ${ }^{56}$ The thermal conductivity of this layer is not known, but would have to be of order $\approx 10^{-4} \mathrm{~W} / \mathrm{cmK}$ to account for $\mathrm{R}_{\mathrm{bd}}$. Typical thermal conductivities of polycrystalline $\mathrm{YBCO}$ are $\approx 3 \times 10^{-2} \mathrm{~W} / \mathrm{cmK}$ at $100 \mathrm{~K},{ }^{57}$ which are too high to account for $\mathbf{R}_{\mathbf{b d}}$. This implies the existence of additional scattering mechanisms, such as damage in the substrate near the interface. Carr et al. measured the response of epitaxial films of YBCO on $\mathrm{MgO}$ to pulsed, broadband infrared radiation, as a function of film thickness. They inferred a value of $\mathrm{R}_{\mathrm{bd}} \approx 1.1 \times 10^{-3} \mathrm{Kcm}^{2} / \mathrm{W}$ at $90 \mathrm{~K}$, which is close to our measured range. Marshal et al. inferred a value of $R_{b d}=2.5 \times 10^{-3} \mathrm{Kcm}^{2} / \mathrm{W}$ for epitaxial films of $\mathrm{YBCO}$ on $\mathrm{MgO}$ at $300 \mathrm{~K}$ using the optical-transient grating method. ${ }^{58}$

I now consider whether the measured values for $\mathrm{R}_{\mathrm{bd}}$ are able to account for the observed nanosecond response time. The thermal time constant, $\tau=C / G$ resulting from the heat capacity $C$ of the film and the boundary conductance $G=A / R_{b d}$ for a film of area $A$, is several nanoseconds for a $500 \AA$ thick film and for our measured values of $R_{b d}$. The most thorough thermal analysis of these experiment is that of Flick et al., ${ }^{46}$ who analyzed the data of Frenkel et al. ${ }^{7}$ In the absence of specific data they modeled $R_{b d}$ with the acoustic mismatch model using $B=40 \mathrm{~K}^{4} \mathrm{~cm}^{2} / \mathrm{W}$. They concluded that the temperature profile through the film is essentially flat even for pulses as short as $150 \mathrm{ps}$, and that most of the temperature drop occurs across the film/substrate interface rather than through the substrate. These conclusions are still valid for the larger values of $\mathbf{R}_{\mathbf{b d}}$ that we measure. Realizing that the acoustic mismatch model underestimates $R_{b d}$ above $\approx 60 \mathrm{~K}$, Flick et al. calculate the effect of replacing it by a constant value of $0.2 \times 10^{-3} \mathrm{Kcm}^{2} / \mathrm{W}$, which was measured for a $\mathrm{Rh}: \mathrm{Fe} /$ sapphire interface. ${ }^{51}$ While the overall agreement between the 
calculations and the data was improved, the observed response could not be explained by the thermal hypothesis. Hence they concluded that the discrepancy is due to a nonbolometric mechanism. The values of $R_{b d}$ that we have measured are a factor $\approx 3.5$ larger than the largest values assumed by Flick et al. Consequently, the agreement between the analysis and the data will improve, thus weakening the evidence for a nonbolometric response on the ns time scale.

\subsection{Conclusions}

We have measured the thermal boundary resistance for YBCO thin films deposited on a variety of substrates. The magnitude of the thermal boundary resistance was almost two orders of magnitude higher than the predictions of the acoustic mismatch model, and is due perhaps, to a damage in the substrate close to the interface. The thermal relaxation time resulting from the heat capacity of the film and the boundary conductance is several ns for a typical film thickness. This result probably accounts for many of the experimental observation that were explained in terms of nonbolometric detection mechanisms.

The various response times that are observed should be understood in the context of a hierarchy of relaxation processes which can take place after a sudden deposition of energy in a high- $T_{c}$ film. Although many details of these processes are not clear, the following phenomena probably occur. In general, hot quasiparticles relax to large numbers of "gap

edge" quasiparticles and phonons of comparable energy in hundreds of femtoseconds. 59,60 "Gap edge" quasiparticles cannot recombine until energy leaves the excited region. Depending on the size of this region, the energy can dissipate in picoseconds by diffusion of quasiparticles and phonons through the film or, as shown in this work, phonons can carry energy across the boundary in nanoseconds. Heat spreads out into the substrate in microseconds, as in the case of the high- $T_{c}$ microbolometer ${ }^{5}$ and into the heat sink in milliseconds, as in the case of a conventional composite bolometer. The complexity of these essentially thermal processes is a serious impediment to the unambiguous 
identification of non-thermal detection mechanisms. 


\section{Chapter 5:_Low Temperature Superconducting_Microbolometers}

\subsection{Introduction}

Having gained an understanding of antenna-coupled bolometers and of thermal boundary resistances, we next inquired whether a low temperature version of the high- $T_{c}$ microbolometer could be a useful device. Potential applications of such a device can be understood by reference to applications of conventional composite bolometers operated at temperatures $\leqslant 4.2 \mathrm{~K}$. Unlike a conventional bolometer which accepts any throughput, the antenna coupled bolometer is limited to a single spatial mode with throughput equal to the square of the wavelength (section 2.6). Consequently its use is favored for applications to diffraction limited imaging or spectroscopy. Our estimates and measurements indicate that the antenna coupled bolometer can, in principle, achieve much higher sensitivities than conventional bolometers operated at the same temperature in applications where the background power loading is low. There are two NASA astrophysical spacecraft which are proposed for this decade and which require bolometers with very high sensitivities operating in low backgrounds. The Space Infrared Telescope Facility (SIRTF) will have a single bolometer for $\lambda \approx 1 \mathrm{~mm}$ and a four bolometer array for shorter wavelengths, all operate at $100 \mathrm{mK}$. Backgrounds will be very low because the optics will be cooled to $\approx 1.5 \mathrm{~K}$. The Submillimeter Moderate Mission $\left(\mathrm{SM}^{3}\right)$ which will have low emissivity $\approx 200 \mathrm{~K}$ optics will require large format bolometer arrays for diffraction limited imaging which will probably operate near $300 \mathrm{mK}$ as well as $100 \mathrm{mK}$ arrays for spectroscopy. In addition, bolometers are used for important astrophysical applications at millimeter and submillimeter wavelengths from ground based (mountain top or south pole), airborne, balloon, and sounding rocket platforms. Important scientific goals include observations of molecular line emission from interstellar gas and planetary atmospheres, continuum emission from interstellar dust, and observations of the cosmic background radiation.

For most applications, external filters are used to limit the detected bandpass. At 
submillimeter wavelengths, high frequency filter leakage is a major problem since the power from a thermal source detected by a conventional bolometer with constant throughput increases as the square of the frequency. For an antenna coupled bolometer, the single mode throughput decreases as the frequency squared, so that the spectrum of detected power is flat and the filtering task is easier. As will be discussed in section 5.6, the antennas can also be used in conjunction with lithographed filter and tuning elements. Thus, it may be possible to construct multiplexed systems by designing on-chip networks with different bandpass filters fed from a single antenna. Using well developed reproducible lithographic techniques it should then be possible to make powerful array receivers with multiple spectral bands.

In the following section, I will present the basic operating principles of the low- $T_{c}$ microbolometer and will make estimates of the expected performance. ${ }^{11,12}$ In section 5.3, I will describe the experimental apparatus and the device fabrication process. The experimental results will be presented in section 5.4. The low- $\mathrm{T}_{\mathrm{c}}$ microbolometer was successfully used to make the first direct measurements of the frequency dependent optical efficiency of log-periodic antennas up to $\approx 2 \mathrm{THz}$, these measurements will be discussed in section 5.5. A brief discussion of altemative coupling schemes will be presented in section 5.6.

\subsection{Operating Principles}

The basic principles of the low- $T_{c}$ microbolometer are very similar to the high- $T_{c}$ counterpart, a transition edge thermometer acts both as a resistive load and as a sensitive thermometer to thermalize the if power from an antenna and to measure the resultant temperature rise. As was previously discussed, the sensitivity of a thermal detector is ultimately limited by its thermal isolation from the environment. In the proposed geometry the absorbed power is dissipated via two paths. The first is direct heat flow from the superconducting thermometer into the dielectric substrate. At temperatures above $\approx 10 \mathrm{~K}$ 
the thermal conductance is dominated by the bulk spreading resistance arising from the thermal conductivity of the substrate, as is the case for the high- $T_{c}$ superconducting microbolometer that was described in chapter 3. At the lower temperatures of interest here, the thermal boundary resistance contributes significantly to the thermal isolation, and below several Kelvin controls the heat flow (chapter 4 contains a comprehensive discussion of thermal boundary resistances). The acoustic mismatch theory, developed by W. A. Little, ${ }^{47}$ has been very successful in explaining thermal boundary resistances at temperatures below $\approx 30 \mathrm{~K}$. According to this theory, the thermal conductance is given by

$$
\mathrm{G}=\mathrm{AT}^{3} / \mathrm{B}
$$

where $\mathrm{A}$ is the contact area, and $\mathrm{T}$ is the temperature. The parameter $\mathrm{B}$ depends on the densities and sound velocities of the materials and is usually $\approx 20 \mathrm{~K}^{4} \mathrm{~cm}^{2} / \mathrm{W}$.

The contact area between the metal absorber and the antenna terminals provides an alternate path for heat dissipation. In addition to the phonon relaxation mechanism discussed above, there exists the possibility that unpaired electrons in the superconducting thermometer, which is maintained at the center of its resistive transition, could also transfer energy across this interface. If however, the antenna is made from a superconductor whose $T_{c}$ is higher than the operating temperature of the device, then the Andreev reflection ${ }^{15}$ of electrons at the interface between the if absorber and the superconducting antenna traps the electrons and hence the absorbed energy in the active region. The transmission probability for quasiparticles in the metal absorber to cross the interface is proportional to $\exp (-\Delta / \mathrm{kT})$. For a superconductor whose energy gap $\Delta$ is much larger than the thermal energy of the electrons in the metal strip, the predicted transfer of energy will thus be negligible. A convenient choice is $\mathrm{Nb}$ with a transition temperature of $\approx 9.2 \mathrm{~K}$. The trapping of energy at the interface between a metal an a superconductor is the subject of chapter 7 . 
Another effect which may be of importance at very low temperatures $(\leq 1 \mathrm{~K})$ is the thermal resistance between the electrons and the lattice in the thermometer. At such low temperatures the energy transfer between the electrons and phonons is less than perfect because there are a finite number of electrons attempting to transfer energy to phonons with a low occupation probability. The result is that the normal electrons in the superconducting thermometer absorb radiation and are heated above the lattice temperature. The thermal resistance between the electrons and phonons has been measured in copper with the result that $\mathrm{G}=5 \times 10^{3} \mathrm{VT}^{4} \mathrm{~W} / \mathrm{K}$, where $\mathrm{V}$ is the volume in $\mathrm{cm}^{3}{ }^{61}$ This effect can dominate the thermal boundary resistance at low enough temperatures for small film thicknesses ( for a $1000 \AA \mathrm{Cu}$ film the effects are comparable at $\approx 1 \mathrm{~K}$ ). To the best of my knowledge, electron heating effects in superconductors that are biased at the center of the resistive transition have not been investigated. Furthermore, this effect may be less important for superconductors which have a stronger electron phonon interaction than most metals. Estimates of this effect thus, will not be included in the design calculations.

As discussed in chapter 2, the figures of merit that are commonly used to characterize bolometers are the voltage responsivity $S$, the time constant $\tau$, and the noise equivalent power NEP. The voltage responsivity is given by equation (2.3),

$$
S=\frac{I d R / d T}{G(1+i \omega \tau)}
$$

where $I$ is the bias current, $d R / d T$ is the temperature coefficient of resistance, $\tau=C / G$, and $\omega=2 \pi f$ is the modulation frequency. To avoid thermal runaway due to self bias heating, ${ }^{62}$ the bias current must satisfy

$$
\alpha \equiv \frac{\mathrm{I}^{2}(\mathrm{dR} / \mathrm{dT})}{\mathrm{G}}<1
$$

For design purposes it is customary to pick a nominal value of $\alpha=0.3$. The NEP is given 
by the usual sum of phonon, Johnson, amplifier, and $1 / \mathrm{f}$ noise contributions. If the transition width is a fraction $\beta$ of the operating temperature then, neglecting amplifier and 1/f noise,

$$
\mathrm{NEP}=\frac{1}{\eta} \sqrt{4 k T^{5} \frac{A}{B}\left(1+\frac{\beta}{2 \alpha}\right)}
$$

Estimates of the Johnson noise and phonon noise contributions to the NEP as well as the total NEP, all as a function of operating temperature (neglecting amplifier noise) are plotted in Fig. 5.1. The heat flow is assumed to be limited by the thermal boundary resistance for a total contact area, including contact with the antenna edges, $A=2 \times 2 \mu \mathrm{m}^{2}$. The optical efficiency is set at $50 \%$, and $\beta=0.1$. The time constant is calculated from $\tau=C / G$ where $C$ is the total heat capacity of the thermometer. At temperatures of interest $(T<4 \mathrm{~K})$, the electronic contribution to the heat capacity ( $\mathrm{C}$ proportional to $\mathrm{T}$ ) dominates the lattice contribution ( $\mathrm{C}$ proportional to $\mathrm{T}^{3}$ ), thus $\tau$ will be proportional $\mathrm{T}^{-2}$. A crude estimate is $\tau \approx 10^{-6} \mathrm{~s}$ at $100 \mathrm{mK}$. These calculated values of NEP are significantly lower than are obtained for the more conventional bolometer architectures. For example, the estimated NEP at $100 \mathrm{mK}$ is $3 \times 10^{-18} \mathrm{WHz}^{-1 / 2}$ for the antenna coupled bolometer, compared with $3 \times 10^{-17} \mathrm{WHz}^{-1 / 2}$ for the more conventional bolometers. ${ }^{63}$ The effect could be large enough to permit operation at $300 \mathrm{mK}$, where $100 \mathrm{mK}$ would otherwise be required.

The rf impedance of the strip, when deposited directly between the antenna terminals is given by Eq. 3.6, $\mathrm{Z}_{\text {bolo }}=\mathrm{Z}_{\mathrm{L}}+\mathrm{Z}_{\mathrm{S}} / w$. The first term is due to the geometrical inductance of the strip while the second term is due to its surface resistance. The two-fluid model that was used to model the if impedance of the $\mathrm{YBCO}$ strip discussed in chapter 3, is no longer strictly valid for the BCS-like, low- $T_{c}$ superconductors of interest here. This model should, however, provide a good description far enough below $T_{c}$ that pair breaking is not important, but where kinetic inductance effects must be considered. A complete understanding of $Z_{S}$ at the midpoint of the transition for higher frequencies would 


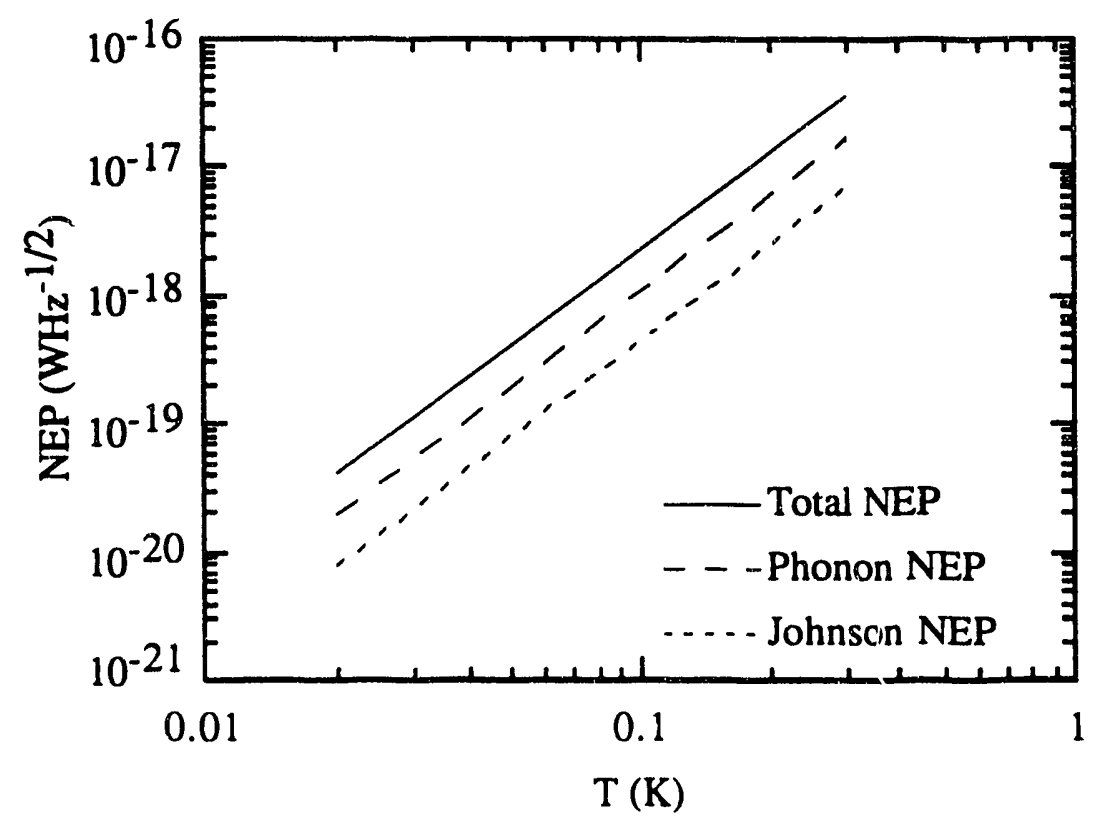

Fig. 5.1 Estimates of the Johnson, phonon and total NEP as a function of operating temperature. We assume the heat flow to be limited by the thermal boundary resistance from a $2 \times 2 \mu \mathrm{m}$ square contact area and pick $B=20$ $\mathrm{K}^{4} \mathrm{~cm}^{2} / \mathrm{W}$. The optical efficiency is assumed to be $50 \%$, the stability factor 0.3 and the superconducting transition width $10 \%$ of the operating temperature. Under these conditions the sensitivity is limited by the phonon noise. 
require a full Mattis-Bardeen calculation ${ }^{64}$ including pair breaking for an inhomogeneous superconductor with finite transition width. In practice, it should be sufficiently accurate to use a value that is close to the normal state resistance per square of the film.

\subsection{Experimental Apparatus and Device Fabrication}

In order to measure the electrical and optical properties of a variety of devices, a dedicated test cryostat was designed and constructed. A home-made, self-contained ${ }^{3} \mathrm{He}$ refrigerator with a minimum temperature of $260 \mathrm{mK}$ was constructed. ${ }^{65}$ A temperature regulated stage was used in conjunction with a commercial PID controller, ${ }^{66}$ which resulted in a temperature stability better than $0.01 \mathrm{mK}$ at $300 \mathrm{mK}$, and $\approx 0.5 \mathrm{mK}$ at $4.2 \mathrm{~K}$. A lead shielded, ${ }^{4} \mathrm{He}$ cooled transformer was used as a low noise amplifier. I also designed and assembled an optical system to measure the efficiency of planar lithographed antennas over a specified frequency band. It consisted of a variable temperature blackbody with narrow bandpass filters, a tuning fork chopper ${ }^{67}$ to modulate the radiation from the blackbody, and an off-axis ellipsoidal mirror to focus this radiation onto a quartz hemispherical lens on which the detector is mounted. In addition, the system was designed to accommodate measurements with a Fourier transform infrared spectrometer (FTIR). The optical system will be discussed in detail in section 5.4 .

A collaborative effort was established with Professor D. E. Prober's group at Yale University in which the devices would be fabricated at Yale, and subsequently tested at Berkeley. We investigated a variety of superconductors with nominal transition temperatures between $300 \mathrm{mK}$ and $4.2 \mathrm{~K}$, for the transition edge thermometer. Table 5.1 lists the deposition parameters for titanium, aluminum, and the $\beta$ and bcc phases of tantalum. Also listed are the deposition parameters for niobium $\left(T_{c} \approx 9.2 \mathrm{~K}\right)$, which was used for the antenna. All of the devices were deposited on 0.008 " thick fused quartz wafers. The microbolometers were fabricated by standard photolithographic techniques. The transition edge thermometers were patternec by liftoff into $2 \mu \mathrm{m}$ wide strips, and 


\begin{tabular}{|c|c|c|c|c|}
\hline Material & Deposition Method & Deposition Rate & Thickness & Resistivity \\
\hline Titanium & e-beam evaporation & - & $425 \AA$ & $58 \mu \Omega$-cm \\
\hline Aluminum & rf sputtering & $6.5 \AA / \mathrm{s}$ & $400 \AA$ & $3 \mu \Omega$-cm \\
\hline$\beta$-Tantalum & $\mathrm{dc}$ sputtering & $4 \AA / \mathrm{s}$ & $750 \AA$ & $146 \mu \Omega$-cm \\
\hline bcc-Tantalum & $\mathrm{dc}$ sputtering & $40 \AA / \mathrm{s}$ & $500 \AA(30 \AA \mathrm{Nb})$ & $27 \mu \Omega$-cm \\
\hline Niobium & $\mathrm{dc}$ sputtering & $12 \AA / \mathrm{s}$ & $1000-2000 \AA$ & - \\
\hline
\end{tabular}

Table 5.1. Material deposition parameters. 
varied in length between $4-10 \mu \mathrm{m}$, the thickness of the films was $400-800 \AA$. The edges of the strips were ion milled to clean their top surface, after which $\approx 2000 \AA \mathrm{Nb}$ was sputtered and patterned by liftoff to define the antenna. A schematic of the active region is shown in Fig. 5.2.

The following is a summary of the $R(T)$ characteristics of the thermometer materials:

a) Titanium: The transition temperature of the bulk occurs at $\approx 390 \mathrm{mK} .{ }^{49}$ The film properties on the other hand, are very sensitive to the particular deposition method. Many deposition attempts using both sputtering and conventional evaporation techniques proved unsuccessful. HYPRES Inc. was kind enough to supply us with high-quality, e-beam evaporated, titanium films. These films had superconducting transition temperatures of $525 \mathrm{mK}$, and transitions widih of $\approx 7 \mathrm{mK}$. Unfortunately, the films did not maintain these useful characteristics subsequent to patterning of the microbolometer structures. The reasons for this are not yet clear, but may be related to the fact that titanium is very reactive, and thus may be affected by standard processing techniques. We are currently trying to optimize the deposition and fabrication process for these films.

b) Aluminum: Aluminum is one of the easiest metals to deposit and pattern, and has been extensively characterized. It's only disadvantage, for our application, is that it has a low electrical resistivity of $\approx 3 \mu \Omega-\mathrm{cm}$, which makes matching of the strip and antenna impedances difficult. The $R(T)$ characteristics of a typical aluminum microbolometer is shown in Fig. 5.3a.

c) B-Tantalum.: Tantalum films occur in two phases. One such phase, commonly referred to as $\beta$-Ta, has been reported to superconduct near $500 \mathrm{mK}$. Reliable references on this phase however, are difficult to find. ${ }^{68}$ Since this phase is considered to be the 'bad' phase of $\mathrm{Ta}$, not much effort has been invested in optimizing its deposition parameters. The films are usually sputter deposited on room temperature substrates. ${ }^{69}$ The resistance versus temperature characteristics of a typical film is shown in Fig. 5.3b. The resistive transition is wide and occurs at $\approx 2.5 \mathrm{~K}$, indicative of the existence of a mixed phase. It is 


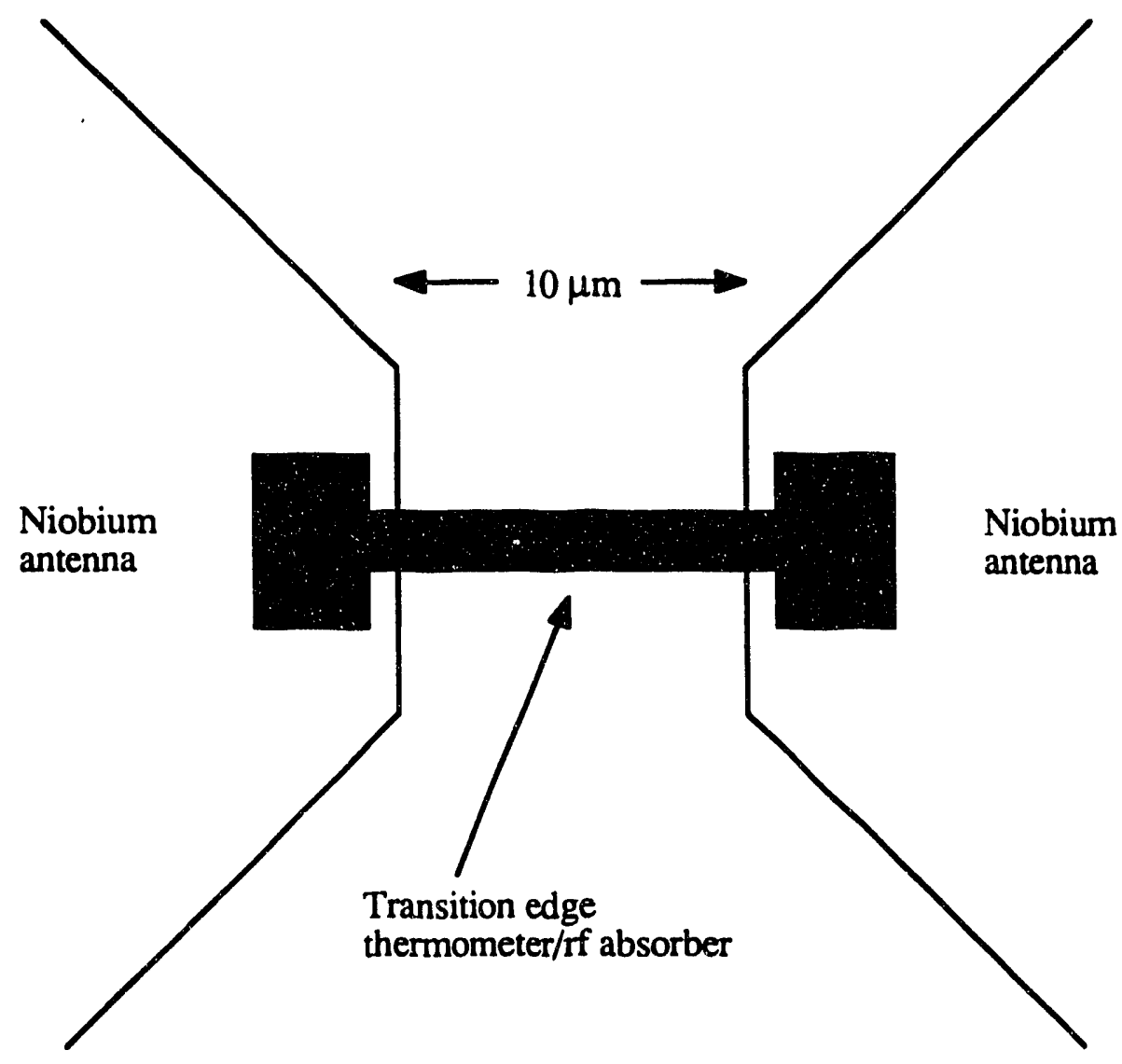

Fig. 5.2 Close-up view of the active region. The width of the strip is $2 \mu \mathrm{m}$, while its length varies between $2-10 \mu \mathrm{m}$. The large contact pads at the ends of the strip ensure good electrical contact between the antenna and the active region. For calculation purposes, the contact area of the strip with the antenna is assumed to be $\approx 2 \times 4 \mu \mathrm{m}^{2}$. 
clear that the deposition parameters are not optimal, and will require further investigation. This will be carried out at Yale University in the next 1-2 years.

d) bec-Tantalum: The deposition of this phase is a well known technology, it is nucleated by either depositing the films on a hot substrate $\left(\approx 700^{\circ} \mathrm{C}\right)$ or on top of a thin niobium buffer layer. ${ }^{69}$ The $R(T)$ characteristics of a bcc-Ta microbolometer of the latter variety, is shown in Fig. 5.3c. The resistive transition is narrow and occurs at $4.2 \mathrm{~K}$.

\subsection{Electrical Characterization}

The microbolometers were tested by measuring $R(T)$, load curves, and voltage noise. The current source for all of the measurements consisted of a cold $(4.2 \mathrm{~K})$ load resistor, a $9 \mathrm{~V}$ battery, and a variable voltage divider. All other commercial current sources either had excessive $60 \mathrm{~Hz}$ line pickup, or produced electrical transients which subsequently resulted in an open circuit.

The following is a summary of the measured electrical and thermal properties of the devices:

a) bcc-Ta: The I-V characteristic of a typical device is shown in Fig. 5.4a. The resultant load curve (resistance $R$ versus bias power $P$ ) is shown in Fig. 5.4b. The linear dependence of $\mathrm{R}$ on $\mathrm{P}$ for low powers, suggests that the effects of non-thermal nonlinearities are small. The measured thermal conductance, determined from the slope of Fig. 5.4b and from the temperature coefficient of resistance $\mathrm{dR} / \mathrm{dT}$, is $\approx 1.3 \times 10^{-7} \mathrm{~W} / \mathrm{K}$. This should be compared to the predicted value of $G=5.8 \times 10^{-7} \mathrm{~W} / \mathrm{K}$, resulting from the thermal boundary resistance between the active region and the substrate. From Eq. 5.3, the maximum bias current that can be applied without causing thermal runaway is $\approx 17 \mu \mathrm{A}$, the corresponding voltage responsivity is then $S=1.7 \times 10^{4} \mathrm{~V} / \mathrm{W}$.

The voltage noise was measured using a circuit similar to that in Fig. 3.4. The dependence of the voltage noise on the resistance of the microbolometer, for a bias current of $20 \mu \mathrm{A}$, is shown in Fig. 5.5. A large narrow peak in the voltage noise is observed just 


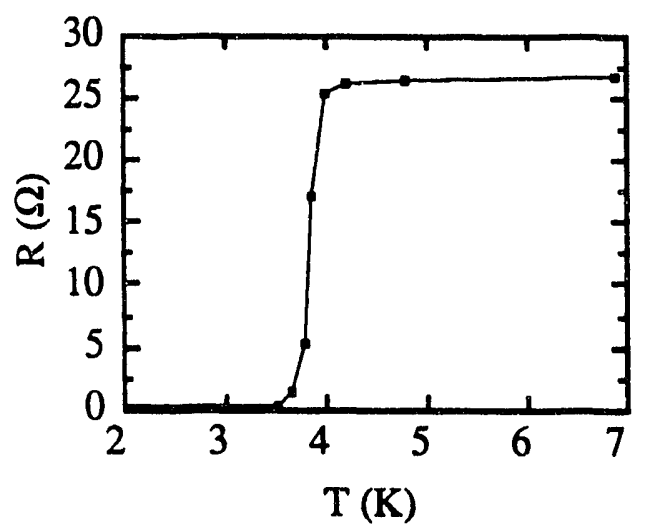

a)

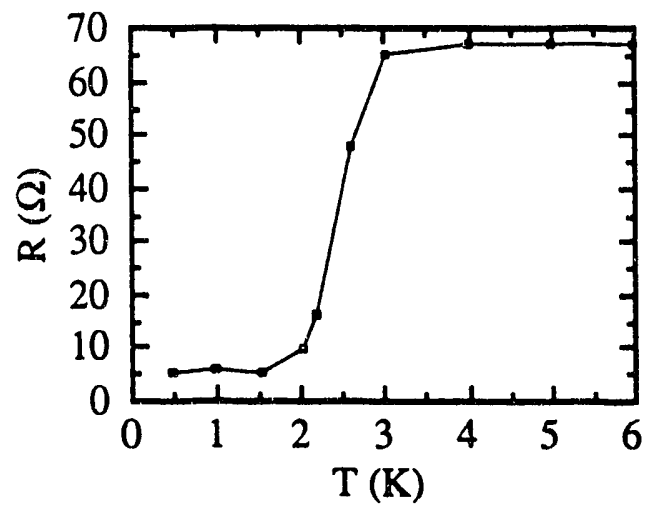

b)

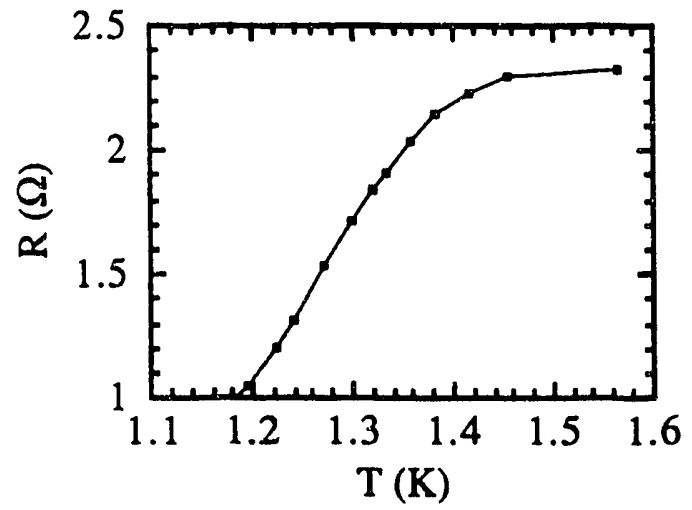

c)

Fig. 5.3 Resistance versus temperature characteristics for a) bcc-Ta, b) $\beta$-tantalum, and c) aluminum microbolometers. The residual resistance at the foot of the transition is due to contact resistance between the antenna and the electrical leads. 


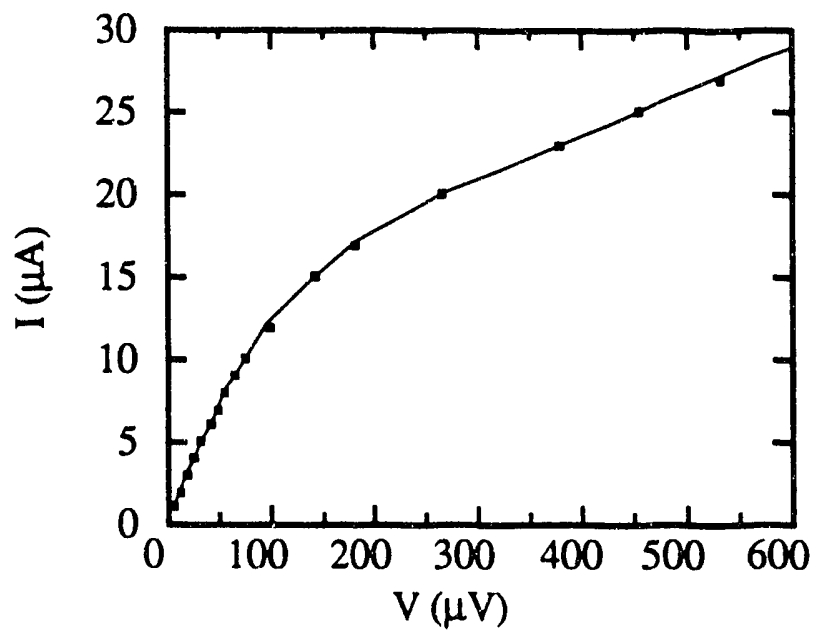

a)

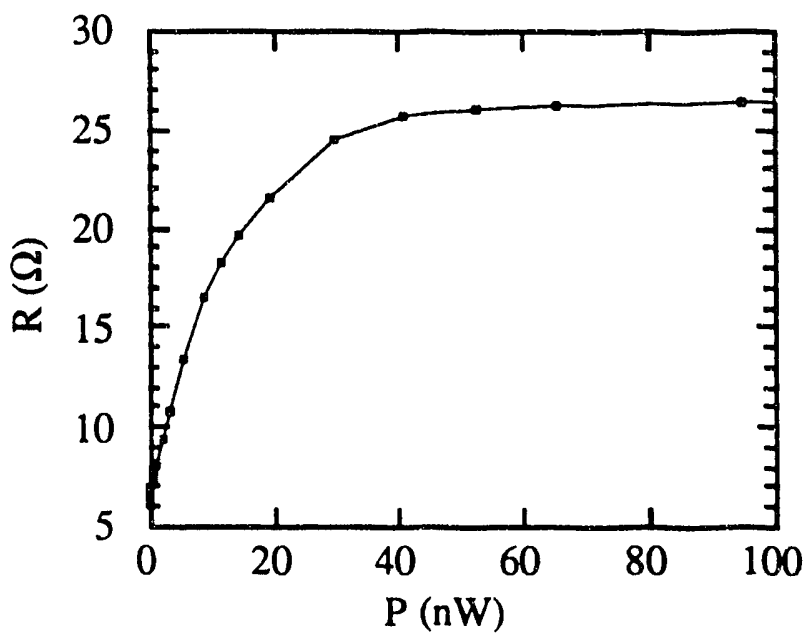

b)

Fig. $5.4 \quad$ a) Current-voltage characteristics for a typical bcc-Ta microbolometer. b) Resistance versus bias power load curve derived from a). The voltage responsivity is obtained from the slope of the curve for bias powers $\leq 20 \mathrm{nW}$. For higher powers, the thermometer is driven normal. 


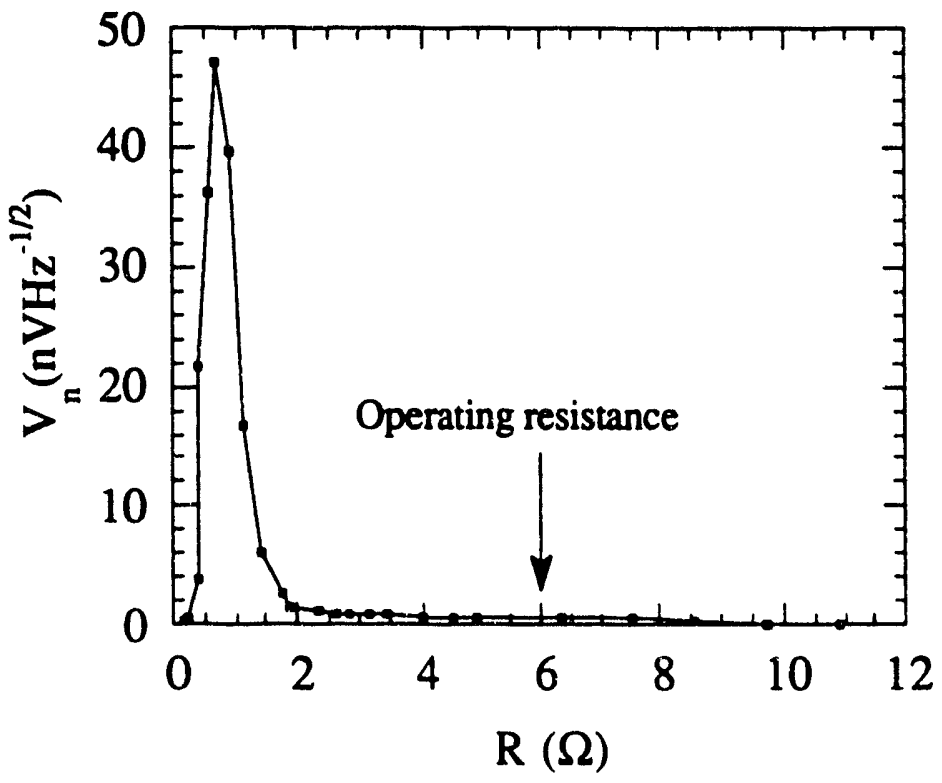

Fig. 5.5 Voltage noise of a current biased bcc-Ta microbolometer. A narrow noise peak is commonly observed at the foot of the resistive transition. At the operating resistance however, the noise is small, and is dominated by thermal fluctuations in the active region. This noise peak was not observed for other thermometer materials investigated, this suggests that this peak is not intrinsic to the device, but rather an artifact of the fabrication process. 
below the foot of the transition. The electrical NEP which is the ratio of the voltage noise and the voltage responsivity is $\approx 2 \times 10^{-14} \mathrm{WHz}^{-1 / 2}$ at the center of the resistive transition. This is $\approx 50 \%$ higher than the predicted phonon NEP. The difference may be due to residual noise from the noise peak. The observed peak in the voltage noise at the foot of the resistive transition is influence by the fabrication parameters, ${ }^{70}$ so is probably not intrinsic to the device.

b) $\beta$-tantalum: The thermal conductance that is deduced from the load curve is $=8 \times 10^{-8} \mathrm{~W} / \mathrm{K}$ which is close in value to the calculated $\mathrm{G}$ of $\approx 1.2 \times 10^{-7} \mathrm{~W} / \mathrm{K}$. In addition, excess voltage noise near the resistive transition was not observed. The resultant electrical NEP for this device was $\approx 1 \times 10^{-14} \mathrm{WHz}^{-1 / 2}$ which should be compared to the predicted NEP of $8 \times 10^{-15} \mathrm{WHz}^{-1 / 2}$. For this device, the NEP is not limited by phonon noise because of the wide resistive transition, which subsequently reduces the responsivity.

c) Aluminum: The thermal conductance that is deduced from the measured load curve is $\approx 2 \times 10^{-8} \mathrm{~W} / \mathrm{K}$, which is close to the calculated $\mathrm{G}=2 \times 10^{-8} \mathrm{~W} / \mathrm{K}$, thus providing additional confirmation of our predictions. The resistance of a typical thermometer strip is $\approx 1 \Omega$ at the center of the resistive transition, which is a very poor match to the antenna impedanie. Using e-beam lithography however, it should be possible to pattern narrow ( $\leq 1 \mu \mathrm{m})$ thermometer strips, which would improve the impedance match. The reduced area of the active region will also reduce the thermal conductance, thus increasing the sensitivity of the device. For example, the predicted NEP for a contact area $A=0.1 \times 10 \mu \mathrm{m}^{2}$ is $\approx 1.5 \times 10^{-16} \mathrm{WHz}^{-1 / 2}$ at $\mathrm{T}=1.2 \mathrm{~K}$, which is slightly higher than the sensitivity of conventional composite bolometers operating at $300 \mathrm{mK}^{71}$

The most important conclusion from these measurements is that the measured thermal conductance of three different transition edge thermometers, operating at different temperatures, agreed with the values predicted by the acoustic mismatch model. This is a very important result since it means that we understand and can successfully predict the thermal characteristics of the microbolometer. 


\subsection{Frequency Dependent Optical Efficiency of Log-Periodic Antennas}

Planar lithographed antennas are rapidly becoming the technology of choice in many applications requiring operation at high frequencies and array compatibility. A number of planar antennas have been suggested for use at submillimeter wavelengths including the log-periodic and log-spiral antennas, ${ }^{42,72,73}$ bow tie antennas, ${ }^{24}$ slot antennas, ${ }^{74}$ etc. These structures have been used to couple radiation to a variety of detectors including Superconductor-Insulator-Superconductor (SIS) direct detectors and mixers, ${ }^{31}$ bismuth microbolometers, ${ }^{27}$ and more recently the low and high- $\mathrm{T}_{\mathrm{c}}$ transition edge microbolometers discussed here. In addition, self-complementary antennas have been used for the propagation and detection of pulsed terahertz radiation. ${ }^{75}$ Despite their widespread use, there have been relatively few measurements of the frequency dependence and optical efficiency of the antenna, the primary reasons being due to the capacitive rolloff characteristic of SIS detectors and diodes at high frequencies, and to the relatively low sensitivity of microbolometers operated at $300 \mathrm{~K}$. In what follows I will discuss our measurements of the frequency dependent optical efficiency of log-periodic antennas from $90 \mathrm{GHz}$ to $2 \mathrm{THz}$ using a low- $\mathrm{T}_{\mathrm{c}}$ microbolometer, a Fourier transform infrared spectrometer (FTIR), and a blackbody reference.

The antenna investigated was a log-periodic antenna with a self-complementary structure shown in Fig. 5.6. Log-periodic antennas were initially invented to achieve broadband characteristics, but later used for their favorable radiation patterns. The geometry of the structure is such that the geometrical properties repeat themselves when the length scale is changed by a factor $\tau$. As a result, the radiation pattern and antenna impedance will be identical for all frequencies $v_{0}$ and $\tau^{-1} v_{0}{ }^{76}$ In addition, for a selfcomplementary structure, the antenna impedance (but not necessarily the radiation pattern) is frequency independent and given by $Z_{\text {ant }}=377[2(1+\varepsilon)]^{-1 / 2} \Omega$, where $\varepsilon$ is the dielectric constant of the substrate. ${ }^{24}$ When the antenna is deposited on quartz, $\mathrm{Z}_{\mathrm{ant} .} \approx 120 \Omega$. Since planar antennas deposited on a dielectric surface radiate primarily into the dielectric, the 


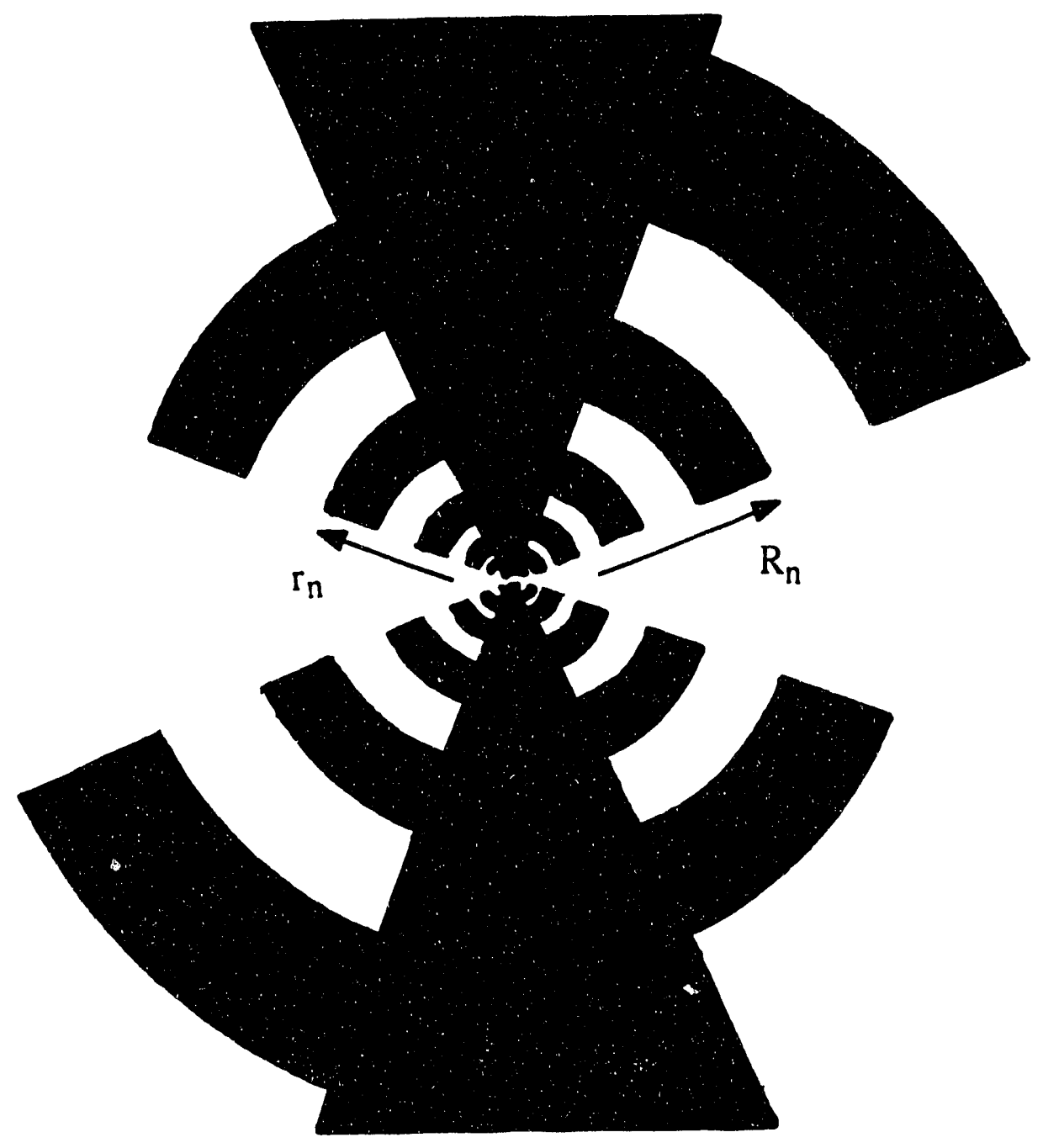

Fig. 5.6 Expanded view of a sheet circular log-periodic antenna. 
signals are introduced through the back surface of the dielectric, which is often placed on the back side of a dielectric lens. The specific type of log-periodic antenna that was used in the experiments is called a sheet circular tooth antenna. An expanded view is shown in Fig. 5.6. It consists of two sets of teeth. The radii of the arms which define the location of successive teeth are related by $\tau=R_{n+1} / R_{n}=r_{n+1} / r_{n}$. This same ratio defines the lengths and widths of successive teeth. The location of the second set of teeth $r_{n}$ is related to the first by the relation $r_{n}=\tau^{1 / 2} R_{n}$. It is because of this 'left-right' asymmetry of the structure, that the properties of the antenna are identical for all frequencies $v_{0}$ and $\tau^{-1 / 2} v_{0}$, rather than $\tau^{-1} v_{0}{ }^{76}$ The antenna had a scaling factor of $\tau=0.5$. The distance from the vertex of the antenna to the inner edge of the innermost tooth was $12 \mu \mathrm{m}$, and to the outer edge of the outermost tooth was $2920 \mu \mathrm{m}$, giving approximate coupling limits of $20 \mathrm{GHz}$ to $4 \mathrm{THz}$ (or $12.5 \mathrm{~mm}$ to $76 \mu \mathrm{m}$ ) for an otherwise lossless antenna. ${ }^{76}$

In order to measure the intrinsic frequency response of the antenna, it is necessary that the impedance of the sensor which terminates the antenna not vary significantly within the frequency range of interest, and in addition, the sensitivity of the device should be compatible with the measurement technique. The low- $\mathrm{T}_{\mathrm{c}}$ microbolometer satisfies both of these requirements. As discussed in section 5.2, the coupling of if currents into the strip will not depend on the of frequency even if the resistive component of $Z_{\text {bolo }}$ is much smaller than the antenna impedance. In addition, the NEP of $3 \times 10^{-14} \mathrm{WHz}^{-1 / 2}$ for the bccTa microbolometer is sufficiently low to detect signals from a FTIR spectrometer and a blackbody source. The low $-T_{c}$ microbolometer is thus very useful for probing the intrinsic properties of the antenna.

The devices were fabricated on fused quartz substrates using the process described in section 5.2. The substrate was then mounted onto the back side of a hemispherical crystal quartz lens with a thin layer of grease, and electrical contacts were made at the antenna terminals with silver paint. The substrate-lens combination was temperature regulated at the center of the resistive transition of the tantalum strip using a commercial germanium 
resistance thermometer ${ }^{38}$ and PID controller, ${ }^{66}$ the resulting temperature stability was better than $0.5 \mathrm{mK}$ at $4.2 \mathrm{~K}$. Because of the low impedances characteristic of antenna coupled devices, it was necessary to use a transformer as the first stage of amplification. A blocking capacitor $(\mathrm{C}=1000 \mu \mathrm{F})$ was used in conjunction with a PAR model 190 (10:1000 turn ratio) low noise transformer. ${ }^{77}$

The optical configuration for the FTIR measurements is shown in Fig. 5.7. An offaxis ellipsoidal mirror was used to collimate the beam onto the quartz lens (diameter $=1.3$ $\mathrm{cm}$ ), whose center coincides with one of the focal points. A light pipe was used to feed the radiation from the FTIR to the second focal point of the mirror. The power spectrum of the incident radiation was determined by replacing the microbolometer with a conventional composite bolometer operated at $\mathrm{T}=2.0 \mathrm{~K}$ whose absorbing element was a bismuth film. ${ }^{78}$ The properties of the bismuth film absorber are essentially independent of frequency over the range of our measurements. ${ }^{78}$ The frequency response of the microbolometer $R(v)$ was determined from the spectra measured with the microbolometer $S_{m}(v)$, and the composite bolometer $S_{b}(v)$ as follows,

$$
R(v)=\left[S_{m}(v) / S_{b}(v)\right]\left[P_{m m}(v) / P_{s m}(v)\right]
$$

The first term is the ratio of the spectra from the microbolometer and bolometer. The second term accounts for the fact that the antenna couples to one spatial radiation mode and one polarization, whereas the bismuth film absorber of the composite bolometer couples to $A \Omega / \lambda^{2}$ spatial modes and two polarizations. $P_{m m}(v)$ and $P_{s m}(v)$ are just the standard expressions (Eqs. 2.13-2.14) for the multi-mode and single-mode power from a blackbody in a frequency bandwidth $d v$. It is easily shown that $P_{m m}(v) / P_{s m}(n) \propto v^{2}$.

As was discussed in Ref. 79, absorption losses in the dielectric lens and substrate can give rise to a spurious signal at the detector output. This effect can be particulariy troublesome at high frequencies where the number of spatial modes is very large, 


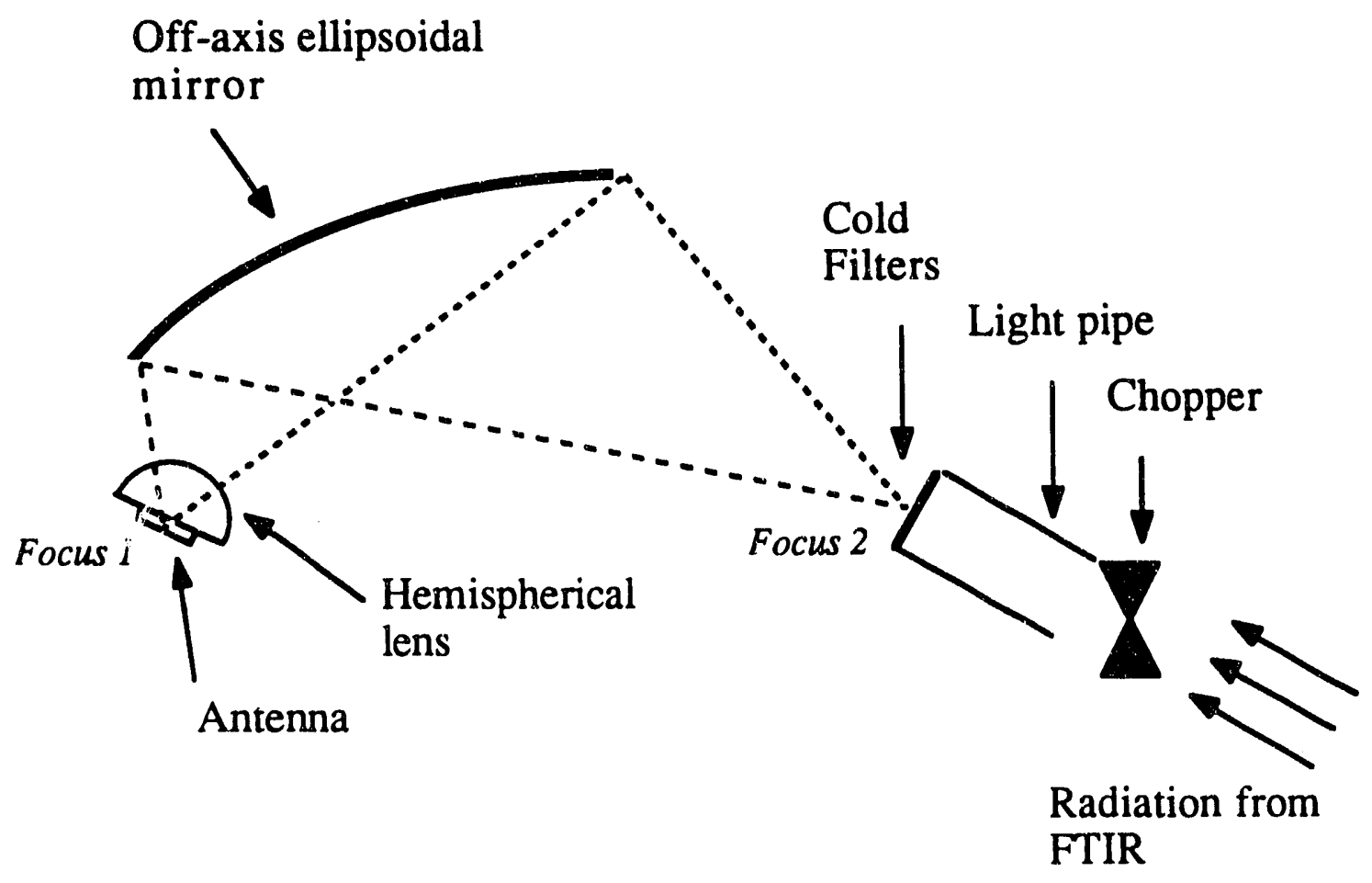

Fig. 5.7 Schematic of the experimental setup for measurement of the frequency dependent antenna response. Measurements of the optical efficiency were carried out by placing the blackbody reference at the second focal point of the mirror, in place of the light pipe. The distance between the focal points was $10 \mathrm{~cm}$. The angle subtended at the vertex of the antenna was $\approx 80^{\circ}$. 
and where dielectric losses become important. It is possible to avoid this effect by modulating the beam at a frequency which is higher than the inverse of the characteristic thermal response time of the dielectric. Fig. 5.8 shows the modulation frequency dependence of the microbolometer signal when illuminated with broadband radiation from the FTIR. The single frequency $90 \mathrm{GHz}$ measurements did not show this effect. For modulation frequencies above $\approx 300 \mathrm{~Hz}$, the signal is thus primarily due to the antenna response. This interpretation of the data was further verified by measuring the if frequency response of the microbolometer for various modulation frequencies. The microbolometer response for modulation frequencies of $150 \mathrm{~Hz}, 600 \mathrm{~Hz}$, and $800 \mathrm{~Hz}$, all as a function of if frequency is shown in Fig. 5.9. At low rf frequencies, where absorption losses in the dielectric are low and where the number of spatial modes is small, all spectra appear similar. At higher frequencies the absorption of radiation in the dielectric is observed for the spectrum obtained with a $150 \mathrm{~Hz}$ modulation frequency, but not for the $600 \mathrm{~Hz}$ or 800 $\mathrm{Hz}$ modulation frequency, which are both higher than the measured thermal rolloff frequency of the dielectric (Fig. 5.8). Subsequently, all spectra were obtained with a modulation frequency of $700 \mathrm{~Hz}$.

The un-normalized spectra measured with the microbolometer and with the constant throughput composite bolometer are shown in Fig. 5.10. Each FTIR spectrum is the convolution of the lamp spectrum, the beamsplitter efficiency, and of all other frequency dependent components in the instrument. The microbolometer spectrum decays much more rapidly than the bolometer spectrum. This behavior is not unexpected, since the antenna can couple to only one spatial radiation mode, whereas the bolometer, which measures the radiation from the constant throughput spectrometer, can couple to a number of modes which increase with frequency. For this reason, conventional bolometers are more useful for broadband spectroscopic applications in which many modes can be used. Another important feature in the microbolometer spectrum is the presence of the sharp 'wiggles'. Far infrared spectroscopists are very familiar with such features, they are generally 


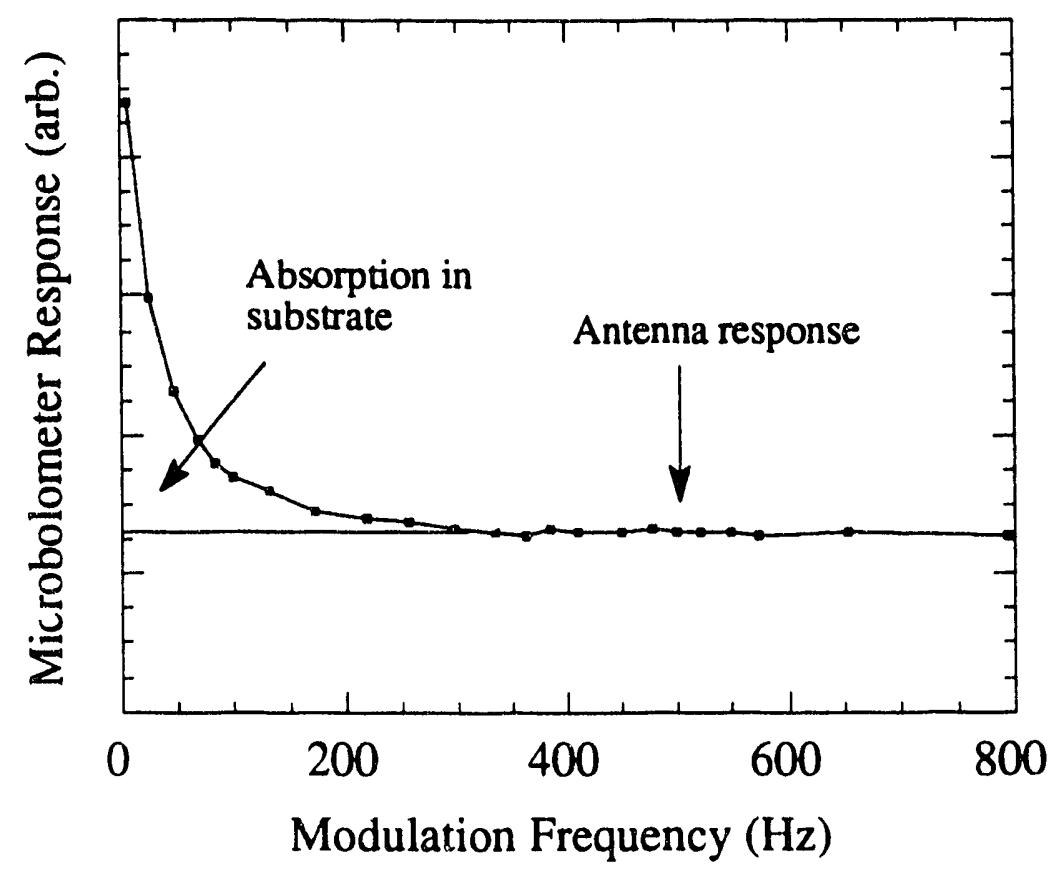

Fig. 5.8 Modulation frequency dependence of the microbolometer response, when illuminated with broadband radiation from the FTIR. For frequencies $<300 \mathrm{~Hz}$ the response is due to absorption of radiation in the dielectric lens and substrate. For frequencies above the inverse of this thermal response time, the response is due to radiation coupled to the antenna, and subsequently dissipated in the transition edge absorber/thermometer. For $90 \mathrm{GHz}$ radiation, incident from a waveguide coupled horn, the microbolometer response is independent of frequency up to $25 \mathrm{kHz}$ modulation frequency. 


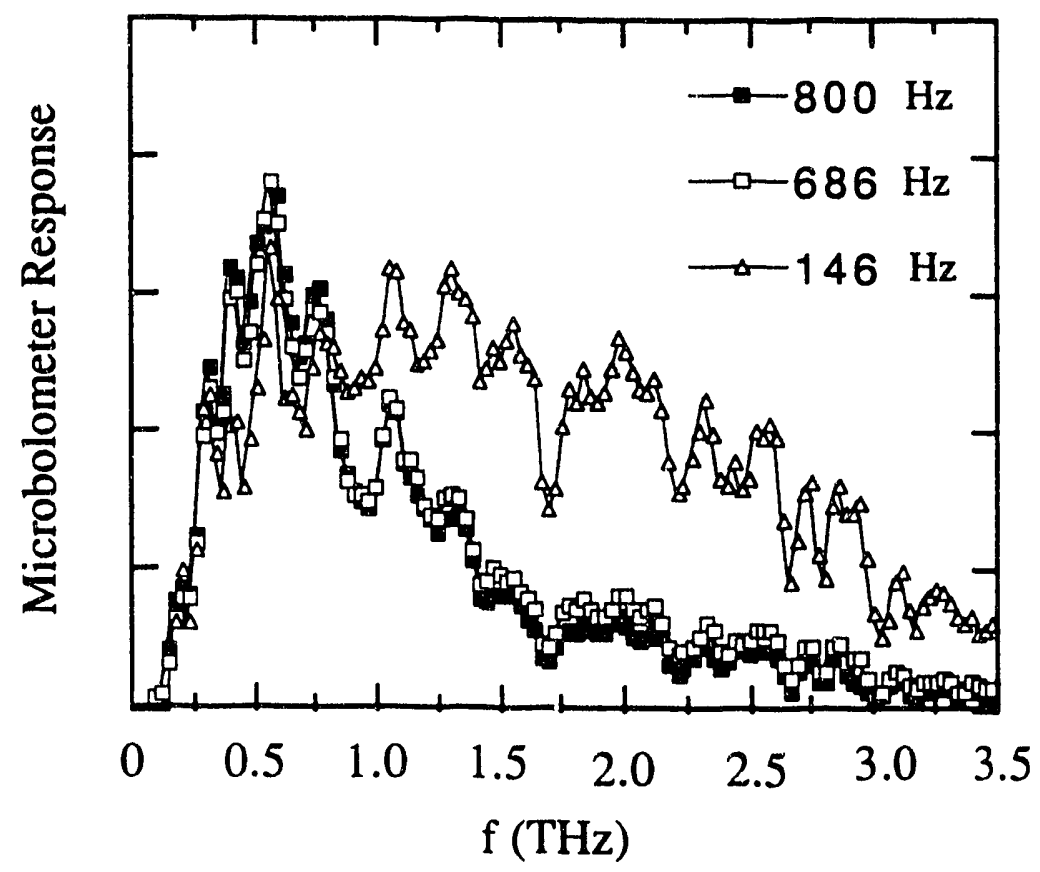

Fig. 5.9 Microbolometer rf response for various modulation frequencies. The response for modulation frequencies of $800 \mathrm{~Hz}$ is essentially identical to the response at $600 \mathrm{~Hz}$. For modulation frequencies below $\approx 150 \mathrm{~Hz}$, the response is very similar to the instrument spectrum obtained with a conventional (multi-mode coupled) composite bolometer. This indicates that, for low modulation frequencies, the microbolometer response is partially due to absorption of radiation in the bulk of the dielectric.

Subsequently, all spectra were obtained using a modulation frequency of $700 \mathrm{~Hz}$. 


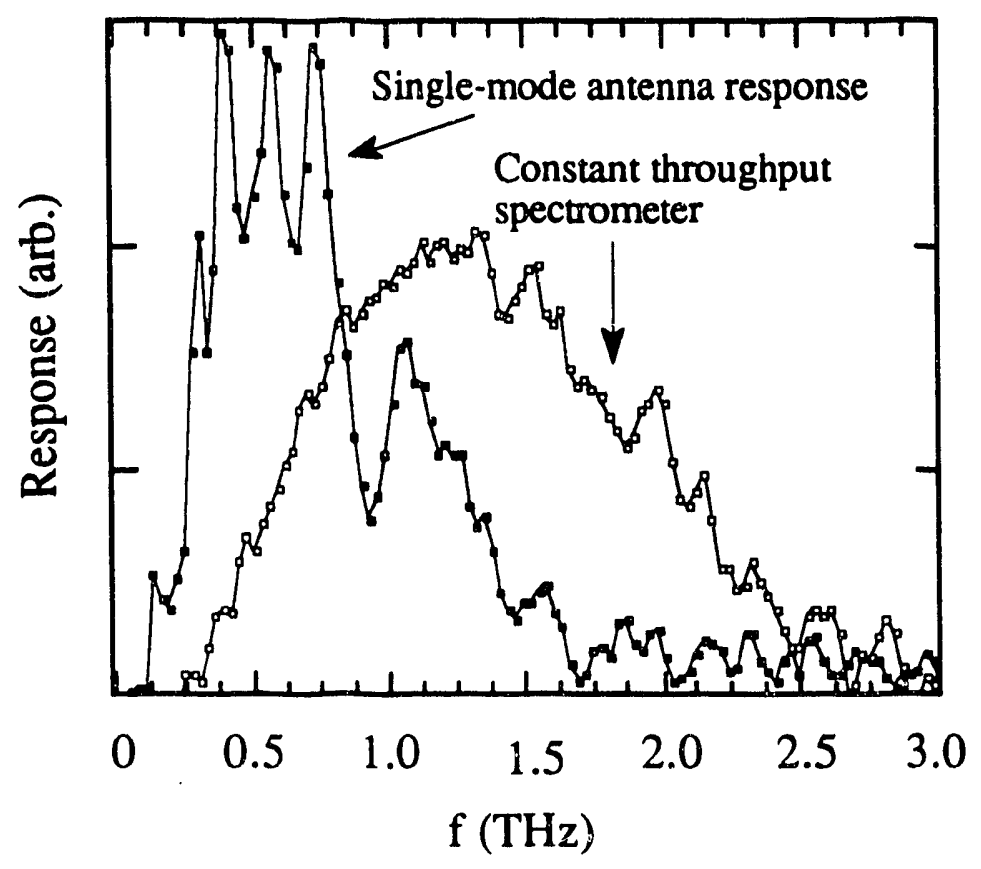

Fig. 5.10 Microbolometer power spectrum. Also shown is the FTIR spectrum obtained with a conventional composite bolometer. Both curves were obtained with a 0.003 " thick mylar beamsplitter. 
attributed to standing waves, commonly referred to as Fabrey-Perot fringes. For a dielectric slab of thickness $t$ and index of refraction $n$, the fringe spacing should be $\Delta(1 / \lambda)=1 /(2 n t){ }^{80}$ The wiggles in the antenna response however, are not equally spaced and thus cannot be explained in terms of standing waves in the optical path. This seems to suggest that the observed features are inherent to the antenna structure. In Fig. 5.11, the microbolometer spectrum has been divided by the bolometer spectrum. This ratio was further multiplied by the square of the frequency in order to account for the single-mode nature of the antenna. Figure 5.11 thus represents the frequency dependent optical efficiency of the log-periodic antenna. This is the first data of its kind, and is very important for the operation of all antenna coupled devices. There are four prominent features in the antenna response: a) The observed wiggles in the antenna response, b) the response of the antenna near the energy gap of the $\mathrm{Nb}$ antenna, c) the low frequency rolloff, and d) the high frequency rolloff. The following discussion will address each of these issues.

a) In the discussion of log-periodic antennas, I mentioned that for any log-periodic antenna that is not self complementary, the radiation pattern and the antenna impedance will repeat for frequencies $v_{0}$ and $\tau^{-1 / 2} v_{0}$, where $\tau$ is the periodicity factor of the antenna. In addition, for a self complementary structure, Booker's theorem states that the antenna impedance will be frequency independent for all frequencies. ${ }^{26}$ The question remains whether the radiation pattern will also become independent of frequency, or whether it will reflect the logarithmic periodicity of the antenna structure. The set of vertical arrows in Fig. 5.11. are spaced by the factor of $\tau^{-1 / 2}=1.41$, which is appropriate for the measured antenna. The agreement between the predicted and observed periodicity is almost perfect up to the 6th wiggle at $590 \mathrm{GHz}$. This result implies that the periodicity is caused by modulation of the radiation pattern, and hence of the power coupled to the antenna. It also implies that not all of the solid angle of the antenna is filled by radiation from the FTIR, since otherwise this effect would not be observed. 


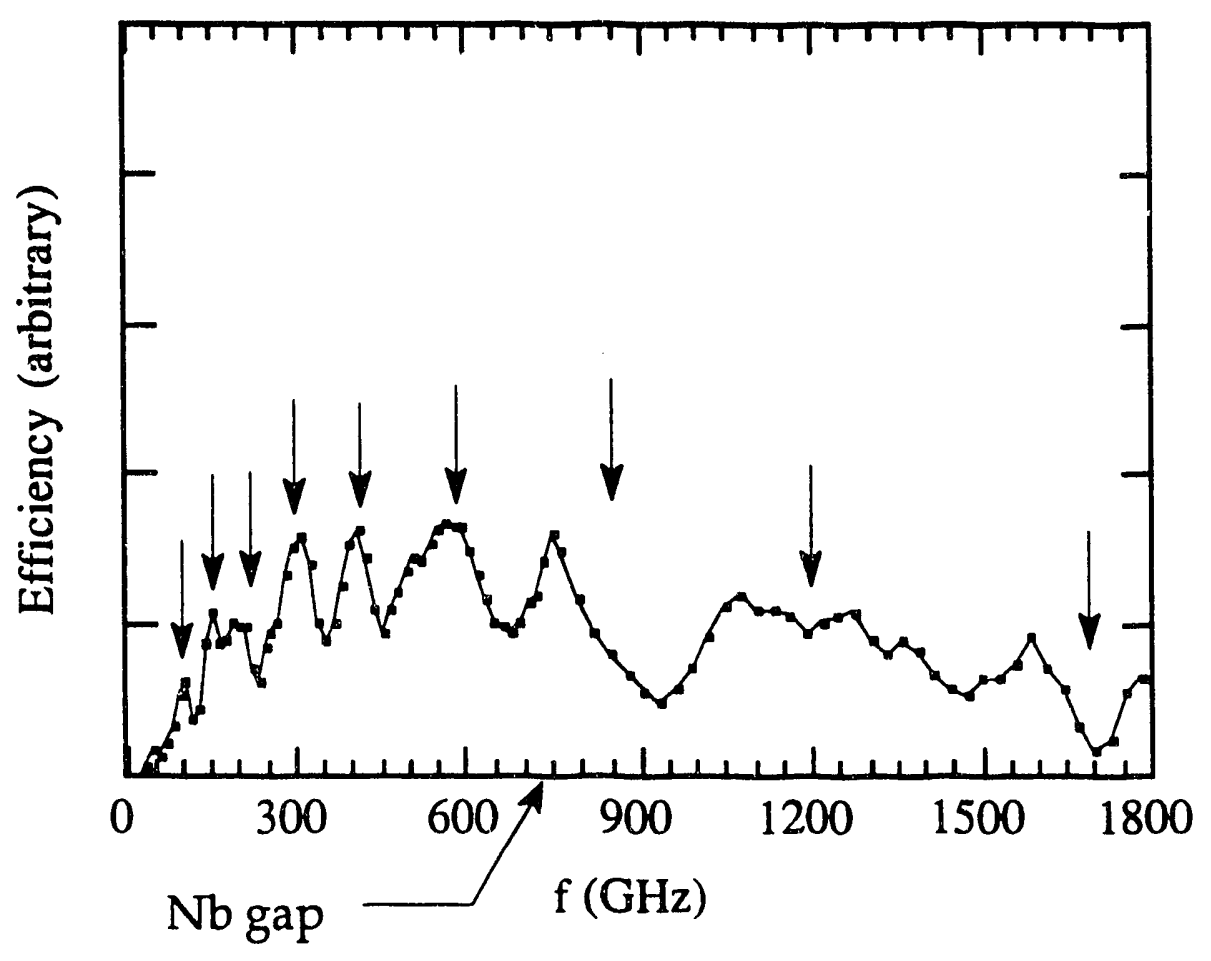

Fig. 5.11 Frequency dependent optical efficiency of the log-periodic antenna. The vertical arrows are spaced according the logarithmic periodicity of the antenna structure. The frequency corresponding to the energy gap of the $\mathrm{Nb}$ antenna is $740 \mathrm{GHz}$. 
b) The last three wiggles in the antenna response all appear at lower frequencies than what is predicted. One possible explanation for this effect is the onset of losses in the antenna at frequencies that are higher than the energy gap of $\mathrm{Nb}(740 \mathrm{GHz})$. The effect of such losses would be to decrease the apparent frequency of the wiggles at frequencies larger than $740 \mathrm{GHz}$. The observed rolloff at high frequencies however, may not be sufficient to cause such a shift in the peak position. An additional possibility may be due to the fact that the antenna is not strictly a self-complementary structure because its ends have been terminated. The short wavelength, high frequency currents may 'sense' this termination, thus causing a shift in the position of the peaks. The potential problem with this argument is that the antenna is terminated on the scale of $\approx 12 \mu \mathrm{m}$, whereas the peaks are shifted at frequencies corresponding to $\approx 100 \mu \mathrm{m}$. Additional experiments are required in order to fully understand this effect. These include measurements of the response of $\log$-spiral and bow-tie antennas that have a periodicity factor of $\tau=1$. Consequently, their radiation pattern will not depend on the rf frequency, ${ }^{76}$ and will thus improve our understanding of the antenna response above the gap.

c) The longest dimension of the antenna that maintains its complementary structure, corresponds to a frequency of $\approx 20 \mathrm{GHz}$. The measured low frequency cutoff on the other hand, occurs at a frequency of $\approx 100 \mathrm{GHz}$. As was previously discussed, the throughput of an antenna is approximately equal to the square of the wavelength. This implies that only part of the antenna area actually participates in the detection process. Thus, terminating the antenna some distance from its vertex should not influence the antenna properties for wavelengths that are shorter than this dimension. The exact frequency that corresponds to such a termination of the antenna is difficult to calculate and depends on the antenna structure. It is quite plausible though, that the log-periodic antenna would need to be made somewhat larger in order to efficiently couple this long wavelength radiation.

d) The response of the microbolometer decreases by $\approx 30 \%$ between $750 \mathrm{GHz}$ and $1.7 \mathrm{THz}$ (this is obtained by comparing the relative response at frequencies corresponding 
to the location of subsequent peaks). The main sources of loss at these frequencies are probably due to losses in the $\mathrm{Nb}$ antenna, and/or to dielectric losses in the crystal quartz lens $(0.25 "$ radius) and fused quartz substrate $(0.008 "$ thick). The measured transmittance of crystal quartz is $\approx 80 \%$ up to $1.2 \mathrm{THz}$, and then decreases rapidly to $\approx 5 \%$ by $3 \mathrm{THz}$. The measured transmittance of fused quartz is $\approx 80 \%$ up to $0.6 \mathrm{THz}$ and then decreases slowly to $5 \%$ by $3 \mathrm{THz}$.

In order to measure the coupling efficiency accurately, it is necessary to have a well calibrated source. In addition, the beam patterns of both the source and the detector must be matched to avoid coupling losses. The optical configuration shown in Fig. 5.7 is ideally suited for this measurement, if a blackbody source is placed at the second focus of the mirror. A variable temperature blackbody reference, similar to that described in Ref. 81, was constructed (Fig. 5.12). The absorber consisted of a circular cylinder of Ecosorb MF-1 $10,{ }^{40}$ with a $27.5^{\circ}$ conical cavity machined into the bottom surface. The absorber was maintained at a uniform temperature by gluing it to the inside of a copper cylinder with Stycast $2850 \mathrm{FT},{ }^{40}$ thus assuring adequate thermal contact. The temperature of the blackbody was varied by means of a thermometer and heater which were glued onto the copper cylinder, and by the weak thermal link between the absorber and the thermal bath. With this configuration, it was possible to vary the temperature of the blackbody from $2.5 \mathrm{~K}$ to $100 \mathrm{~K}$. The beam divergence of the blackbody was defined by a Winston light concentrator, ${ }^{82}$ and was chosen so as to completely fill the ellipsoidal mirror which in turn subtended an angle of $\approx 85^{\circ}$ with respect to the vertex of the antenna. The benefit of this configuration is that a large fraction of the antenna beam can be filled by the blackbody radiation. The optical bandwidth was determined by a cold flourogold low pass filter which had an effective bandwidth of $\approx 720 \mathrm{GHz}$. A tuning fork resonant chopper, ${ }^{67}$ operating at a frequency of $210 \mathrm{~Hz}$, was used :o modulate the incident power. The spurious signall arisitig from the absorption of radiation in the quartz lens and the substrate should be negligibly srnall fo: this particular measurement because of the extremely low 


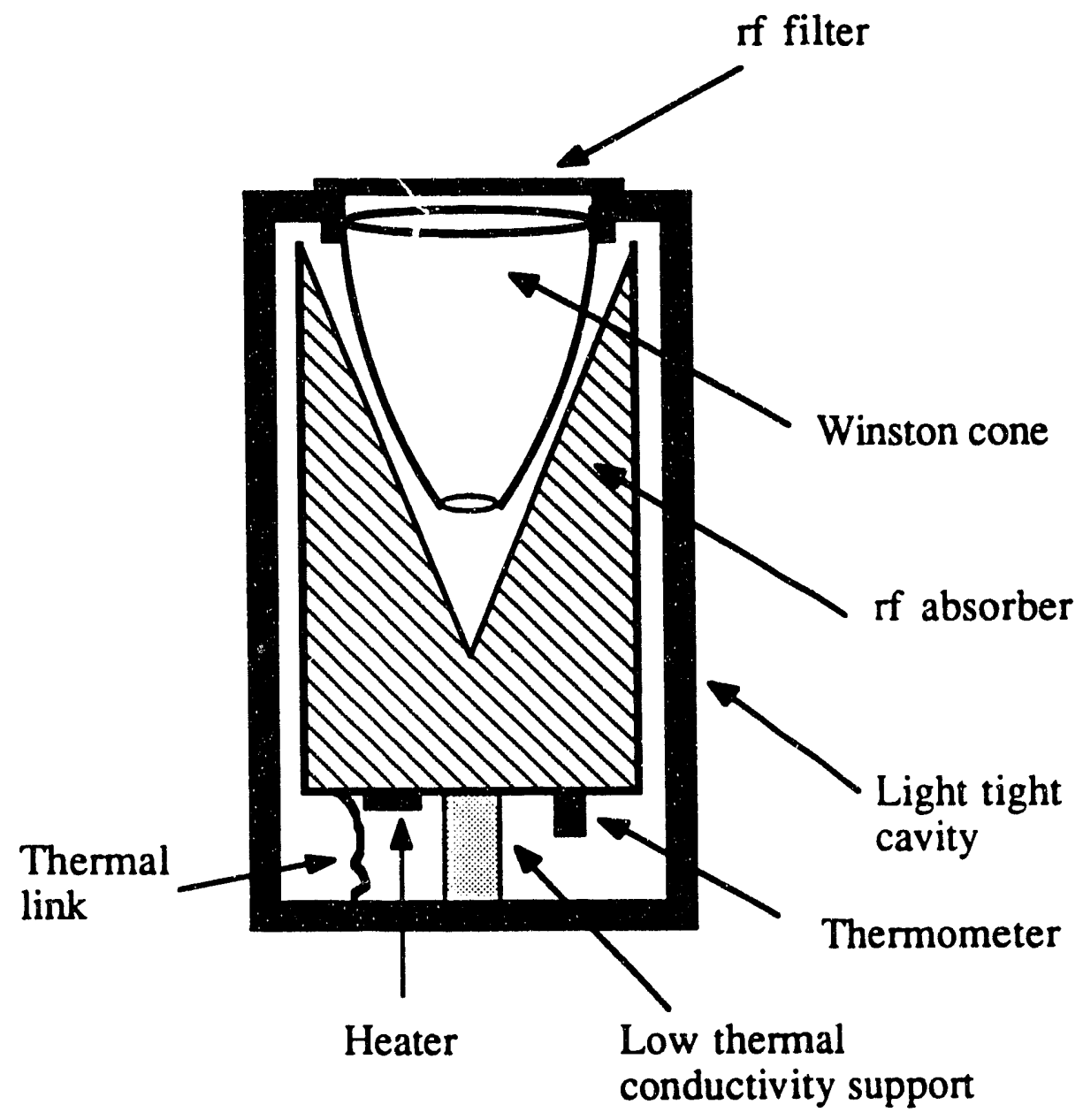

Fig. 5.12 Schematic of the variable temperature blackbody reference. With this conñiguration it is possibie to vary the temperature of the biackbocily betweenn $2 \mathrm{~K}$ to $100 \mathrm{~K}$. 
losses in quartz for this low frequency range. This was verified by observing that the dependence of the microbolometer response on the temperature of the blackbody was identical to the temperature dependence of the single mode power from a blackbody (Fig. 5.13). If radiation were dissipated in the quartz, rather than being coupled to the antenna, then the temperature dependence would have been that of the power from a multimode blackbody source. Our measurements indicated an optical efficiency of $1.5 \%$ for the quasi-optical system. The following corrections were applied in order to extract the antenna efficiency: a) a factor of 1.25 for the reflection of radiation at the quartz lens/vacuum interface, b) a factor of 10.1 due to the mismatch between the impedance $R=4 \Omega$ of the thermally active region, and the impedance $Z_{\text {ant. }}=120 \Omega$ of the antenna. With these corrections we estimate the antenna efficiency at $\approx 20 \%$.

\subsection{Design of tuning Elements and Narrow Bandpass Filters}

When typical superconductors with large value of normal state conductivity $\sigma_{n}$ are used, the if resistance of the thermometer will be much lower than the $\approx 120 \Omega$ real terminal impedance of typical self-complementary antennas or the even higher impedances available for matching to waveguides. This mismatch can be minimized by the use of low conductivity metals and/or by the use of long thin films. For long films however, the geometrical inductance of an isolated thermometer strip can become significant at $\approx 2 \mathrm{THz}$. This further complicates the matching problem. Some of these difficulties can be avoided by integrating the thermometer in a superconducting microstrip line. Once this is done, impedance transformers can be used to match the film resistance to the antenna, and filters can be introduced to control the bolometer if bandpass.

Because the thermometer may not be small enough that it will appear as a lumped element at rf-frequencies, it is treated as a lossy microstrip. The impedance of the

thermometer strip $Z_{\text {bolo }}$ of length $l$ and width $w$, can be calculated from ${ }^{29,64}$ 


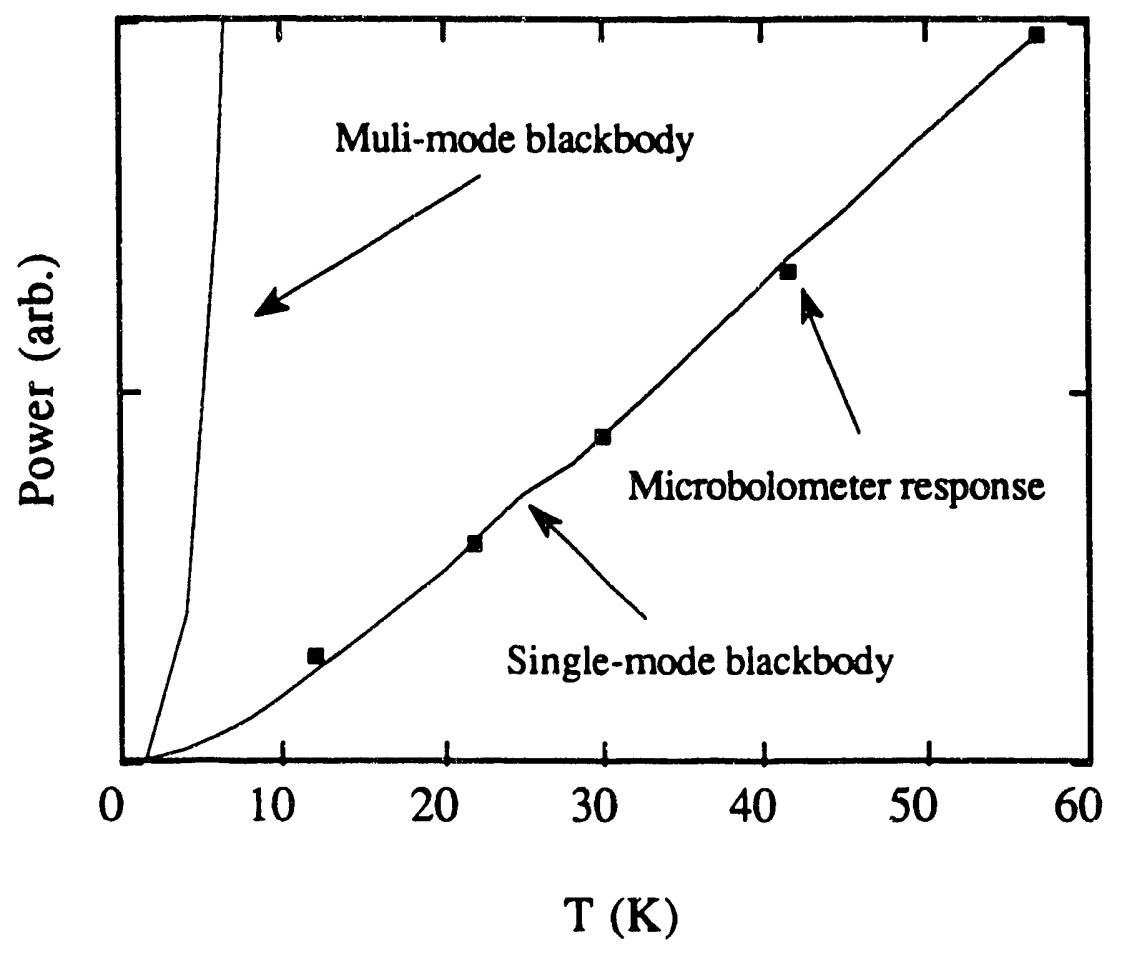

Fig. 5.13 Microbolometer response to the variable temperature blackbody source (squares). Also shown is the temperature dependence of the power from single-mode and a multi-mode blackbody. The excellent agreement between the data and the single-mode result, indicates that absorption of radiation in the dielectric lens is a negligible effect for this particular experiment. 


$$
\mathrm{Z}_{\mathrm{bolo}}=(\mathrm{Z} / \mathrm{Y})^{1 / 2_{\tanh \gamma l}}
$$

where

$$
\mathrm{Z}=\left(\mathrm{i} \mu_{0} 2 \pi v d+\mathrm{Z}_{\mathrm{S}_{\mathrm{a}}}+\mathrm{Z}_{\mathrm{S}_{\mathrm{b}}}\right) / \mathrm{k} w
$$

is the series impedance per unit length of the transmission line,

$$
\mathrm{Y}=\mathrm{i} \varepsilon_{0} \varepsilon 2 \pi v \mathrm{k} w / \mathrm{d}
$$

is the shunt admittance per unit length of the line, and

$$
\gamma=(Z Y)^{1 / 2}
$$

is the propagation constant. Here $d$ is the dielectric thickness, $Z_{S_{a}}$ and $Z_{S_{b}}$ are the surface impedances of the lower groundplane and upper thermometer strip respectively, $\mathrm{k}$ is the fringing factor of the stripline, ${ }^{83}$ and $\varepsilon$ is the dielectric constant. When $v$ is small compared to the energy-gap frequencies of the groundplane $(\approx 40 \mathrm{GHz}$ for $\mathrm{Nb}$ at $\mathrm{T}=0 \mathrm{~K}), \mathrm{Z}_{\mathrm{S}_{\mathrm{a}}}$ is well approximated by the predictions of the two fluid model of Eq. 3.5. As previously discussed, the surface impedance of the thermometer strip $\mathrm{Z}_{\mathrm{S}_{\mathrm{b}}}$ can be described by the normal state limit.

An additional advantage of integrating the thermometer in a superconducting microstrip line is that it becomes possible to include efficient matching networks, made from transmission line elements in the bolometer design. When microstrip lines produced by conventional lithographic techniques are used for the network elements, the optimum range for the thermometer resistance is between $2 \Omega$ and $30 \Omega$. Combining microstrips with coplanar waveguides would extend the useful resistance range up to more than $100 \Omega$, and 
therefore enlarge the range of applications. The benefits of such networks are not restricted to badly matched thermometers. A major advantage of this configuration is that lithographed filter elements can be incorporated with relative ease. These lithographed filters can have superior performance over conventional filters which tend to exhibit high frequency leakage at submillimeter wavelengths.

A specific example of a poorly matched thermometer is a strip made from a film of bcc-Ta with $\mathrm{T}_{\mathrm{c}}=4.2 \mathrm{~K}, l=10 \mu \mathrm{m}, w=2 \mu \mathrm{m}$, and $\mathrm{t}=1000 \AA$ (these dimensions are similar to the devices that were fabricated and measured in the previous sections). The thermometer is incorporated in the microstrip configuration with a $d=6000 \AA$ thick $\mathrm{SiO}_{2}$ dielectric and a thick $\mathrm{Nb}$ ground plane. For this specific example the real part of $\mathrm{Z}_{\text {bolo }}$ is $2.7 \Omega$ above $T_{c}$, which presents a very poor match to the antenna. The imaginary part of $\mathrm{Z}_{\text {bolo }}$ is $4.7 \Omega$ above $\mathrm{T}_{\mathrm{c}}$ and $6.6 \Omega$ in the kinetic inductance region at $300 \mathrm{GHz}$. For calculations it is reasonable to use $\mathrm{Z}_{\mathrm{bolo}}=(2.7+\mathrm{i} 5.4) \Omega$ at $300 \mathrm{GHz}$. Using conventional microwave theories, ${ }^{84} \mathrm{~J}$. Mees has designed quarter-wave transformers with several elements. Fig. 5.14 shows the calculated coupling coefficient $C_{\text {rf }}$ versus frequency for a) the above thermometer on a ground plane but without tuning elements, b) with one transforming element and c) one transforming element plus an additional stub that cancels the reactance of the thermometer. As a further demonstration of the power of these techniques we have designed 7-element Chebyshev bandpass filters with $30 \%$ and $10 \%$ bandwidths. These are shown in d) and e) respectively. ${ }^{84,85}$ A typical filter layout is shown at the right. These calculations were done with lossless microstrip elements coupled to the lossy thermometer. Little change is seen in the filter characteristics when microstrip losses as large as $600 \mathrm{~dB} / \mathrm{m}$ are included in the calculations. Losses below $10 \mathrm{~dB} / \mathrm{m}$ have been measured in $\mathrm{Nb}$ trilayer technology at $100 \mathrm{GHz}{ }^{86}$ 


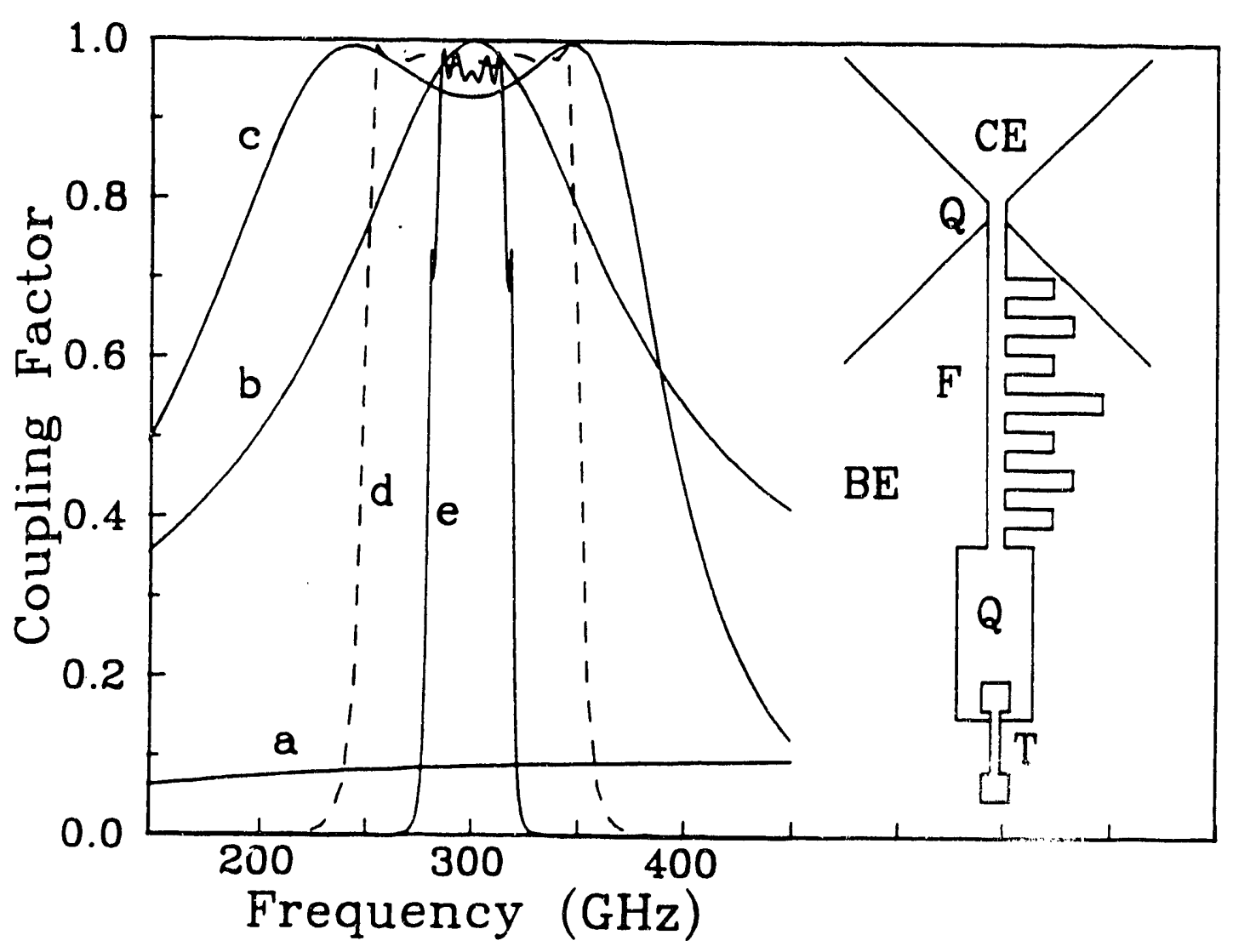

Fig. 5.14 The frequency dependent RF-coupling factor for several examples: a) For the thermometer on a ground plane but without any matching network. b) The same thermometer with one quarter wave transforming element. c) Same as b) plus an open-ended stub which cancels the reactance of the bolometer. d) Thermometer with a 7-element Chebyshev filter and a $30 \%$ bandwidth. e) Same as d) but with a $10 \%$ bandwidth. A typical layout of a Chebyshev filter with transforming sections is shown at right. The antenna terminals are labeled base electrode $B E$, and counter electrode $\mathrm{CE}$. The other elements are two impedance transformers $\mathrm{Q}$, the filter $F$ and the thermometer $T$. The dielectric layer which covers the base electrode is not shown. 


\subsection{Conclusions}

A prototype low- $T_{c}$ antenna-coupled microbolometer has been successfully fabricated and tested. The real test for any prototype device however, is whether it can successfully compete with the existing technology. For the low- $\mathrm{T}_{c}$ microbolometer, the question is whether it can provide a higher sensitivity than the best conventional composite bolometers operating at the same temperatures, and within the same frequency band. Our estimates indicated that the sensitivity of the microbolometer could indeed exceed that of the best available detectors of submillimeter and millimeter waves by as much as an order of magnitude. Our measurements indicated that the thermal and electrical properties of the microbolometer are in excellent agreement with the predicted behavior. In order to compete successfully with the existing technology however, several obstacles must be overcome. The impedance of the absorber/thermometer strips must be a close match to the antenna impedance of $\approx 120 \Omega$. This means that either the number of squares in the strips should be increased, or different transition-edge thermometers should be investigated. Furthermore, it is necessary to solve the materials problems for a microbolometer operating at or below $300 \mathrm{mK}$, since it is for these operating temperatures where the low- $T_{c}$ microbolometer could potentially replace the existing technology. It appears that either $\mathrm{Ti}$ or the $\beta$-phase of Ta could be useful, since their measured electrical resistivities are both in the right range to solve the impedance matching problem. Furthermore, it is crucial to understand the properties of the antenna structures. The low- $T_{c}$ transition-edge absorber/thermometer is the ideal sensor for these measurements, and can, in principle, be used to measure the response of any antenna. In section 5.5, I described the first direct measurement of the frequency dependent optical efficiency of a log-periodic antenna using the low- $\mathrm{T}_{\mathrm{c}}$ microbolometer. It is clear that a systematic study of the properties of these structures is necessary before the low- $T_{c}$ microbolometer made into a useful device. The potential merits of this device, however, clearly favor further development of these concepts. 


\section{Chapter 6:-Design_Analysis of a Hot-Electron_Microbolometer}

\subsection{Introduction}

The idea of an antenna-coupled hot-electron bolometer which uses a superconductinginsulator-normal (SIN) junction to as a sensitive thermometer was developed while I was thinking about ways to reduce the thermal conductance from the thermally active region to the environment, and hence to increase the sensitivity of the detector. Our preliminary estimates indicate that it may be possible to obtain an order of magnitude higher sensitivity over the superconducting microbolometer, and up to two orders of magnitude improvement over conventional composite bolometers in the far IR. Unlike the transition edge microbolometer, the requirements for temperature regulation are less stringent. A schematic of the proposed configuration is shown in Fig. 6.1. A thin strip of metal with micron dimensions serves as a resistive load to thermalize the infrared curreits from the antenna. The temperature rise of the electrons in the metal absorber is measured from the temperature dependence of the current-voltage (I-V) characteristics of a superconductorinsulator-normal metal (SIN) junction, where part of the metal strip forms the normal electrode. Thermal isolation is provided by the weak electron-phonon interactions at low temperatures and by the reflection of electrons at the interface between the metal absorber and the superconducting antenna. Efficient radiation coupling can be achieved by means of a planar lithographed antenna. Although this concept has not been tested, I have decided to include the design analysis in this dissertation.

An important application for the SIN readout scheme is in fundamental measurements of electron heating effects in thin metal films. Although the basic mechanism for these effeits is well understood, there are yet a few unresolved issues. These include the effects

of phonon dimensionality in the metal film on the thermal isolation of the electrons, ${ }^{87}$ as well as the effects of impurity scattering. Using the temperature readout scheme proposed here, it should be possible to make direct measurements of the electron temperature in 


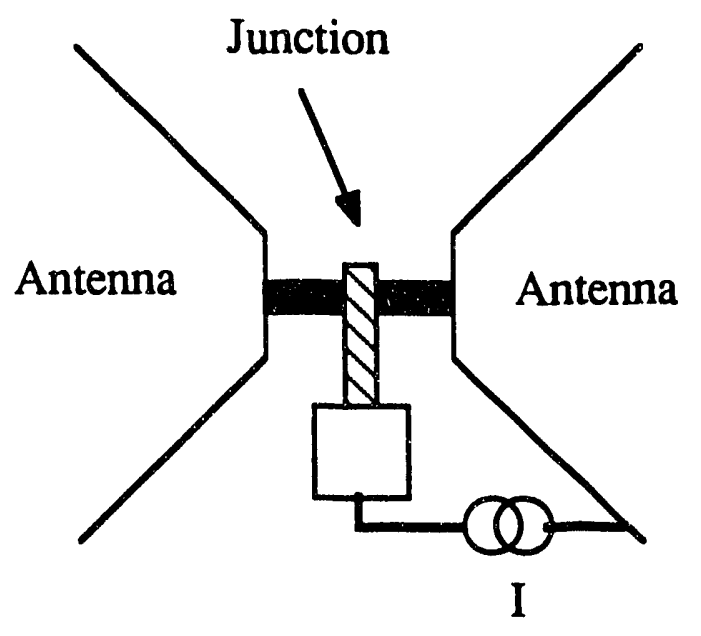

Fig. 6.1 Schematic of the radiation coupling and temperature readout configuration. The rf current from the superconducting antenna is dissipated in the resistive metal strip (black strip), the resulting temperature rise of the electrons in the strip are measured as a change in the voltage across the junction which is biased at a constant current I. The contact to the superconducting electrode is made of a superconductor whose $T_{c}$ is much higher than that of the electrode. 
the film, with a sensitivity that is several orders of magnitude higher than previous investigations using SQUDD noise thermometry techniques. ${ }^{61,88}$

In this chapter I will briefly review the basic theory hot-electrons in metals, previous measurements of this effect, and methods to maximize the thermal isolation of the electrons. I will then discuss the temperature readout scheme and its noise contribution to the NEP of the hot-electron microbolometer. The design calculations will are presented in section 6.4. In section 6.5, estimates of the sensitivity and usefulness of a SIN tunnel junction for measuring fundamental electron heating effects will be presented.

\subsection{Energy Dissipation}

In the proposed geometry, the absorbed power can be dissipated via three paths. The first is direct heat flow from the electrons in the strip to the substrate via electron-phonon coupling and phonon transport. At temperatures above $\approx 10 \mathrm{~K}$, the electrons and phonons in the strip are in thermal equilibrium, and the thermal conductance is dominated by the spreading resistance arising from the thermal conductivity of the substrate, as is the case for the high- $T_{c}$ microbolometer described in chapter 3. At lower temperatures, the thermal boundary resistance can contribute significantly to the thermal isolation. This property has been used to construct the sensitive low- $T_{c}$ microbolometers which were described in chapter 5. At very low temperatures and for small film volumes however, an additional effect has to be considered. For temperatures less than $\approx 1 \mathrm{~K}$, the inelastic collision processes (energy loss processes) for electrons in a metal are very infrequent. As a result, the electrons become thermally decoupled from the lattice and heat up as they absorb power. The theory of hot-electrons is relatively well understood and in reasonable agreement with experiment. ${ }^{88,89}$ The simplest treatment assumes that the electrons and phonons are each in internal thermal equilibrium and are in weak contact with one another. The deformation potential approximation and Fermi's Golden rule are then used to calculate the electron-phonon relaxation time, and hence the temperature rise of the electrons 
relative to the phonons for a given dissipated power $\mathrm{P}$. The standard analysis gives

$$
P=\Sigma V\left(T_{e}^{5}-T_{p}^{5}\right)
$$

where $\mathrm{V}$ is the volume of the metal, $T_{e}$ is the electron temperature, $T_{p}$ is the phonon temperature. Here $\Sigma$ is a constant of order $10^{2} \mathrm{WK}^{-5} \mathrm{~cm}^{-3}$ which depends on the density of states at the Fermi surface, the Fermi velocity, the speed of sound, and the mass density per unit volume of the metal. The thermal conductance $G_{e-p}$ between the electrons and the phonons (for temperature differences such that $T_{e} \approx T_{p}$ ) is then given by

$$
\mathrm{G}_{\mathrm{e}-\mathrm{p}}=5 \Sigma \mathrm{VT} \mathrm{T}^{4}
$$

In contrast to the thermal boundary conductance $G_{b d}$ which is proportional to the contact area of the film with the substrate, $G_{e-p}$ is proportional to the volume of the film, the reason being that both the number of phonons and the number of electrons are proportional to the film volume. The temperature dependence $\mathrm{c}_{\mathrm{f}} \mathrm{G}_{\mathrm{e}-\mathrm{p}}$ is proportional to the fourth power of the temperature, whereas $G_{b d}$ is proportional to the third power of the temperature. Thus, $\mathrm{G}_{\mathrm{e}-\mathrm{p}}$ will limit the thermal transport for thin films at low temperatures, typically for a film thickness $\leq 1000 \AA$ at $\mathrm{T} \leq 1 \mathrm{~K}$.

Typical experiments on hot-electrons usually measure the temperature difference between the electrons and the phonons and thus the thermal conductance $G\left(=G_{e-p}\right)$ between the two systems. The inelastic relaxation time is then obtained from $\tau_{e-p}=C / G$, where $\mathrm{C}$ is the heat capacity of the electron system. Copper is one of the more widely studied metals and has a measured electron-phonon conductance of $\mathrm{G} \approx 2 \times 10^{3} \mathrm{VT}^{4} \mathrm{~W} / \mathrm{K}$, where $V$ is the volume in $\mathrm{cm}^{3}$ and $T$ is the temperature in Kelvin. ${ }^{61,89,90}$ The calculated inelastic scattering rate $\tau_{\mathrm{e}-\mathrm{p}} \approx 10.6 / \mathrm{T}^{3}$ ns. The measurements of Wellstood et al. on CuAu thin films give $G \approx 1.2 \times 10^{4} \mathrm{VT}^{4} \mathrm{~W} / \mathrm{K} .{ }^{91}$ For a typical active bolometer volume of $1 \mu \mathrm{m}^{3}$ at 
an operating temperature of $\mathrm{T}=100 \mathrm{mK}$ the thermal conductance can be as low as $\mathrm{G} \approx 10^{-13} \mathrm{~W} / \mathrm{K}$. This is several orders of magnitude smaller than typical values for conventional bolometers operated at the same temperature, and can be very useful for low background, narrow bandwidth applications, where the temperature rise of the electrons will be small.

In order to utilize fully this effect it is necessary to insure that the contribution of all other energy relaxation processes to the thermal conductance are also small. The contact area between the metal absorber and the antenna terminals provides an alternate path for heat dissipation. In addition to the phonon relaxation mechanism discussed above, there exists the possibility that electrons could also transfer energy across this interface. However, if the antenna is made from a superconductor whose $T_{c}$ is higher than the operating temperature of the device, then the Andreev reflection of electrons at the interface between the metal absorber and the superconducting antenna traps the hot-electrons and

hence the absorbed energy in the active region. ${ }^{15}$ The same principle was used to isolate the transition edge thermometer from the antenna for the low- $T_{c}$ microbolometer.

An additional mechanism for energy dissipation is due to the presence of the SIN junction. Electrons which tunnel through the insulating barrier remove energy from the active region and thus provide an additional mechanism for heat dissipation. If the contact to the superconducting electrode is made with another superconductor whose energy gap much is larger than that of the electrode then the Andreev reflection of quasiparticles at this interface will also trap the energy in the active region. The contribution of the superconducting electrode to the volume of the active region would have a negligible effect on the sensitivity if it can be made small in comparison with the volume of the metal strip.

\subsection{Temperature Readout}

As previously discussed, the temperature rise of the electrons is detected by a SIN junction, where part of the metal absorber forms the normal electrode. The usefulness of 
the junction as a thermometer for the electron temperature stems from the exponential dependence of the tunneling current on the temperature. It is possible to show that to first order, the quasiparticle current through a SIN junction, whose electrodes are at different temperatures, depends only on the temperature $\mathrm{T}$ of the electrons in the normal electrode and is identical to the case where both electrodes have the same temperatures. ${ }^{92}$ If the junction is biased such that $\mathrm{eV} \ll \Delta$ and $\mathrm{kT} \ll<\Delta$ then the quasiparticle current is given by

$$
\mathrm{I}=(\mathrm{kT} / \mathrm{eR}) \sinh (\mathrm{eV} / \mathrm{kT}) \text {, }
$$

where $R=R_{N}(k T / 2 \pi \Delta)^{1 / 2} \exp (\Delta / k T)$ is the dynamic resistance of the junction at low bias, $R_{N}$ is the normal resistance of the junction, and $\Delta$ is the energy gap of the superconducting electrode. ${ }^{92}$ If the junction is biased with a constant current then the voltage responsivity is

$$
S_{o}=(1 / G) d V / d T=-(1 / G)(k / e)[(\Delta / k T+1 / 2) \tanh (e V / k T)-(e V / k T)]
$$

For a given choice of $\Delta$ and $\mathrm{kT}$ it can be shown that the responsivity is maximized when $\cosh ^{2}(\mathrm{eV} / \mathrm{kT})=(\Delta / \mathrm{kT}+1 / 2)$.

In order to estimate the contribution of the temperature readout to the NEP it is necessary to understand the noise mechanisms in the junction. The voltage fluctuations of a biased SIN junction can be derived using standard many-body techniques and in general, are related to the quasiparticle current-voltage characteristics and the frequency. For most applications, the frequencies of interest (the modulation frequency) are such that $h \omega_{0}<<\mathrm{eV}$. In this low frequency limit the voltage noise across the junction is independent of frequency and is given by $\mathrm{V}_{\mathrm{n}}{ }^{2}=(\mathrm{dV} / \mathrm{dI})^{2} 2 \mathrm{e} I \operatorname{coth}(\mathrm{eV} / 2 \mathrm{kT}),{ }^{93}$ or

$$
\mathrm{V}_{\mathrm{n}}{ }^{2}=2 \mathrm{kTRtanh}(\mathrm{eV} / \mathrm{kT}) \operatorname{coth}(\mathrm{eV} / 2 \mathrm{kT}) / \cosh (\mathrm{eV} / \mathrm{kT})
$$


When $\mathrm{eV}<<\mathrm{kT}$, Eq. 6.5 reduces to Johnson noise arising from the dynamic resistance of the junction, when $\mathrm{eV} \gg \mathrm{kT}$ the noise is due to the shot noise arising from the discrete nature of the charge carriers. The contribution of the temperature readout to the NEP is given by the ratio of the voltage noise and the responsivity (NEP) ${ }_{S I N}=V_{n} / S_{0}$. In general,

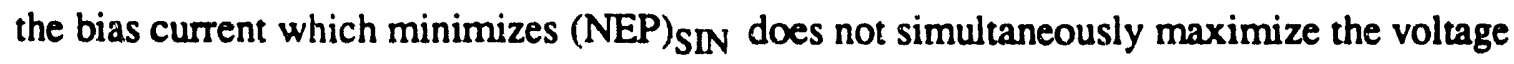
responsivity. The voltage responsivity $S_{0}$ and its contribution to the noise equivalent power $\mathrm{V}_{\mathrm{n}} / \mathrm{S}_{\mathrm{o}}$, are both shown as a function of the bias across the junction in Fig. 6.2. For bias values such that $2 \leq \mathrm{eV} / \mathrm{kT} \leq 6$, the noise changes by less than a factor of two.

The low thermal conductance that couples the electrons to their environment places an additional restriction on the bias current. The IV power which is dissipated in the vicinity of the junction could significantly raise the temperature of the electrons, and consequently reduce the sensitivity of the device. An optimum value for this temperature rise can be calculated if the background infrared power dissipated in the detector is known. For purposes of this discussion we require that bias power not raise the temperature of the electrons by a fraction $\zeta$ of the operating temperature, where $\zeta=0.3$. If we assume that all of the power is dissipated normal electrode, then this requirement reduces to IV $<G \zeta T$. In reality, some of the power will be transferred to the phonons and will subsequently be removed through the substrate.

\subsection{Design Calculations}

Due to the large number of parameters, the optimization of the hot-electron microbolometer is somewhat complicated. In general, it is advantageous to pick the smallest possible volume for the metal absorber so as to reduce the thermal conductance. Once the operating temperature has been determined, it then becomes possible to estimate the therm conductance between the electrons and the phonons. One then proceeds by finding the bias current which minimizes the contribution of the temperature readout to the NEP. It is then necessary to ensure that the bias heating will not raise the temperature of 


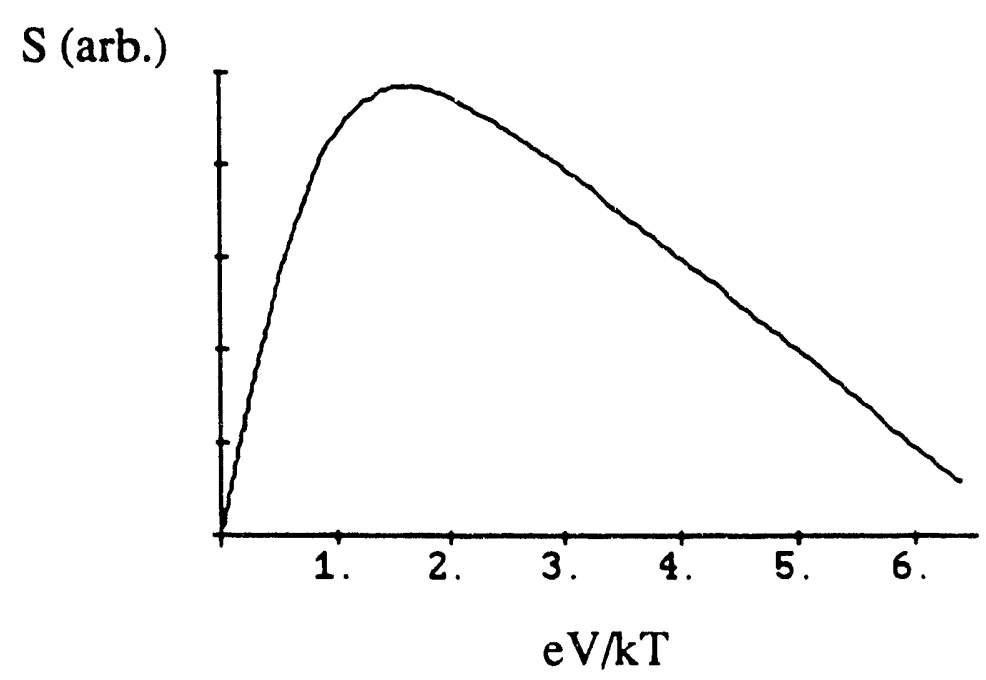

a)

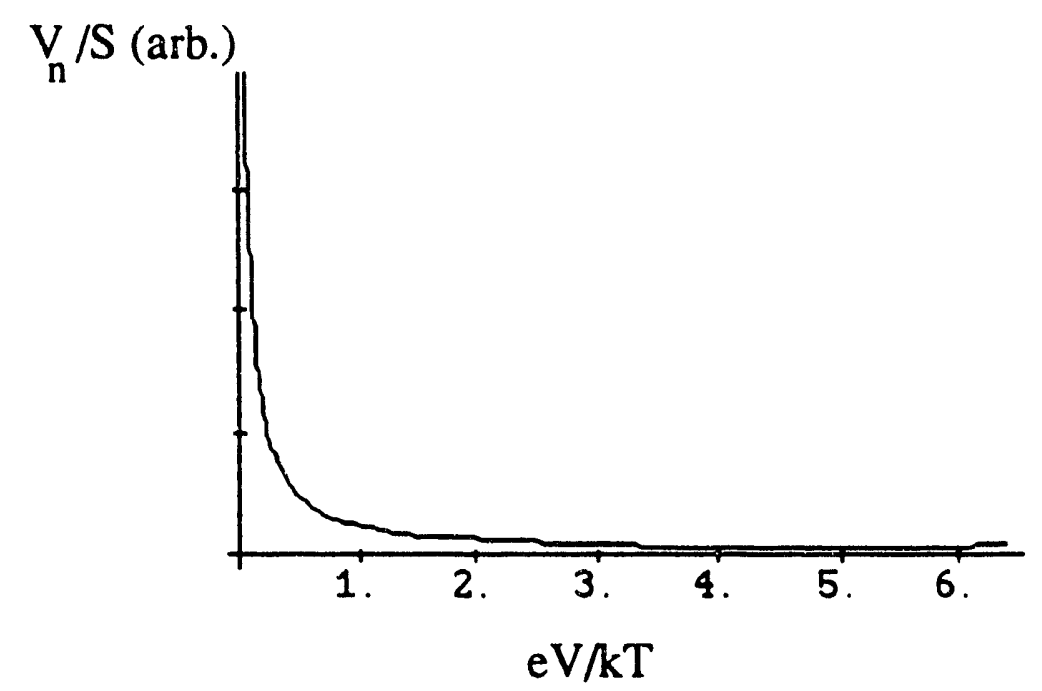

b)

Fig. 6.2 a) Bias dependence of the voltage responsivity. b) Bias dependence to the NEP of the voltage noise from the junction. For both cases it was assumed that $\Delta / \mathrm{k} T=6.5$, and that $R_{i v}=100 \Omega$. 
the electrons by a significant factor. An important feature of the hot-electron microbolometer is that the time constant $\tau$, which equals the electron-phonon relaxation time $\tau_{e-p}$, is much smaller than is required for most applications. Consequently, the tradeoff between speed of response and sensitivity which is inherent to the optimization of conventional composite bolometers, is avoided.

As a specific example of a device whose performance would be very beneficial to the astrophysics community, we assume an active volume of $2 \times 6 \times 0.05 \mu^{3}$ at operating temperatures of $100 \mathrm{mK}$ and $300 \mathrm{mK}$. The ratio of the energy gap of the superconducting electrode to the operating temperature is assumed to be $\Delta / \mathrm{kT} \approx 6.5$, and the normal state resistance of the junction is assumed to be $R_{N}=100 \Omega$. For an operating temperature of $\mathrm{T}=100 \mathrm{mK}$ the superconducting electrode could be made of $\mathrm{Ti}$, where $\mathrm{s}$ at $\mathrm{T}=300 \mathrm{mK}$ a useful choice would be aluminum. Although other metals may be more appropriate, we use the value of $\mathrm{G}$ which was measured for $\mathrm{Cu}$ films ${ }^{61}$ for the purpose of the present analysis. The figures of merit of the hot-electron microbolometer as well as the bias current, the output voltage, and the total voltage noise $\mathrm{V}_{\mathrm{n}}$ out resulting from the contributions of energy fluctuations in the active region and the fluctuations in the junction are listed in Table 6.1. For an operating temperature of $100 \mathrm{mK}$ the sensitivity of this device is almost two orders of magnitude higher than the best ayailable conventional composite bolometer. The total voltage noise at the output of the detector is $\mathrm{V}_{\mathrm{n}}{ }^{\text {out }} \approx 1 \mathrm{nVHz}{ }^{-1 / 2}$, which is comparable with the input voltage noise of $\approx 1-2 \mathrm{nVHz}^{-1 / 2}$ of commercially available low noise JFET amplifiers at frequencies above any $1 / \mathrm{f}$ noise knee.

The $\mathrm{rf}$ impedance of the metal strip $\mathrm{Z}_{\text {bolo. }}$ is given by $\mathrm{Eq} .3 .6$, where the surface impedance $Z_{S}$, is just the normal state limit of Eq. 3.5. The bolometer impedance then is the sum of the dc resistance of the strip and its geometrical reactance. The strip can also be integrated in the stripline geometry that was discussed in section 5.6. 


\begin{tabular}{|l|l|l|}
\hline$T(m K)$ & 100 & 300 \\
\hline$G(W / K)$ & $1.2 \times 10^{-13}$ & $9.7 \times 10^{-12}$ \\
\hline$\tau_{\mathrm{e}-\mathrm{p}}(\mu \mathrm{s})$ & 10.6 & 0.4 \\
\hline $\mathrm{S}_{\mathrm{O}}(\mathrm{V} / \mathrm{W})$ & $3.4 \times 10^{9}$ & $4.2 \times 10^{7}$ \\
\hline $\mathrm{I}(\mathrm{nA})$ & 1.9 & 5.7 \\
\hline $\mathrm{V}(\mu \mathrm{V})$ & 14.0 & 42.1 \\
\hline$(\mathrm{NEP}) \mathrm{SIN}\left(\mathrm{WHz}^{-1 / 2}\right)$ & $3.7 \times 10^{-20}$ & $5.2 \times 10^{-18}$ \\
\hline$\left(4 \mathrm{kT}{ }^{2} \mathrm{G}\right)^{1 / 2}\left(\mathrm{WHz}^{-1 / 2}\right)$ & $2.6 \times 10^{-19}$ & $7.0 \times 10^{-18}$ \\
\hline$(\mathrm{NEP})\left(\mathrm{WHz}^{-1 / 2}\right)$ & $2.6 \times 10^{-19}$ & $1.0 \times 10^{-17}$ \\
\hline $\mathrm{V}_{\mathrm{n}}{ }^{\text {uut }}\left(\mathrm{nVHz}{ }^{-1 / 2}\right)$ & $2.6 \times 10^{-9}$ & $1.0 \times 10^{-9}$ \\
\hline
\end{tabular}

Table 6.1. Parameters for a device operated at $100 \mathrm{mK}$ and $300 \mathrm{mK}$. The normal electrode is assumed to be $500 \AA$ thick and have an active area of $2 \times 6 \mu \mathrm{m}^{2}$. The junction is assumed to have a normal state resistance $R_{N}=100 \Omega$. 


\subsection{Tunneling as a Probe of Hot-Electrons}

The subject of hot-electrons at low temperatures is of interest not only from a theoretical point of view, but also because the optimization of many types of devices requires an understanding of this effect.' In particular, the effects of hot-electrons can be detrimental to the performance of low temperature SQUIDs ${ }^{91}$ and single electron tunneling devices. $^{94}$ The few direct measurements of electron heating phenomena have commonly employed dc SQUIDs to measure the Johnson noise arising from the resistance of a thin metal strip and the temperature of the electrons. The tlectrons were heated above the phonon temperature by passing an electric current through the resistive strip, and the resultant rise in the Johnson noise was measured. An alternative measurement method is proposed here which uses the temperature dependence of the current-voltage characteristic of a superconductor-insulator-normal metal (SIN) junction to directly measure the temperature of the electrons in the normal metal electrode. The temperature of these electrons can be raised above the lattice by passing a current through the normal electrode. The advantages of this method are the relative simplicity of the measurements and the very high temperature resolution compared with the SQUID method. A schematic of the proposed configuration is shown in Fig. 6.3. This configuration is identical to the one proposed in the previous sections, except that here a known amount of power is dissipated in the metal strip by passing a known dc current through that strip. The temperature rise of the electrons is then monitored as a change in the voltage across the SIN tunnel junction, where part (or all) of the normal metal forms the normal electrode.

As discussed in section 6.3, for a SIN junction biased at a constant current, and for $\mathrm{kT}<<\Delta$, the change in the voltage across the junction, due to a change in the electron temperature is given by

$$
S=d V / d T=(k / e)[(\Delta / k T+1 / 2) \tanh (e V / k T)-(e V / k T)]
$$




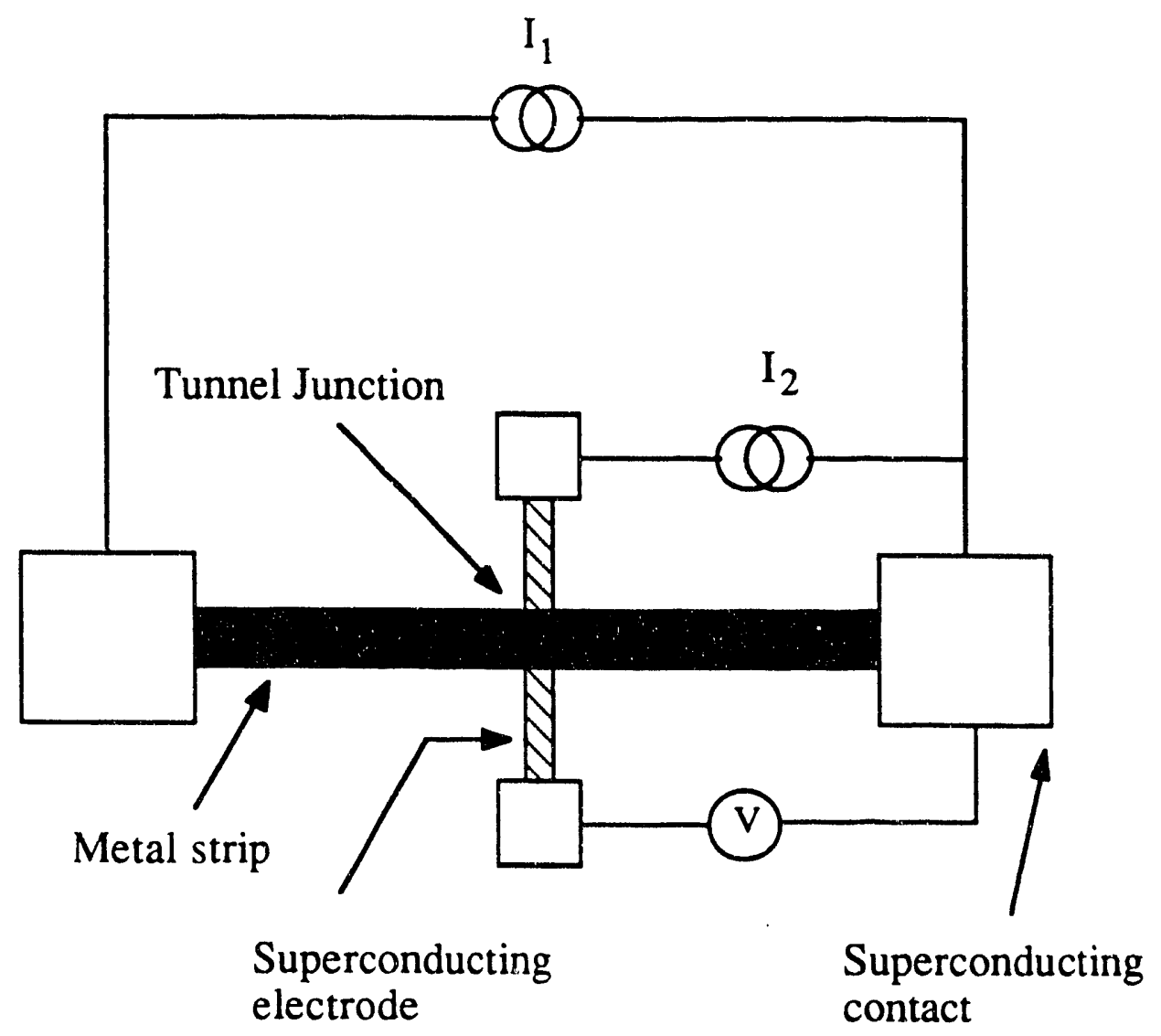

Fig. 6.3 Schematic of the experimental configuration for the proposed measurement of hot-electron effects in thin films. The junction is biased with a current $\mathbf{I}_{\mathbf{2}}$ and the voltage $V$ across the junction is monitored. A current $I_{1}$ is passed through the metal strip, and subsequently dissipates electrical power in that strip. The resultant temperature rise of the electrons in the normal electrode is measured as a change in the voltage $\mathrm{V}$. The superconducting contacts to both the metal strip and to the superconducting electrode (which has a lower energy gap than that of the contact) ensures that the dissipated energy cannot escape via electrons from the active region. 
The voltage $V_{n}$ noise across the junction (Eq. 6.5) determines the ultimate limitation of the temperature sensitivity. The minimum detectable temperature change in a $1 \mathrm{~Hz}$ noise bandwidth is then given by the ratio $V_{n} / S$, whose minimum value determines the optimum bias current. The optimization requirements for this device are similar to those of the hotelectron microbolometer, and thus will not be repeated.

As a specific example illustrating the potential of this technique I consider an operating temperature of $100 \mathrm{mK}$, a normal state resistance of $R_{N}=500 \Omega$, and $\Delta / \mathrm{k} T \approx 6$. Under these conditions the optimum operating point for measurement of the electron temperature occurs at a bias current of $1 \mathrm{nA}$ with a resultant sensitivity of $5=10^{-4} \mathrm{~V} / \mathrm{K}$, and minimum detectable temperature change of $30 \mathrm{nK}$ in a $1 \mathrm{~Hz}$ noise bandwidth. This is equivalent to a temperature resolution of $\Delta \mathrm{T} / \mathrm{T}=1.5 \times 10^{-7}$ in a one second integration time. For comparison, the temperature resolution of SQUID techniques is only about $10^{-2}$.

\subsection{Conclusions}

The estimated sensitivity of the hot-electron microbolometer can exceed that of the best available conventional bolometers by two orders of magnitude, with a time constant which is three orders of magnitude smaller. Also, it is possible to construct multiplexed systems by designing on-chip networks with different bandpass filters fed from a single antenna. Using well developed reproducible lithographic techniques it should be possible to make powerful array receivers with multiple spectral bands. It thus appears that the hot-electron microbolometer could be the detector of choice in applications requiring very high sensitivities and array compatibility. Furthermore, it should be possible to make sensitive measurements of electron heating phenomena with a temperature resolution of 1 part in $10^{7}$. 


\section{Chapter 7: Proposed Measurements of Quasiparticle Trapping_Effects}

\subsection{Introduction}

For the device architectures that are described in this dissertation, it is necessary to make electrical contact to a very small region, while simultaneously restricting the flow of energy from that region. For other devices, such as $\mathrm{x}$-ray detectors, it is necessary to funnel energy into a small active region..$^{95-97}$ Both of these device concepts exploit the effects of Andreev reflection, commonly referred to as quasiparticle trapping, to influence the flow of energy. In 1964, A. F. Andreev considered the question of what happens to an electron in a normal metal as it propagates towards an interface between the metal and a superconductor. ${ }^{15}$ His calculation indicated that if an electron has an energy that is smaller than the energy gap of the superconductor, it can propagate into the superconductor only if it pairs with another electron. To conserve energy and momentum, a reflected hole with a reversed group velocity and a negative effective mass is formed, and travels back along the original electron path with the same energy as the incident electron. This process causes a thermal resistance at the interface.

The effects of Andreev reflection have been previously measured in a variety of experiments. The increase in the thermal resistance of superconductors in their intermediate state, ${ }^{15}$ and the electrical resistance at normal/superconducting interfaces, ${ }^{98-101}$ have been successfully explained by assuming that the energy carriers are reflected at the interface between the normal and superconducting phases. The subharmonic energy gap structure and excess current observed in the I-V curves of superconducting weak links and normalsuperconducting microconstriction contacts, was also explained in terms of the Andreev reflection. ${ }^{102-105}$ More recently, quasiparticle trapping has become a very important issue for the development of x-ray detectors. A variety of experiments have been carried out in order to investigate the possibility of directing non-equilibrium quasiparticles that are produced in the superconductor, towards a small area on the superconductor that 
contains a tunnel junction. ${ }^{95-97}$

Approximately three months prior to the writing of this dissertation, we invented an experiment that could directly probe the transmission coefficient $T(E)$ across a single metal/superconducting interface, as a function of the energy gap of the superconductor. Although the measurements have yet to be completed (due primarily to materials related difficulties), I have decided to present a short summary of this work since it is intimately related to several of the device concepts presented in this dissertation, and will be completed in the near future. This will be an important experiment since many of the early measurements were made on superconductors in the intermediate state, which consisted of many normal/superconducting interfaces, rather than on a single interface. In addition, these experiments probed the temperature, rather than the energy gap dependence of this effect, thus complicating the interpretation of the results, since it is necessary to understand the temperature dependence of the phonon conduction process. More recent measurements, which use non-equilibrium quasiparticles that are generated in the superconductor to measure the collection efficiency of quasiparticle traps, do not provide useful information on the transmission coefficient.

In this chapter, I will present the basic theory of Andreev reflection, and derive the temperature dependence of the thermal conductance at the interface between a metal and a superconductor. In section 7.3, I will discuss the measurement scheme, and will then present some very preliminary results.

\subsection{Theoretical Background}

For purposes of this discussion, it is convenient to present Andreev's analysis in qualitative terms, ${ }^{98}$ rather than describing it in detail. Fig. 7.1a shows the spatial variation of the energy gap $\Delta$ across the interface between a metal and a superconductor. The energy gap varies within a few coherence lengths $\xi$, from zero in the metal to $\Delta_{\infty}$ far within the superconducting region. The excitation spectra in the normal and superconducting metals 
are shown in Fig 7.1b. Consider an electron $A$ whose energy $E$ (measured relative to the Fermi energy) is smaller than $\Delta_{\infty}$. The electron will be reflected at the point where its energy is equal to $\Delta$, and the whole process of reflection can be seen as a transition from a state A through $A^{\prime}$ to $A^{\prime \prime}$, which is the same as $B^{\prime \prime}$, and back through $B^{\prime}$, to the final holelike state B. Charge conservation is achieved by the injection of two electrons into the superconducting ground state. An electron whose energy is greater than $\Delta_{\infty}$, will be partially reflected as a hole. A normal electron with the same energy as the incident electron, will then appear in the superconductor with a probability $T(E)$. The process of Andreev reflection thus gives rise to a thermal resistance at the interface since the energy carriers are efficiently blaked from entering the superconductor.

Andreev calculated $T(E)$ for a step-like potential where $\Delta=0$ in the normal metal and $\Delta=\Delta_{\infty}$ in the supercond ictor. $^{15} \mathrm{He}$ used Gor'kov's equations of motion for quasiparticles in the superconductor, ${ }^{106}$ and matched the appropriate boundary conditions at the interface, to give

$$
T(E)=\left[\begin{array}{cc}
2 \frac{\left(E^{2}-\Delta_{\infty}^{2}\right)^{1 / 2}}{E+\left(E^{2}-\Delta_{\infty}^{2}\right)^{1 / 2}} & E>\Delta_{\infty} \\
0 & E<\Delta_{\infty}
\end{array}\right] .
$$

The exact form of $T(E)$ for a realistic interface is difficult to calculate. Hsiang 101 considered the effect of different spatial distributions of $\Delta$ on $T(E)$, and concluded that it is reasonable to use Andreev's approximation for transport calculations.

It is now possible to calculate therrmal resistance for electrons crossing an interface between a metal and a superconductor. The calculation is analogous to the that for the thermal boundary resistance for phonons crossing an interface between two dielectrics (chapter 3), except that here the transmission coefficient depends on the carrier energy. The power flow across the interface, for electrons incident on the superconducting side, 

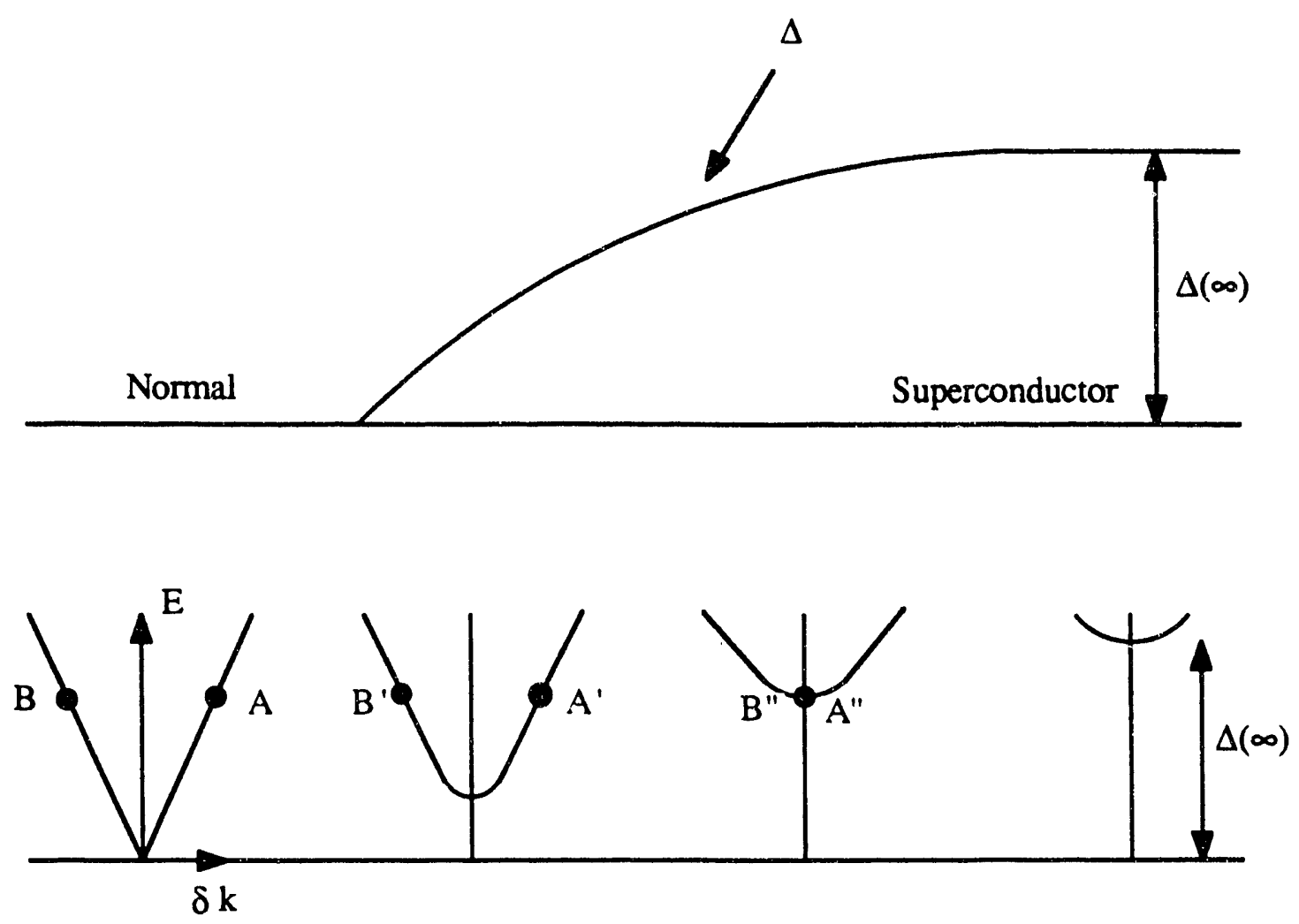

Fig. 7.1 a) Assumed variation of the energy gap $\Delta$ with position across a superconducting-normal interface. b) The variation of the excitation spectrum for different positions close to the interface. Also illustrated is the reflection of an excitation of energy that is incident from the normal side in the state A, and reflected into the state B. The vertical axis is the excitation energy, the horizontal axis is the momentum measured radially from a point on the Fermi surface. 
is the integral over all energies and incident angles of the number of electrons that are incident on a unit area $\mathrm{A}$ per unit time, times the electron energy $\mathrm{E}$, times the transmission probability $T(E)$. This can be written as

$$
P(T)=\frac{A}{4 \pi} \iiint_{\Phi \Theta E} N(E, T) E v(E) \cos \Theta T(E) \sin \Theta d \Phi d \Theta d E .
$$

Here $N(E, T)=D(E) f(E, T)$ is the product of the electron density of states $D(E)$ per unit volume times ihe Fermi-Dirac occupation fictor $f(E, T), v(E)$ is the electron propagation velocity, and $T(E)$ is the transmission prciaty for an electron to be transmitted across the interface. The expression $d \Omega=\sin \Theta d \Phi d \Theta$ is the solid angle, where $\Theta$ is the angle between the electron propagation direction and the normal to the interface, and $\Phi$ is the azimuthal angle.

For temperatures such that $k T \ll<\Delta$ and for a step-like interface, Andreev derived an analytical solution to Eq. 7.2. ${ }^{15}$ For this particular case, the density of states and the electron velocity can both be approximated by their respective values at the Fermi surface. The transmission probability, for $E \approx \Delta$, is approximately $T(E) \approx 2^{3 / 2}(E / \Delta-1)^{1 / 2}$. The power flow directed from the normal to the superconducting phase is then given by,

$$
P(T)=A(2 k T / \pi \Delta)^{3 / 2}\left(E_{F} \Delta\right)^{2} \hbar^{-3} v_{F}^{-2} \exp (-\Delta / k T)
$$

Here $E_{F}$ is the Fermi energy and $v_{F}$ the Fermi velocity. If the temperatures of the normal metal and superconducting sides are equal, the flux $\mathrm{P}$ is compensated by an identical flux flowing in the opposite direction. If a temperature difference $\delta \mathrm{T}$ exists across the interface, then the resulting flux is $(\partial \mathrm{P} / \partial \mathrm{T}) \delta \mathrm{T}$, and the thermal conductance is

$$
\mathrm{G}=\mathrm{A}(2 / \pi)^{3 / 2} \mathrm{E}_{\mathrm{F}}{ }^{2} \mathrm{k} \hbar^{-3} \mathrm{v}_{\mathrm{F}}{ }^{-2}(\Delta / \mathrm{kT})^{1 / 2} \exp (-\Delta / \mathrm{kT})
$$


For a typical metal, $E_{F} \approx 10 \mathrm{eV}$ and $v_{F} \approx 10^{8} \mathrm{~cm} / \mathrm{s}$. Using these approximations, the thermal conductance is $G \approx A 10^{-3}(\Delta / k T)^{1 / 2} \exp (-\Delta / k T) \mathrm{V} / \mathrm{K}$, where $A$ is in $\mu \mathrm{m}^{2}$. When $\Delta / \mathrm{kT} \gg 1$, the electronic contribution to the thermal conductance of the interface is negligible, while for $\Delta / \mathrm{kT} \leq 1$ the thermal conductance can become significant compared with the phonon contribution to the thermal conductance.

\subsection{Measurement Scheme}

The goal of the proposed experiment is to measure the electronic contribution to the thermal conductance across a metal/superconducting interface, as a function of the energy gap of the superconductor. The transmission coefficient can then be inferred from Eq. 7.2. This is a not an easy experiment because energy transport due to phonons crossing the interface can complicate the analysis. Furthermore, measurements of thermal conductances in bulk samples inevitably require corrections to account for temperature gradients through the bulk material. These difficulties can be overcome by reducing the size of the experiment to micron dimensions. A schematic of the proposed experiment is shown in Fig. 7.2. A transition edge thermometer operating at temperature $\mathrm{T}$ is in electrical contact with two superconducting pads whose transition temperature is much higher than $T$. The pads are in contact with thick metal pads that are located close to the active region. The purpose of the metal pads is to function as heat sinks. The device is placed in a parallel magnetic field $\mathrm{H}$. The experiment consists of measuring the I-V characteristics of the transition edge thermometer as a function of the applied magnetic field. The I-V curve contains information on the thermal conductance between the thermometer strip and its environment (see chapter 2), while the magnetic field can be used to vary the energy gap of the superconducting contacts. In what follows, I will briefly review the magnetic field dependence of the superconducting energy gap, followed by a detailed discussion of the energy flow processes from the thermometer strip to its environment.

It is well known that for a bulk superconductor, a sufficiently large magnetic fieid 


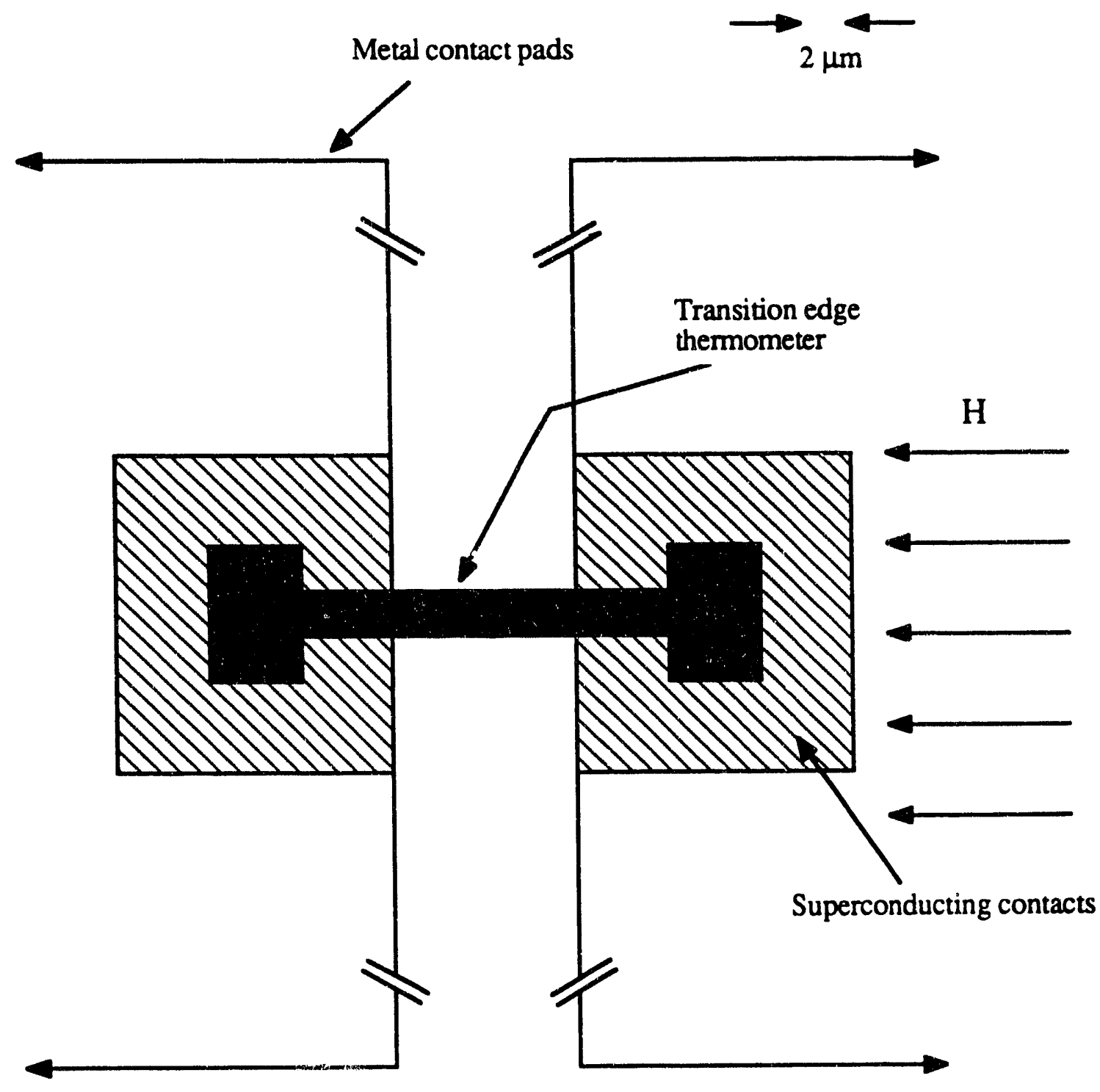

Fig. 7.2 Schematic diagram of the experimental configuration. 
applied parallel to the surface, will cause the superconductor to revert to its normal state with $a$ corresponding absorption of a latent heat, indicative of a first order phase transition. $10 \%$ inis means that the superconducting energy gap will drop discontinuously to zero at the critical field. For thin films (thickness $\leq 1000 \AA$ ) on the other hand, the energy gap decreases smoothly to zero as the magnetic field is increased up to the critical field, indicative of a second order phase transition. ${ }^{108}$ The difference between these two cases is that for a bulk sample the energy associated with the exclusion of the magnetic field gives rise to the latent heat at the critical field, whereas for a thin sample, the field completely penetrates the superconductor, and hence there is no latent heat when the film undergoes a phase transition. In $1961, \mathrm{D} . \mathrm{H}$. Douglass, Jr. ${ }^{108}$ realized that the effect of the magnetic field on the energy gap could be described by the Ginzburg-Landau phenomenological theory, ${ }^{109}$ provided that the energy gap was identified with the order parameter, a result which had been obtained by Gor'kov from the microscopic theory. ${ }^{106}$ This theory predicts a continuous decrease of $\Delta$ to zero as $H->H_{c}$ for films whose thickness $d<5^{1 / 2} \lambda$, where $\lambda$ is the temperature dependent London penetration depth, and a discontinuous drop in $\Delta$ when $d<5^{1 / 2} \lambda$. For $d / \lambda \ll<$, the theory predicts $\Delta(H) / \Delta(0)=\left[1-\left(H / H_{c}\right)^{2}\right]^{1 / 2}$, where $H_{c}$ is the critical field of the film. For intermediate values of $d / \lambda$, the magnetic field dependence of $\Delta$ is solved numerically. Tunneling measurements by Douglass, ${ }^{108}$ and by Douglass and Meservey, ${ }^{110}$ as well as interpretations of thermal conductivity measurements of Morris and Tinkham, ${ }^{111}$ all tend to confirm the prediction of the theory in the region of its validity, near $T_{c}$ and in the local limit $\xi>\lambda$, where $\xi$ is the effective coherence length. For our proposed experiment the Ginzburg-Landau theory is no longer strictly valid, because the temperature is much smaller than $T_{c}$. The deviations from this theory however are not significant. For $\mathrm{T} / \mathrm{T}_{\mathrm{c}}=0.36$, the measured gap is $\approx 20 \%$ smaller than the theoretical prediction. ${ }^{112}$ Tinkham derived a phenomlogical theory that extends the Ginzburg-Landau theory to lower temperatures. ${ }^{112}$ In this his theory, the dependence of the energy gap on the magnetic field at low temperatures and for $d / \lambda \ll<$ is $\Delta(H) / \Delta(0)=1-\left(H / H_{c}\right)^{2}$. 
This result fits the experimental data to within $5 \%$.

As previously discussed, the goal of this experiment is to measure the electronic contribution to the thermal conductance across a metal/superconducting interface. Measurements of the I-V characteristics of the thermometer however, yield information on the total thermal conductance from the strip to its environment. It is thus necessary to understand the various energy relaxation processes and to isolate the electronic contribution. The thermal circuit for this experiment is shown in Fig. 7.3. Power is dissipated in the electrons, which are assumed to be in internal thermal equilibrium, and subsequently leaves the active region via the following possible paths:

a) Electrons cross interface and relax to the heat sink. The energy flow for this path is determined by the thermal conductance $\mathrm{G}$ of the interface (the quantity of interest), and the thermal conductance $G_{1}$ to the heat sink.

b) Electrons relax to the phonons (which are also assumed to be in internal thermal equilibrium). The phonons can then either cross the interface with the underlying substrate, or cross the interface with the superconducting contacts. Both of these relaxation processes are controlled by the thermal boundary resistance, and are well described by the acoustic mismatch model (see chapter 4 ). It should be noted that although the electrons have relaxed to the lattice, they can still transfer energy across the interface.

In order to simplify the analysis, I will assume that the electrons are in thermal equilibrium with the phonons. This implies that $G_{e-p}$ is much larger than $G, G_{1}$, and $G_{b d}$. The measured thermal conductance is then due to both phonon and electron transport, and is given by

$$
G_{m}=\left(G^{-1}+G_{1}^{-1}\right)^{-1}+G_{b d}
$$

Here I have combined the phonon transport to the underlying substrate and across the interface with the superconductor into one term, $G_{b d}$. In order to extract the thermal 


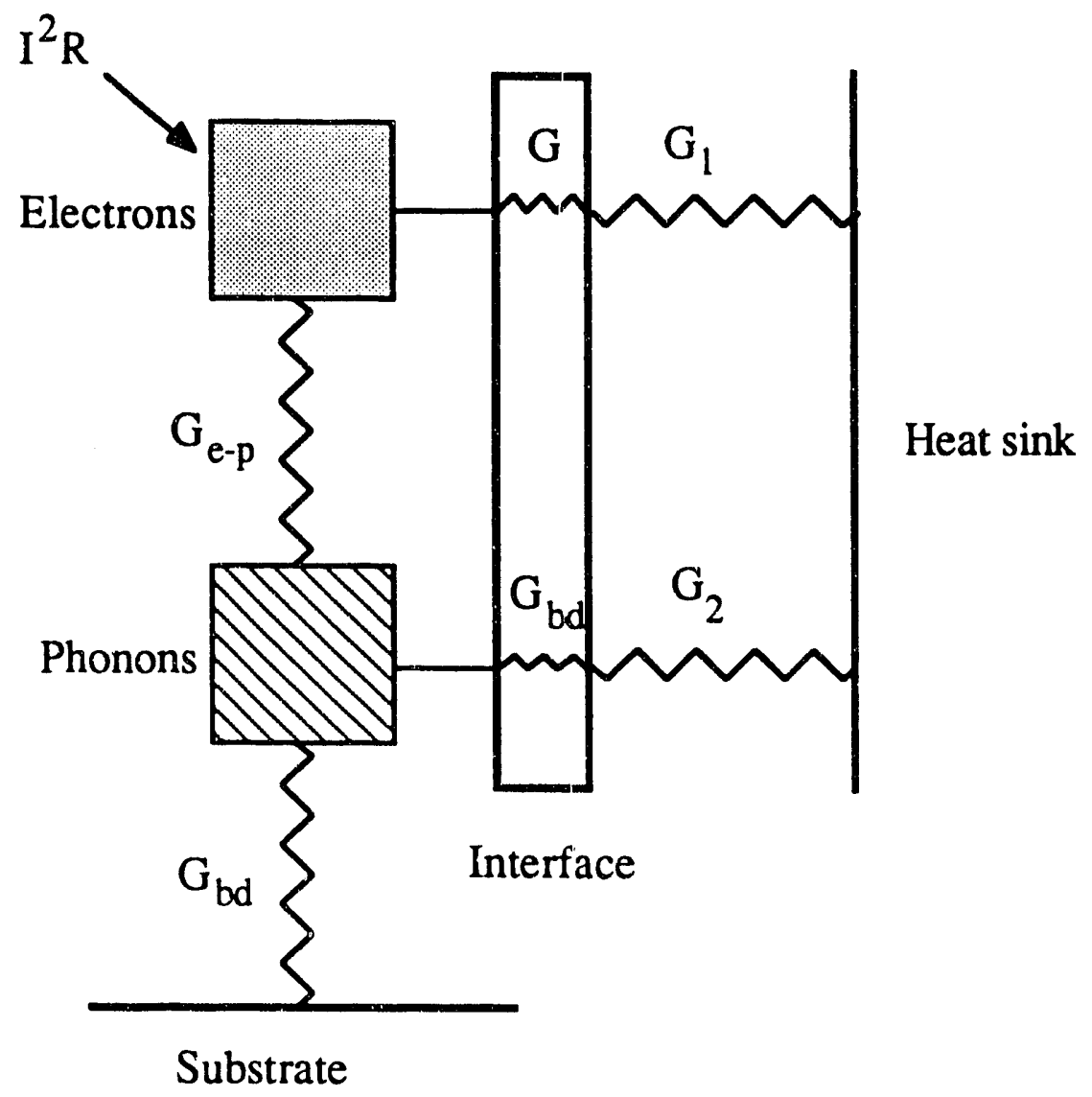

Fig. \%.3 Thermal circuit for the proposed experiment. 
conductance for electrons $G$, two requirements must be satisfied. First, the thermal conductance through the superconducting contact to the heat sink $G_{1}$ should be much larger than G. Otherwise, only the phonons would dissipate the absorbed energy. For the same reason, the thermal conductance due to phonons $G_{b d}$ should be much smaller than $G$. The conductances $G_{b d}$ and $G_{1}$ thus determine the minimum and maximum values of $G$ that can be measured.

To summarize, the following conditions must be satisfied in order for the experiment to succeed:

a) The superconducting film should be thinner than its penetration depth in order to be able to continuously vary its energy gap. For most films, a thickness of $\approx 750 \AA$ is sufficient. 110

b) The transition edge thermometer should function throughout the range of applied magnetic fields. This is accomplished by using a relatively thin thermometer strip, so as to increase its critical field. In the following section I will present data on the magnetic field dependence of the resistive transition of aluminum films.

c) The thermal conductance through the superconducting contact $G_{1}$, should be made as large as possible. The thermal conductivity for normal electrons in the superconductor can be approximated from the Weiderman-Franz law $\kappa=2.5 \times 10^{8} \mathrm{~T}^{-1} \mathrm{Wcm}^{-1} \mathrm{~K}^{-1}$, where $\rho$ is the normal state electrical resistivity in $\Omega-\mathrm{cm}^{49}$ The thermal conductance is thus maximized by using a superconductor which has a low normal-state resistivity.

e) The thermal conductance for electrons through the thermometer strip $G_{s}$ should be larger than the thermal boundary conductance between the strip and the substrate. Otherwise, only phonons would contribute to the conduction process. This necessitates the use of a low resistivity metal for the transition edge thermometer..

d) It is necessary to minimize the phonon contribution to the energy dissipation process. This is achieved by minimizing the contact area of the thermometer strip with the substrate and the superconducting contacts. 
There are relatively few material choices that can satisfy the above mentioned requirements. A near optimum choice is an aluminum transition edge thermometer and lead superconducting contacts. Our calculations indicate that for these materials, and for the experimental configuration of Fig. 7.2, we expect to observe approximately three orders of magnitude increase in the measured thermal conductance as a function of applied magnetic field. At zero field, the measured conductance is primarily due to the phonon conduction $G_{b d}$, while at the critical field, the measured conductance is limited by $G_{1}$.

\subsection{Preliminary Results and Conclusions}

Our first measurements were meant to test the characteristics of aluminum transitionedge thermometers in an applied magnetic field. A home made superconducting magnet was constructed. Its inner diameter was $12 \mathrm{~mm}$, and it produced a field strength of 0.1 T/A. In addition, a temperature regulated stage was constructed to fit inside the magnet bore. The sample consisted of $500 \AA$ thick, $2 \mu \mathrm{m}$ wide, and $10 \mu \mathrm{m}$ long aluminum strip. Contacts to the edges of the strip were made with $\mathrm{Nb}$. The resistive transition for zero applied magnetic field, and for a parallel field of $0.2 \mathrm{~T}$ is shown in Fig. 7.4. The transition in the latter case is broad, but still useful. The thermal conductance from the strip to its environment was deduced from the measured I-V curves. The ac method (section 2.3) was used in order to avoid non-thermal non-linearities. The measured thermal conductance of $\approx 2.5 \times 10^{-8} \mathrm{~W} / \mathrm{K}$, was independent of the magnetic field. The prediction of the acoustic mismatch model for this configuration is $\approx 2 \times 10^{-8} \mathrm{~W} / \mathrm{K}$, indicating that the thermal conductance was primarily due to phonon transport. We did not expect to observe a change in the thermal conductance for this configuration for two reasons. First, the critical field for the niobium films is higher than that available with our apparatus, ${ }^{107}$ and hence the electrons in the aluminum are blocked from crossing the interface with the superconductor. Secondly, the electrical resistivity of niobium is high $(\approx 10 \mu \Omega-\mathrm{cm}),{ }^{69}$ thus significantly reducing the thermal conductance $G_{1}$ of the superconducting pads. 


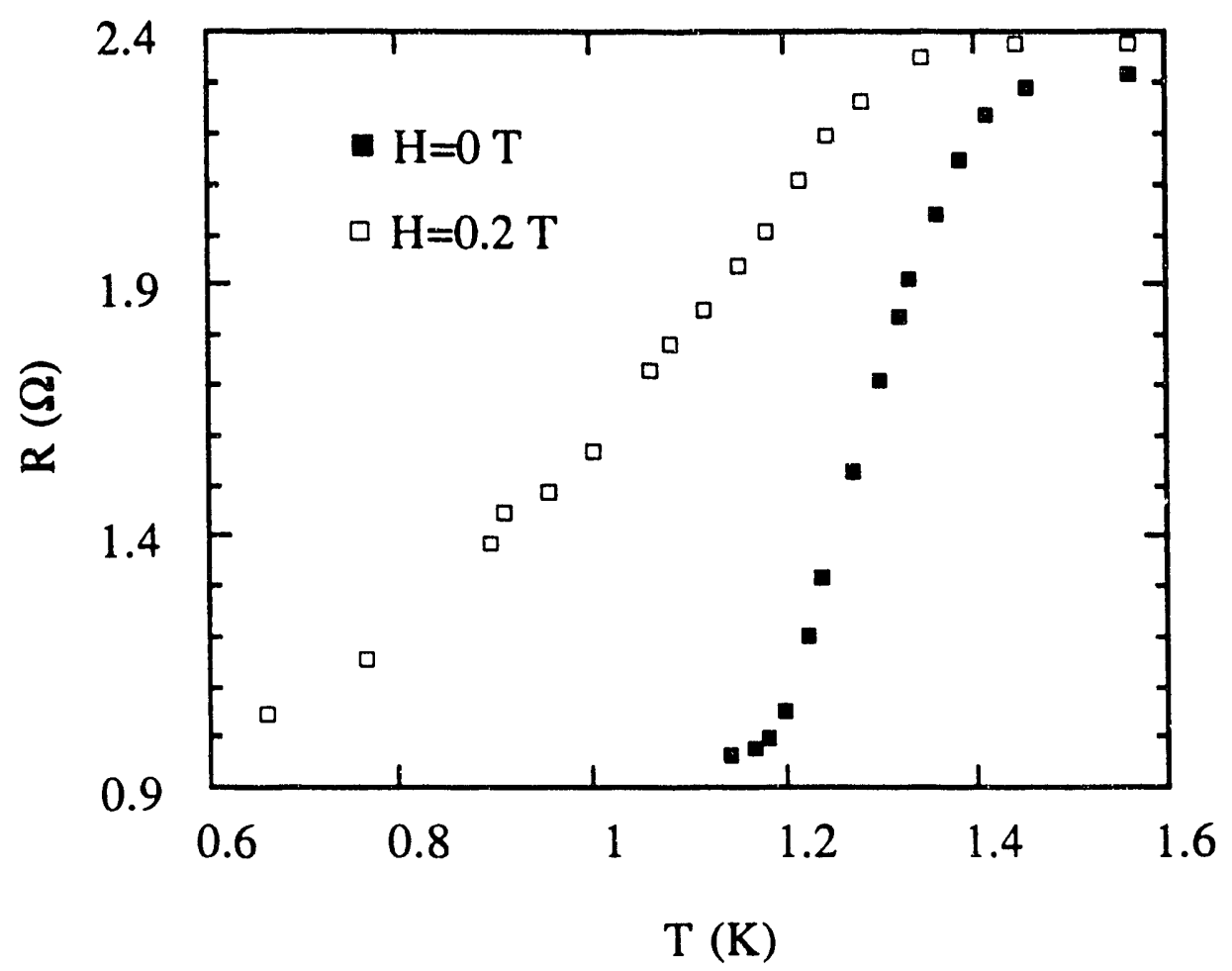

Fig. 7.4 Resistive transition for an aluminum strip for zero applied magnetic field, and a field of $0.2 \mathrm{~T}$, applied parallel to the film surface. The length of the strip is $10 \mu \mathrm{m}$, its width is $2 \mu \mathrm{m}$. The film is $400 \AA$ thick. Contact to the strip were made with $\mathrm{Nb}$ pads. 
Approximately two weeks prior to the writing of this dissertation, we received the first set of devices from Yale University that consisted of Al thermometers, Sn superconducting pads, and $\mathrm{Cu}$ heat sinks. Unfortunately, the aluminum strips did not make metalurgical contact to the tin pads. We plan to fabricate and measure additional devices in the near future. 


\section{REEERENCES}

1. J. G. Bednorz and K. A. Müller, Z. Phys. B 64, 189 (1986).

2. J. Clarke, Proc. IEEE, 77, 1208 (1989).

3. P. L. Richards, J. Clarke, R. Leoni P. H. Lerch, S. Verghese, M. R. Beasley,T. H. Gebaile, R. H.Hammond, P. Rosenthal, and S. R. Spielman, Appl. Phys. Lett. 54, 283 (1989).

4. Q. Hu and P. L. Richards, Appl. Phys. Lett. 55, 2444 (1989).

5. M. Nahum, Q. Hu, P. L. Sachtjen, N. Newman, and B. F. Cole, IEEE Trans. Magn. MAG-27, 3081 (1991).

6. E. Zeldov, N. M. Amer, G. Koren, and A. Gupta, Phys. Rev. B 39, 9712 (1989).

7. A. Frenkel, M. A. Saifi, T. Venkatesan, P. England, X. D. Wu, and A. Inam, J. Appl. Phys. 67, 3054 (1990).

8. M. Leung, P. R. Broussard, J. H. Claassen, M. Osofski, S. A. Wolf, and U. Strom, Appl. Phys. Lett. 51, 2046 (1987).

9. W. R. Donaldson, A. M. Kadin, P. H. Ballentine, and R. Sobolewski, Appl. Phys. Lett. 54, 2470 (1989).

10. M. Nahum, S. Verghese, P. L. Richards, and K. Char, Appl. Phys. Lett. 59, 2034 (1991).

11. M. Nahum and P. L. Richards, IEEE Trans. Magn. MAG-27, 2484 (1991).

12. J. Mees, M. Nahum, and P. L. Richards, Appl. Phys. Lett. 59, 2329 (1991).

13. M. Nahum, C. A. Mears, and P. L. Richards, IEEE Trans. Magn. (in press).

14. M. Nahum, C. A. Mears, and P. L. Richards, Proceedings of the Seventh International Conference on Phonon scattering in Condensed Matter, Comell University, Ithaca 1992, (in press).

15. A. F. Andreev, Sov. Phys. JETP 19, 1228 (1964).

16. T. W. Kenny, P. L. Richards, I. S. Park, E. E. Haller, and J. W. Beeman, Phys. 
Rev. B 39, 8476 (1989-II).

17. S. M. Grannan, A. E. Lange, E. E. Haller, and J. W. Beeman, Phys Rev B 45, 4516 (1991).

17a. N. Wang, F. C. Wellstood, B. sadoulet, E. E. Haller, and J. Beemen, Phys. Rev. B 41, 3761 (1990).

18. J. M. Lamar, Appl. Opt. 25, 870 (1986).

19. C. Kittel and H. Kroemer, Thermal Physics (Freeman, New-York, 1980).

20. R. A. Smith, F. E. Jones, and R. P. Chasmar, The Detection and Measurement of Infrared Radiation, 2nd ed. (Oxford U.P., Oxford 1968).

21. J. B. Johnson, Phys. Rev. 32, 97 (1928).

22. A. E. Lange, E. Kreysa, S. E. McBride, and P. L. Richards, Int. J. Infrared Millimeter Waves 4, 689 (1983).

23. P. M. Downey, A. D. Jeffries, S. S. Meyer, R. Weiss, F. J. Bachner, J. P. Donnelly, W. T. Lindley, R. W. Mountain, and D. J. Silversmith, Applied Optics 23, 910 (1984).

24. D. B. Rutledge, D. P. Neikirk, and D. P. Kasilingam, in Infrared and Millimeter Waves, edited by K. J. Button (Academic, New York, 1983), pp. 1-90.

25. J. Lesurf, Millimetre-Wave Optics, Devices \& Systems, (Adam Hilger, Bristol, 1990).

26. H. G. Booker, J. IEEE IIIA, 620 (1946).

27. T. L. Hwang, S. E. Schwarz, and D. B. Rutledge, Appl. Phys. Lett. 34, 773 (1979).

28. D. Miller, P. L. Richards, S. Etemad, A. Inam, T. Venkatesan, B. Dutta, X. D. Wu, C. B. Eom, T. H. Geballe, N. Newman, and B. F. Cole, Appl. Phys. Lett. 59, 2326 (1991).

29. R. E. Matick, Transmission Lines for Digital and Communication Networks (Mc Graw-Hill, New York, 1969). 
30. F. E. Ternman, Radio Engineers Handbook (Mc Graw-Hill, New York, 1943), p. 51.

31. Li Xizhi, P. L. Richards, and F. L. Loyd, Int. J Infrared Millimeter Waves 9, 101 (1988).

32. D. D. Dyson, I. R. E. Trans. Ant. Prop. AP.7, 181 (1959).

33. Y. S. Touloukian, ed., Thermophysical Properties of Matter (IFI/Plenum, New York, (1970), Vol. 2.

34. Y. S. Touloukian, ed., Thermophysical Properties of Matter (IFI/Plenum, New York, (1970), Vol. 5.

35. American Institute of Physics Handbook, 3rd ed. (McGraw-Hill, New-York, 1972).

36. N. Newman, K. Char, S. M. Garrison, R. W. Barton, R. C. Taber, C. B. Eom, T. H. Geballe, and B. Wilkens, Appl. Phys. Lett. 57, 520 (1990).

37. Apiezon Products Ltd., 8 York Road, London, SE1, U.K.

38. Lake Shore Cryotonics, Inc., 64 E. Walnut St. Westerville, OH 43081.

39. Ithaco Inc., 735 W. Clinton Street, Box 6437, Ithaca, NY 14851.

40. Emerson and Cuming, Inc., Canton, MA 02021.

41. M. Nahum and P. L. Richards, (unpublished).

42. G. M. Reibeiz, W. G. Regehr, D. B. Rutledge, R. L. Savage, C. W. Domier, and N. C. Luhmann Jr., Proceedings of the 11th International Conference on Infrared and Millimeter Waves, Pisa, Italy, 20-24 October 1986, p. 1.

43. G. L. Carr, M. Quijada, D. B. Tanner, C. J. Hirschmugl, G. P. Williams, S. Etemar, B. Dutta, F. DeRosa, A. Inam, T. Venkatesan, and X. Xi, Appl. Phys. Lett. 57, 2725 (1990).

44. W. S. Brocklesby, Don Monroe, A. F. J. Levi, M. Hong, S. H. Liou, J. Kwo, C. E. Rice, P. M. Mankiewich, and R. E. Howard, Appl. Phys. Lett. 54, 1175 (1989).

45. M. G. Forrester, M. Gottlieb, J. R. Gavaler, and A. I. Braginski, Appl. Phys. Lett. 
53, 1332 (1988).

46. M. I. Flick, P. E. Phelan, and C. L. Tien, Cryogenics 30, 1118 (1990).

47. W. A. Little, Can. J. Phys. 37, 334 (1959).

48. Tom Klitsner and R. O. Pohl, Phys. Rev. B 36, 6551 (1987).

49. C. Kittel, Introduction to Solid State Physics, 6th ed. (Wiley, New-York, 1988).

50. E. T. Swartz and R. O. Pohl, Rev. Mod. Phys. 61, 605 (1989).

51. E. T. Swartz and R. O. Pohl, Appl. Phys. Lett. 51, 2200 (1987).

52. E. T. Swartz, Ph. D. thesis, Cornell University (University Microfilms International, Ann Arbor, MI, 1987).

53. K. Char, N. Newman, S. M. Garrison, R. W. Barton, R. C. Taber, S. S. Laderman, and R. D. Jacowitz, Appl. Phys. Lett. 57, 409 (1990).

54. K. E. Goodson, (private communication).

55. S. J. Hagen, Phys. Rev. B 40, 9389 (1989).

56. K. Char (private communication).

57. C. Uher and A. B. Kaiser, Phys. Rev. B 36, 5680 (1987).

58. C. D. Marshall, I. M. Fishman, and M. D. Fayer, Phys. Rev. B 43, 2696 (1991).

59. S. G. Han, Z. V. Vardeny, K. S. Wong, O. G. Symko, and G. Koren, Phys. Rev. Lett. 65, 2708 (1990).

60. N. Bluzer, D. K. Fork, T. H. Geba!', M. R. Beasley, M. Y. Reizer, S. R. Greenfield, J. J. Stankus, and M. Fayer, IEEE Trans. Magn. MAG-27, 1519 (1991).

61. M. L. Roukes, M. R. Freeman, R.S. Germain, R. C. Richardson, and M. B. Ketchen, Phys. Rev. Lett. 55, 422 (1985).

62. P. L. Richards, Proceedings of the International School of Space Sciences, Summer School, L'Aquila. Italy, 1991.(to be published).

63. A. C. Clapp, C. Hagmann, S. Tanake, and P. L. Richards, (unpublished).

64. R. L. Kautz, J. Appl. Phys. 49, 308 (1978). 
65. L. Duband, D. Alsop, and A. Lange, Advances in Cryogenic Engineering, (Plenum Press, New York, 1990).

66. RV-Elektroniikka OY, Veraniehentie 14, SF 01510, Vantaa, Finland.

67. Muliti-Scanning Devices Corporation, 1 Fairchild Court, Plainview, NY 11803.

68. M. H. Read and C. Altman, Appl. Phys. Lett. 7, 51 (1965).

69. D. W. Face, Ph.D. thesis, Yale University, (Univ. Microfilms Intl., Ann Arbor, MI.) 1988 .

70. D. E. Prober (private communication).

71 D. C. Alsop, C. Inman, A. E. Lange, and T. Wilbanks, Appl. Opt., (in press).

72. P. H. Siegel, IEEE Microwave Theory Tech. Dig. MTT-S, 649 (1986).

73. P. P. Tong, Ph. D dissertation, California Institute of Technology, 1984.

74. H. Ekstrom, S. Gearhart, P. R. Acharya, H. Dave, G. Rebeiz, S. Jacobson, E. Kollberg, and G. Chin, in Third International Conference on Space Terahertz Technology, Ann Arbor, Michigan, March 1992, pp. 280-290.

75. D. R Dykaar, B. I. Greene, J. F. Federici, A. F. Levi, L. N. Pfeiffer, and R. F. Kopf, Appl. Phys. Lett. 59, 262 (1991).

76. R. H. DuHamel and D. E. Isbell, IRE National Convention Record I, 119 (1957).

77. Princeton Applied Research, P.O. Box 2565, Princeton, NJ 08543.

78. J. Clarke, G. I. Hoffer, P. L. Richards, and N. H. Yeh, J. Appl. Phys. 48, 4865 (1977).

79. E. N. Grossman, J. E. Savageau, and D. G. McDonald, Appl. Phys. Lett. 59, 3225 (1991).

80. G. W. Chantry, Long-Wave Optics, (Academic Press, London, 1984).

81. J. B. Peterson and P. L. Richards, Int. J. Infrared Millimeter Waves 5, 1507 (1984).

82. R. Winston, J. Opt. Soc. Am. 60, 245 (1970).

83. W. H. Chang, J. Appl. Phys. 50, 8129 (1979). The values of $k$ are for a microstrip embedded in a dielectric. 
84. G. L. Matthaei, L. Young, and E. M. T. Jones, Design of Microwave Filters, Impedance-Matching Networks, and Coupling Structures (Mc Graw-Hill, New York, 1964).

85. E. G. Cristal, IEEE Trans. Microwave Theory Tech. MTT-19, 486 (1971).

86. A. B. Smith, J. A. Carpenter, and L. Lee, Bull. Am. Phys. Soc. 35, 378 (199i).

87. J. C. Nabity and M. N. Wybourne, Phys. Rev. B 44, 8990 (1991-II).

88. F. C. Wellstood, Ph.D. thesis, University of California at Berkeley, (Univ. Microfilms Intl., Ann Arbor, MI.) 1988, and references therein.

89. M. L. Roukes, Ph.D. thesis, Cornell University, (Univ. Microfilms Intl., Ann Arbor, MI.) 1985, and references therein.

90. A. C. Anderson and R. E. Peterson, Phys. Lett. 38A, 519 (1972).

91. F. C. Wellstood, C. Urbina, and J. Clarke, Appl. Phys. Lett. 54, 2599 (1989).

92. L. Solymar, Superconductive Tunnelling and Applications, (Wiley, New York, 1972).

93. D. Rogovin and D. J. Scalapino, Ann. Phys. 86, 1-90 (1974).

94. H. Pothier, Ph.D. thesis, Docteur de l'Universite Paris 6, 1991 (unpublished).

95. D. J. Goldie, N. E. Booth, C. Patel, and G. L. Salmon, Phys. Rev. Lett. 64, 954 (1990).

96. H. Kraus, F. von Feilitzsch, J. Jochum, R. L. Mössbauer, Th. Peterreins, and F. Pröbst, Phys. Lett. B 231, 195 (1989)

97. C. A. Mears, S. E. Labov, G. W. Morris, C. E. Cunningham, M. A. LeGros, E. H. Silver, A. T. Baefkntcht, N. W. Madden, D. A. Landis, F. S. Goulding, R. W. Bland, K. E. Laws, and R. C. Dynes, IEEE Trans. Magn., (in press).

98. A. B. Pippard, F. R. S., J. G. Shephers, and D. A. Tindall, Proc. Roy. Soc. Lond. A. 324,17 (1971)

99. J. R. Waldam, Proc. R. Soc. Lond. A. 345, 231 (1975).

100. I. L. Landau, JETP Lett. 11, 437 (1970). 
101. T. Y. Hsiang, Ph.D. thesis, University of California at Berkeley, (Univ. Microfilms Intl., Ann Arbor, MI.) 1977.

102. R. Kümmel, U. Gunsenheimer, and R. Nicolsky, Phys, Rev. B 42, 3992 (1990).

103. G. E. Blonder, M. Tinkham, and T. M. Klapwijk, Phys. Rev. Lett. B 25, 4515 (1982).

104. M. Octavio, M. Tikham, G. E. Blonder, and T. M. Klapwijk, Phys. Rev. B 27, 6739 (1983).

105. T. M. Klapwijk, G. E. Blonder, and M. Tinkham, Physica 109 \& 110 B, 1657 (1982).

106. L. P. Gor'kov, JETP 36, 1918 (1959).

107. T. V. Duzer, Principles of Superconductive Devices and Circuits, (Elsevier, New York 1981).

108. D. H. Douglass, Jr., Phys. Rev. Lett. 7, 14 (1961).

109. V. L. Ginzburg and L. D. Landau, JETP 20, 1064 (1950).

110. D. H. Douglass, Jr. and R. Meservey, Proceedings of the Eighth International Conference on Low Temperature Physics, (Butterworth Scientific Publications Ltd., London, 1963).

111. D. E. Morris and M. Tinkham, Phys. Rev. Lett. 6, 600 (1961).

112. M. Tinkham, IBM Journal, 49 (1962).

113. Infrared Laboratories Inc., 1808 E. 17th St., Tucson, AZ 85719.

114. R. A. Serway, Physics: For Scientists and Engineers, (CBS College Publishing, New York, 1982).

115. D. E. Prober, Rev. Sci. Instrum. 45, 849 (1974).

116. Stanford Resarch Systems Inc., 1290 D Reamwond Av., Sunnyvale, CA 94089.

117. Krohn-Hite Corporation, 580 Massachusetts Av., Camuridge, MA 02139.

118. Hewlett Packard Inc., P. O. Box 301, Loveland, CO 80537.

119. C. A. Mears (private communication). 
120. Tamura Corporation of America, 1150 Dominguez St., Carson, CA 90746.

121. Triad Ultrad, 1124 E. Franklin St., Huntington, IN 46750. 


\section{Appendix A: Transformers as Amplifiers for Low Impedance Devices}

All of the detector concepts that are described in this dissertation, as well as many other superconducting devices have typical impedances in the range of 1-100 $\Omega$. In some cases, the useful sensitivity of these devices can be limited by the noise introduced at the first stage of amplification. The noise of an ideal amplifier should always be lower than the Johnson noise of the source resistance, since this is the minimum noise across any resistive device. A useful figure of merit for an amplifier is its noise temperature $T_{n}$. It is the temperature of the source resistor $R_{1}$, whose Johnson voltage noise $\left(4 k T_{n} R_{1}\right)^{1 / 2}$, equals the amplifier noise. The noise temperature of the amplifier depends on the source resistance, and is considered to be unimportant when $T_{n}$ is lower than the operating temperature of the device. The best available FET amplifiers typically have noise temperatures of $\approx 0.1 \mathrm{~K}$, but this performance is only available for source impedances $R_{1} \geq 1$ $M \Omega .^{113} \mathrm{~A}$ convenient solution to the impedance mismatch between the low source impedance of superconducting devices and the optimum impedance for a conventional low noise amplifier, is to use a transformer before the first stage of amplification. The impedance seen at the output of an ideal transformer, with a turn ratio $n=N_{2} / N_{1}$, whose input is connected to a source resistance $R_{1}$, is given by $n^{2} R_{1} \cdot{ }^{114}$ Here $N_{1}$ and $N_{2}$ are the number of primary and secondary windings respectively. It is for this reason that a transformer is a used with voltage sources with low source impedances. Transformers operated at room temperature often contribute significant noise when used with cooled sources, this noise is primarily due to the Johnson noise in the resistance of the transformer windings. The solution to this problem is to cool the transformer. ${ }^{115}$ The Johnson noise will then be reduced because of the lower temperature, as well as the lower winding resistances available. Although the implementation of these concepts appears to be straightforward, the performance of a real transformer is usually far from ideal, and the differences can often complicate the measurement. In what follows, I will describe the 
basic operating principles of a transformer, and the factors that influence its useful characteristics. I will then discuss the practical details associated with the implementation and use of transformers at both LHe and room temperatures, and present data on a few useful transformers.

The figures of merit for a transformer are its voltage gain $\mathrm{G}$ and frequency bandwidth. The circuit in Fig. A.1 represents a transformer having no intrinsic losses, and is useful for analyzing its low frequency properties. The source is represented by a resistance $R_{1}$ in series with a voltage source $V_{1}$. The blocking capacitor $C_{1}$ prevents dc current from flowing through the transformer windings, a feature that is important when measuring the signal across a device that is biased with a constant current (see Fig. 3.4 for a useful example). The primary winding inductance is $\mathrm{L}_{1}$, the secondary inductance is $\mathrm{L}_{2}$, and the mutual inductance is $M$. The output of the transformer is shunted by a resistance $R_{2}$, and the output voltage $V_{2}$ is measured across the shunt resistance. Usually $R_{2}$ is the input impedance of the amplifier that follows the transformer. The gain of the transformer is then defined as the ratio $\mathrm{G}=\mathrm{V}_{2} / \mathrm{V}_{1}$. Using simple circuit analysis techniques it can be shown that for a frequency $\omega=2 \pi f$,

$$
G=\left[\left(\frac{R_{1}}{\omega M}+\frac{L_{2}}{\omega C_{1} M R_{2}}\right)^{2}+\left(\frac{1}{\omega^{2} C_{1} M}-\frac{R_{1} L_{2}}{R_{2} M}-\frac{L_{1}}{M}\right)^{2}\right]^{-1 / 2}
$$

Important conclusions in the gain equation include:

a) In order that the transformer gain not degrade, the shunt resistance $R_{2}$ should be much larger than the product of the source resistance times the ratio of the secondary to primary impedances $R_{1} L_{2} / L_{1}$. In practice, an amplifier that has an input impedance $\geq 10^{8} \Omega$ is sufficient.

b) The primary inductance $L_{1}$ limits the gain for frequencies $\omega<R_{1} / L_{1}$ even when $C_{1}$ and $\mathbf{R}_{\mathbf{2}}$ are large. 


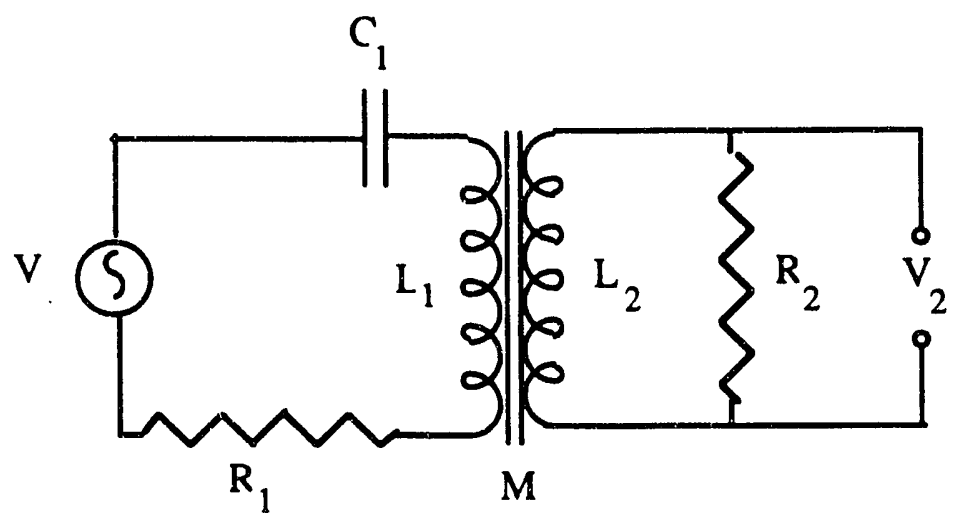

Fig. A.1 Electrical circuit of an ideal transformer that can be used to model its low frequency characteristics. 
c) The blocking capacitor can also influence the gain for frequencies $\omega<R_{1} / C_{1}$. For the rolloff associated with the capacitance to occur at a lower frequency than that associated with the primary inductance, it is necessary to pick $\mathrm{C}_{1}>\mathrm{L}_{1} / \mathrm{R}_{1}^{2}$.

d) A resonance in the gain curve occurs when $\omega=\left(\mathrm{L}_{1} \mathrm{C}_{1}\right)^{-1 / 2}$. This effect can provide useful additional gain when the source resistance $R_{1}$ is not too high.

The simple model presented above does not include the effects of leakage reactance and distributed capacitance in the windings, which reduce the transformer gain at high frequencies. Unfortunately, as the number of windings is increased, both the leakage reactance and the winding capacitance increase. This effect can be minimized by employing transformer core material of very high permeability, so as to increase the relevant inductances without increasing the number of windings. For most applications involving bolometric detectors, where the relevant frequencies are typically $\leq 1 \mathrm{kHz}$, the simple analysis presented here provides a useful and intuitive understanding of the transformer characteristics.

In order that the noise temperature of the transformer be lower than the temperature of the source resistance, two conditions have to be satisfied:

a) The Johnson noise from the resistance of the transformer windings should be lower than the Johnson noise of the device to be measured. For the low source impedances of interest here, this inevitably means that the transformer has to be cooled to LHe temperatures. Typical winding resistances at $300 \mathrm{~K}$ are several ohms, while at LHe temperatures they can be lower than $0.1 \Omega$.

b) The transformer will be useful only if it can match between the device impedance of several ohms, and the optimum impedance of $\approx 1 \mathrm{M} \Omega$ for conventional low noise amplifiers. This means that the transformer gain should be $10-100$.

Unfortunately, transformers with the above specifications are difficult to find. In 1974, D. E. Prober discussed the electrical performance of a Triad G-4 transformer that was cooled to liquid helium temperatures. ${ }^{115} \mathrm{He}$ was able to observe the Johnson noise in 
a $1 \Omega$ resistor at $4.2 \mathrm{~K}$, which translates into a voltage noise sensitivity of $\approx 10^{-11} \mathrm{VHz}^{-1 / 2}$ for a narrow band centered at $500 \mathrm{~Hz}$. The performance of this rather large transformer was far from ideal for source impedances larger than $\approx 20 \Omega$. Furthermore, I found that the gain of this transformer was significantly degraded when used in conjunction with a blocking capacitor. The reason is probably due to the large primary inductance (the transformer is physically large and thus has many windings), which implies that even moderately high capacitance values will degrade the transformer gain.

The most useful transformer for our purposes was produced by the United Transformer Corporation, about 20 years ago. The model O-14 transformer was initially embedded in wax which was subsequently removed by warming the transformer on a hotplate. It was necessary to remove the wax in order to allow operation at LHe temperatures. ${ }^{115}$ The transformer was then mounted in a lead shielded box $\left(\approx 1.5^{\prime \prime}\right.$ on a side) and was tested both at room temperature and pumped LHe temperature. A blocking capacitor $\mathrm{C}_{1}=10 \mu \mathrm{F}$, was also placed inside the box. The gain characteristics of the transformer, shown in Fig. A.2, were measured using the white noise technique with a HP model $3582 \mathrm{~A}$ spectrum analyzer. The measured gain had a value of $\approx 50$ and was independent of source resistance up to $\approx 100 \Omega$. The resonance peak however, was damped for higher source impedances. The $200 \mathrm{~Hz}$ shift in the resonant frequency upon cooling, indicates that the primary inductance was reduced by approximately a factor of 5 . This is probably due to a reduction of the magnetic permeabilty of the transformer core material. The primary inductance can be estimated from the frequency at which the resonance in the gain occurs. For the $300 \mathrm{~K}$ case, I calculate $\mathrm{L}_{1}=\omega^{-2} \mathrm{C}_{1}{ }^{-1}=0.09 \mathrm{H}$. The secondary inductance $L_{2}$ can be estimated by multiplying the primary inductance by $n^{2}$, where $\mathrm{n}$ is the known turn ratio. The mutual inductance is calculated from the value of the measured gain at frequencies where the gain is weakly dependent on frequency. At these frequencies Eq. A.1 predicts $G=M / L_{1}$. The predicted frequency dependence of the transformer gain at $300 \mathrm{~K}$, using no adjustable parameters is shown in Fig. A.2. The fit is 


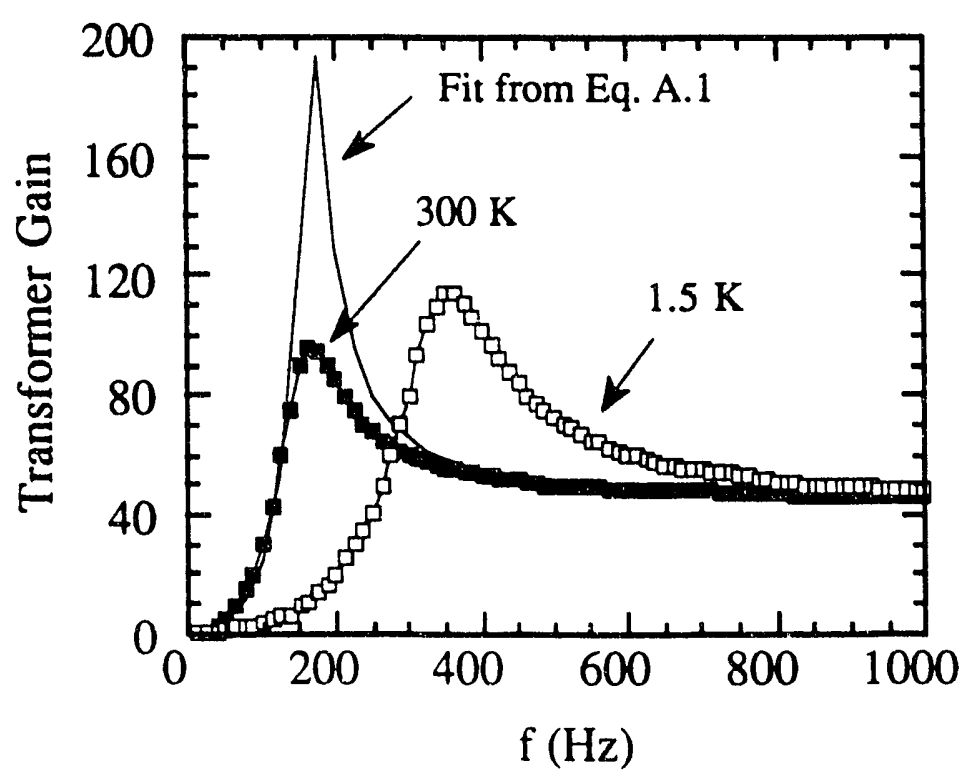

Fig. A.2 Gain versus frequency characteristics for a UTC Model O-14 transformer, used in conjunction with a $10 \mu \mathrm{F}$ blocking capacitor and a $15 \Omega$ source resistance. The gain was measured at $300 \mathrm{~K}$, and at $1.5 \mathrm{~K}$. The fit of Eq. A. 1 to the $300 \mathrm{~K}$ data is also shown. 
quite good for all frequencies except at the resonance peak, where the predicted gain is a factor of $\approx 2$ higher than the measured value. This is not surprising, considering the fact that our model does not incorporate any loss mechanisms, which wiil inevitably reduce the quality factor $Q$ of the resonance. For most practical purposes however, the simplified model can be used to predict the transformer characieristics.

The noise contribution of the cooled transformer was measured by shunting its input with a $20 \Omega$ resistor that was maintained at $0.3 \mathrm{~K}$, and measuring the resultant voltage noise. The output of the transformer was connected to a Stanford Research Systems model SR560 amplifier (gain=104), ${ }^{6}$ followed by a Krohn-Hite model 3750 variable bandpass filter (gain=10). ${ }^{117}$ The amplified voltage noise was then measured by an H.P. model 3582A spectrum analyzer. ${ }^{118}$ The measured voltage noise when referred to the input of the transformer, was $7 \times 10^{-11} \mathrm{VHz}^{-1 / 2}$ at $1 \mathrm{kHz}$. The Johnson noise resulting from the source resistance shunting the input is only $2 \times 10^{-11} \mathrm{VHz}^{-1 / 2}$, implying an excess voltage noise of $6.7 \times 10^{-11} \mathrm{VHz}^{-1 / 2}$. This excess noise is mostly due to the input voltage noise of $3 \times 10^{-9} \mathrm{VHz}^{-1 / 2}$ from the SRS amplifier, which is equivalent to $6.5 \times 10^{-11} \mathrm{VHz}^{-1 / 2}$ when referred to the input of the transformer. The noise contribution of this second stage of amplification can be reduced by using high quality JFET amplifiers, which have a voltage noise as low as $\approx 5 \times 10^{-10} \mathrm{VHz}^{-1 / 2}$ referred to their input. ${ }^{119}$

The gain characteristics of other transformers was also measured. These included miniature transformers manufactured by Tamura Corporation, ${ }^{120}$ as well as a Triad model G-10 transformer. ${ }^{121}$ These transformers however, exhibited poor characteristics when used in corjunction with blocking capacitors up to $\mathrm{C}_{1}=1 \mathrm{mF}$, and cooled to $\mathrm{LHe}$ temperatures.

To summarize, it appears that cooled transformers can be used successfully with low noise amplifiers to produce noise temperatures below $1 \mathrm{~K}$ for source impedances between 1 and $100 \Omega$. In addition, the transformer characteristics at low frequencies can be analyzed in terms of a simple electrical model. On the other hand, it is difficult to find 
transformers that satisfy all of the requirements. Furthermore, the physical size of many useful transformers is rather large and is thus unsuitable for applications requiring array compatibility.

A different approach to the matching problem is to use a DC SQUID as a low noise amplifier. It is now possible to produce DC SQUIDs that occupy an area of $\approx 1 \times 1 \mathrm{~mm}^{2}$, and have noise temperatures $\leq 10^{-6} \mathrm{~K}$ for source resistances $\leq 200 \Omega{ }^{2}$ Furthermore, it is relatively simple to integrate the SQUIDs in an array format. The potential disadvantages of this technique are the relative complexity of operation, as well as the need to use a transformer at the output of the SQUID. 

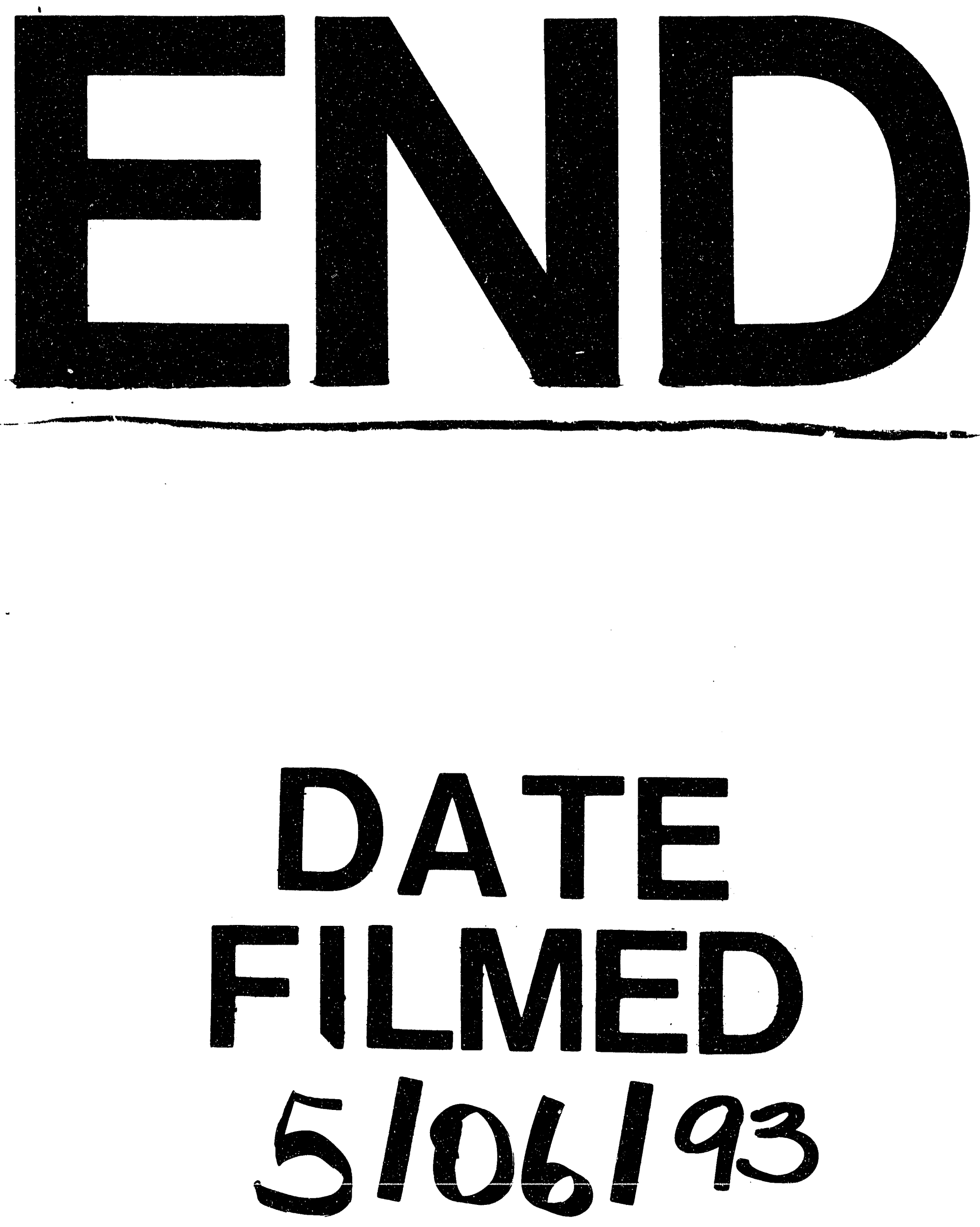
